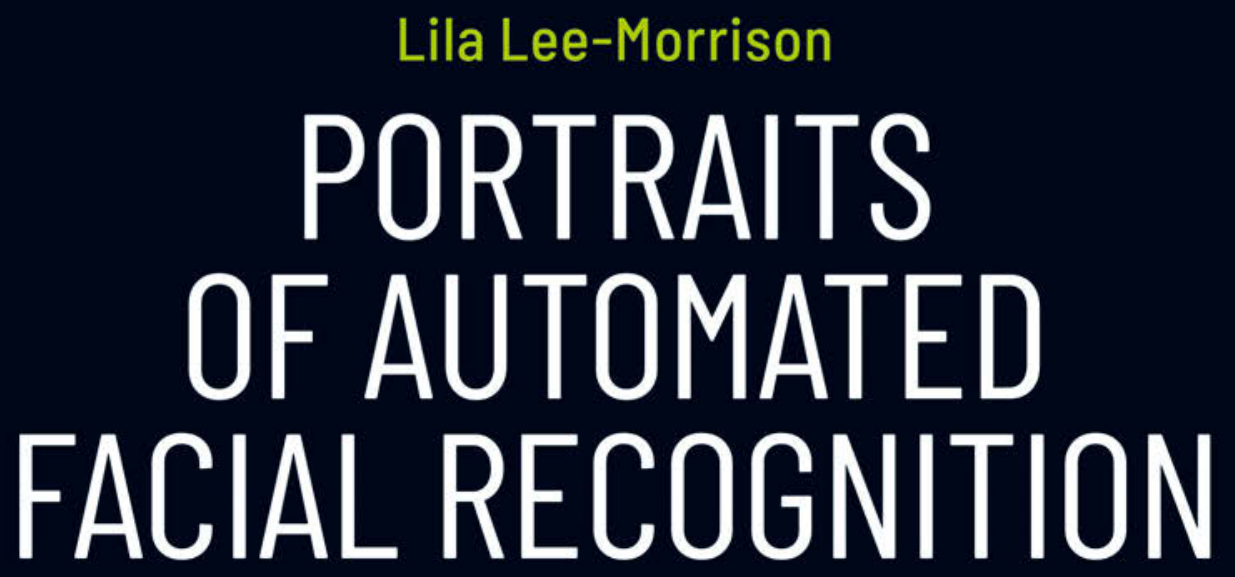

On Machinic Ways of Seeing the Face 
Lila Lee-Morrison

Portraits of Automated Facial Recognition

Image | Volume 162 
to the memory of my father, Jong Kwan Lee (1940-1988)

Lila Lee-Morrison, born in 1977, is a PhD student of Art History and Visual Studies at Lund University, Sweden. She has published texts on the political and cultural implications of machine vision, including the use of biometrics and drone warfare. She received a B.A. in Political Science from Hunter College, NY and an M.A. in Visual Culture from Lund University. 
Lila LeE-Morrison

\section{Portraits of Automated Facial Recognition}

On Machinic Ways of Seeing the Face

[transcript] 
The first section of this book is adapted from an abridged article titled, "A Portrait of Facial Recognition: Tracing a history of a statistical way of seeing" in Philosophy of Photography, Vol. 9:2, 2018.

This book is published through generous financial support from: Längmanska Cultural Foundation and Gyllenstiernska Krapperup Foundation

\section{Bibliographic information published by the Deutsche Nationalbibliothek}

The Deutsche Nationalbibliothek lists this publication in the Deutsche Nationalbibliografie; detailed bibliographic data are available in the Internet at http://dnb.d-nb.de

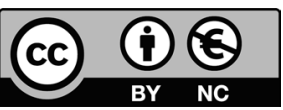

This work is licensed under the Creative Commons Attribution-Non Commercial 4.0 (BY-NC) license, which means that the text may be may be remixed, build upon and be distributed, provided credit is given to the author, but may not be used for commercial purposes. For details go to: http://creativecommons.org/licenses/by-nc/4.o/

Permission to use the text for commercial purposes can be obtained by contacting rights@transcript-verlag.de

Creative Commons license terms for re-use do not apply to any content (such as graphs, figures, photos, excerpts, etc.) not original to the Open Access publication and further permission may be required from the rights holder. The obligation to research and clear permission lies solely with the party re-using the material.

\section{(C) 2019 transcript Verlag, Bielefeld}

Cover layout: Maria Arndt, Bielefeld

Cover illustration: Wonju Lee, Minkyu Cheon, Chang-ho Hyun, and Mignon Park, "Eigenfaces: Mean Face", 2013. (C) Wonju Lee, Minkyu Cheon, Changho Hyun, and Mignon Park. Used under CC BY 3.0 (http://creativecommons .org/licenses/by/3.0) / Cropped from original.

Proofread by Tim Carter

Typeset by Justine Buri, Bielefeld

Printed by Majuskel Medienproduktion GmbH, Wetzlar

Print-ISBN 978-3-8376-4846-1

PDF-ISBN 978-3-8394-4846-5

https://doi.org/10.14361/9783839448465 


\section{Table of Contents}

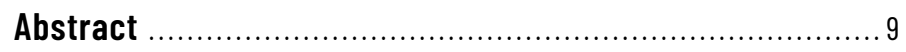

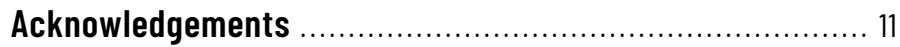

Chapter 1: Introduction ..................................................... 15

On Machinic Ways of Seeing the Face: Aim and Background ........... 15

Cultural Analyses of Biometrics: Previous Scholarship ................ 21

Language and Visual Artifacts: Empirical Material .................... 27

Temporalities - History as Critical Inquiry: Methodology .............. 33

Machinic Observer - Seeing as Recognition:

Theoretical Framework ................................................... 38

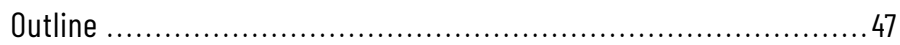

\section{Part I: Aesthetics of an Algorithm}

Chapter 2: Eigenface ….................................................. 55

Background: Eigenface in the Development of AFR Technology ....................................................... 55

Bledsoe: "The Model Method in Facial Recognition" ................... 57

Representational Mechanisms and the Machinic Observer ..............61 61

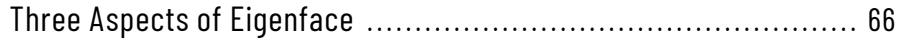

The Eigenface Image ….................................................... 78 
Chapter 3: Francis Galton and the Composite Portrait ...... 85

Pictorial Statistics ............................................................. 85

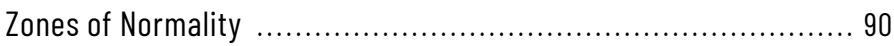

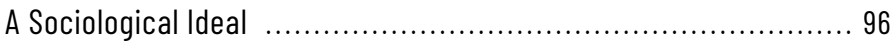

Chapter 4: Wittgenstein and the Composite Portrait ......... 101

A Destabilization of Vision ............................................... 102

Aspect Perception and Aspect Blindness ................................. 108

Negotiations of Recognition ................................................ 110

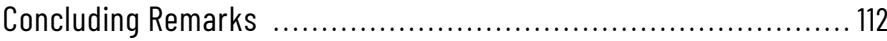

\section{Part II: Artistic Interventions}

Chapter 5: Portraiture in the Age of AFR ............................ 117

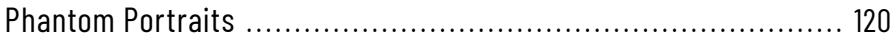

Chapter 6: Metaportraits: Thomas Ruff, andere Portraits .... 125

Background: The Composite Portrait in Contemporary Art ........... 125

Identification Portraiture .............................................. 126

andere Portraits (1994-1995) ............................................ 129

Metaportraits and the Binary Face ......................................... 131

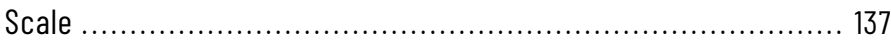

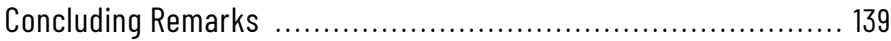

\section{Chapter 7: Faces in Excess:}

Zach Blas, Facial Weaponization Suite .............................. 141

Background: A Conceptual Framework .................................. 141

Facial Weaponization Suite (2011-2014) ................................. 145

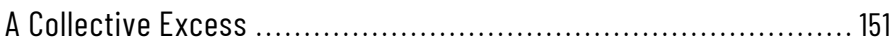

Concluding Remarks .................................................... 156

Chapter 8: An Algorithmic Ready-made:

Trevor Paglen, Adversarially Evolved Hallucination and Eigenface (Even The Dead Are Not Safe) ..................... 159

Background: Notions of transparency .................................... 159

Adversarially Evolved Hallucination (2017) ............................. 162 
Eigenface (Even the Dead Are Not Safe) (2017) 169

Concluding Remarks

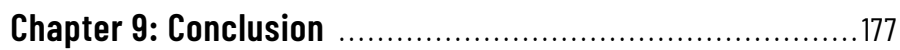

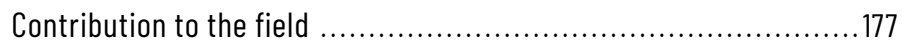

Revisiting the Aim: Looking Back ....................................... 180

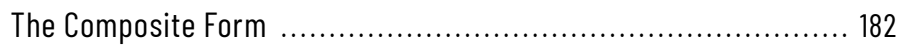

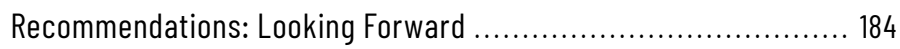

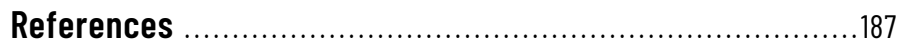

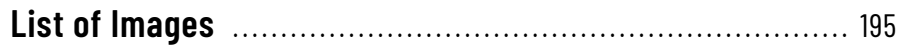





\section{Abstract}

This book offers a unique analysis of the use of the automated facial recognition algorithms that are increasingly intervening in our society, from a critical visual culture studies perspective. The first part of this study traces the history of the merging of statistics and vision, examining the example of an early facial recognition algorithm called "eigenface," while the second part addresses contemporary artistic engagements with facial recognition technology in the work of Thomas Ruff, Zach Blas and Trevor Paglen. This book argues that we must take a closer look at automated facial recognition and claims that this technology is embedded in historical practices of visuality. Even more significantly, this technology, the book argues, is redefining what it means to see and be seen in the contemporary world. 



\section{Acknowledgements}

Throughout the development of this book, I have been helped by a wide swathe of people, places and things which provided support and guidance. Firstly, I would like to thank my main supervisor Max Liljefors, who wouldn't describe it this way, but for supporting a Korean-American woman from New York in a predominantly homogeneous, Scandinavian academic environment at the department in Lund and in the wider academic field of Art History and Visual Studies in Sweden. During my position at the university I have been reminded several times of a level of radical open-mindedness he has had in his support of my research and I am eternally grateful. I am also indebted to him for his eloquent, humorous, clear and always hopeful guidance in discussions throughout the research and writing process. His sustaining belief in the value of my work, especially in moments when I didn't have it myself was a source of immense support in the long and at times, arduous process of writing.

Thank you to my colleagues in the Department of Arts and Cultural Sciences at Lund University, the Digital Cultures Research Node and especially to those within my division of Art History and Visual Studies. Special thanks to Peter Bengtsen for his generosity in sharing his practical knowledge and navigating the bureaucracy of academic and grant funding institutions, Erika Larsson for support, discussion and friendship and Ludwig Qvarnström for help in Swedish language translation. I would also like to thank the community of doctoral students in the wider department and a special thanks to Dr. Lisa Olsson Dahlquist for friendship and comradery during the final phases of writing. I am also thankful to my students enrolled in the Visual Culture Master's Program (2016-2018 
and 2018-2020) for the engaging discussions held in class and in seminars, covering much of the theoretical framework in this book.

I would like to acknowledge and thank the funding institutions of Dr. Uno Otterstedt's Fund and William Karlsson's Scholarship Fund at Lund University whose issuing of travel grants greatly benefitted this project.

Thanks to my co-supervisor Robert Willim for his inspired dialogue and feedback, Marta Braun for a supportive reading on an early, grammatically-challenging sketch of the eigenface chapter, Nanna Thylstrup and Daniela D'Agostinho for their helpful suggestions midway through the process and Liv Hausken for her informed reading and comments on a close to completed manuscript. A sincere thanks also goes to Tim Carter for a thorough and nuanced job of proofreading.

My research was greatly developed and bettered through discussions held with scholars at the conferences I participated in. I'd like to give acknowledgement to the participants of the "Precarious Aesthetics" conference held at UC Berkeley (2015), the "Uncertain Image" workshop at University of Copenhagen (2017) and the "Facial Machines" symposium held at the University of Aarhus, DK (2018). A special thanks also goes to the scholars of the Uncertain Archives research group at the University of Copenhagen, whose organization of pertinent events and invitations to participate throughout my research, deeply informed and pushed my work forward.

I'd like to acknowledge and give a special thanks to the artists included in this study for their intelligence and generosity: Zach Blas, Thomas Ruff and especially Trevor Paglen for fun discussions in Berlin. Thanks to the Film Archives of the Museum of Modern Art, New York for access to the video archives and textual materials of Harun Farocki and to Louise Wolthers at Hasselblad Foundation for guided access to the "Watched!" exhibit.

Thanks also to those who have aided in one way or another through discussions concerning the research, writing and the thinking process: Caroline Holmqvist, Henrik Lund Jørgensen, Johan Röing, Maria Norstedt, James Conant, Brooke Belisle, Anthony Paré, Arild Fetveit, Fred Ritchin, Tom Mitchell and Anders Michelsen. 
An enduring gratitude goes to my friends and family without whom I wouldn't be here: First and foremost, JC, my partner who truly makes it all possible and to my daughter Lulu, for whom the possibilities are endless. I am grateful to you both for bringing me down to earth and surrounding me with love. To my sister, Jennie Jieun Lee and Graham Collins, my oma, Hea Young Lee and stepfather, Peter Kim and to extended family Pete Chung and Patty Lu. I would also like to thank my friends who aided me in coming to Sweden in the first place, Jenny and Clay Ketter and Karin and Alex Hartley. Special thanks to Barbara Pollack for her initial encouragement to re-enter academia and for support commiserate in writing. Also, a heartfelt thanks goes to Anna Delarosa for always helping me see myself in the process.

Lastly, I'd also like to acknowledge a place: "Kallis" in Malmö for always giving me the depth of a framed horizon and an open sky to look at; offering clarity, sustenance and breathing space throughout. 



\section{Chapter 1: Introduction}

\section{On Machinic Ways of Seeing the Face: Aim and Background}

While passing through border control at Helsinki airport on a flight to Seoul, I walked through a biometric turnstile. On a small screen by the entrance to the turnstile were instructions to scan my passport. Once my passport had been scanned, I was then allowed to enter a small cubicle space between two panes of glass. I stood facing an empty screen that moved vertically, adjusting automatically to my height. A sensor took my picture, which then appeared before me on the screen. I waited a moment while the machine verified my identity by checking the facial image it had just captured against the image scanned from my passport. Then one of the glass panes opened, and I was released from the turnstile. I was then confronted with a border control guard - a man who sat behind a desk and whose face was shielded by a dark pane of glass. I only saw his hands, which grasped my documents. He did not seem to look at my face, but rather only examined my plane ticket and passport, which he eventually stamped with approval. My identity had been successfully recognized, and I was allowed to move on.

Automated facial recognition (AFR) has increasingly become a filter through which access to the world is granted. The ritual to which I was subjected at Helsinki airport, a ritual I also experienced in a slightly altered form at the Swedish immigration office, as part of the process of registering for permanent residency, is a familiar one. Beyond its most conspicuous uses in border control and immigration services, AFR is now increasingly being implemented in more mundane and everyday scenarios. For example, AFR systems 
are used in our phones, at ATM machines, in office security systems, for the manning of cash registers in convenience stores and inside toilet-paper dispensers in public bathrooms. They are also used covertly, in CCTV and police cameras. In these new contexts, successful recognition by AFR is increasingly intervening in a complex negotiation between recognition, identity and access. Alongside this expansion of AFR into everyday contexts, there is a growing realization that we are becoming reliant on machines looking at us - and, most importantly, perceiving and interpreting us - and making decisions that, ultimately, govern our existence.

Although AFR systems rely on a form of visual recognition, something about their processes is also paradoxically opposed to forms of looking, as is apparent in the example of the biometric turnstile at Helsinki airport given above. Often the operations of biometric scanning and identity verification occur within an invisible field and through processes that the human subject does not see. As a result, algorithmic processes of biometric recognition remain difficult to define, analyze and critique. A close analysis of the technology itself is necessary in order to understand how and by what means biometric identification occurs. Kelly Gates explains that the facial recognition technologies used in biometric identification "are being developed to address a fundamental concern of modern societies: the problem of 'disembodied identities' [...] the existence of visual and textual representations of individuals that circulate independent of the physical bodies." Yet this re-embodying of identities that occurs through a facial recognition operation occurs through a disembodied form of visual perception.

This book investigates AFR technology through an inquiry into its visuality, that is, into how an AFR process encultures a way of seeing and, as such, can be understood as a contemporary mode of perception by machine. It focuses on the 'recognition' part of automated facial recognition. Facial recognition through an AFR system contains three inherent tensions, which structure the following discussion. The first tension is that AFR technology is a form

1 Kelly A. Gates, Our Biometric Future: Facial Recognition Technology and the Culture of Surveillance (New York: New York University Press, 2011), 12. 
of machinic vision that is used to recognize us and yet, as I have mentioned, operates unseen and is, indeed, unrecognizable to those within its scope. In this way, AFR technology involves an invisible operation; its processes of recognition are inaccessible to us. Yet this operation increasingly governs people's lives within various institutional contexts. The second tension has to do with the question of the continuities and discontinuities between machinic visual perception and human visual perception. Many methods of AFR are designed and inspired by human processes of cognition and recognition, but to what extent do automated recognition processes replicate our own perceptual processes? To the extent that they do replicate these processes, in what ways does this replication relate, in turn, to a discourse of visuality? A third tension involves the relationship between the processes of recognition in an AFR system and the kinds of knowledge it may produce. A successful operation of automated recognition results in the production of (often actionable) information about the identity of a subject. An understanding of these processes of recognition may afford us a broader understanding of contemporary forms of identity production, and understanding these forms of identity production may allow us, in turn, to construct alternatives to the AFR process.

These tensions also suggest a critique of the notion of recognition inherent in AFR systems. In critically examining the notion of recognition, I intend to operationalize the term and use it as a tool to analyze AFR systems. These three tensions prompt a discussion of what recognition does and can possibly mean, and they problematize this notion. They lay out a general problematic of AFR technology as it relates to the context of its implementation and explored through the specificities of its technical processes. These tensions relate to broader issues of how machinic processes define visual perception as recognition. These tensions also provide an outline a line of inquiry into the relationship, in an AFR process, between seeing and knowing, that is, between how this process comes to produce not only the filtered data of information but also the knowledge that is accrued through the process of algorithmic learning in relation to the recognized subject. 
AFR technology is an example of the automatization of the labor of looking, which is taking place in a variety of contexts in the Information Age. The production of massive amounts of data from digital surveillance networks has made it that the ability to perform this labor of looking sometimes outstrips human capacities. AFR technology is utilized as a way of interpreting data and deriving meaning from it in order to produce "information," the primary product of this technology. Machine vision, or the automation of visual sense perception, has its origins in the controlled environment of the industrial factory, where it is used as a means of sorting and inspecting industrial parts and manufacturing flows. In the Information Age, machine vision technology has developed to be able to read measurable and quantifiable objects outside of the factory setting. In conjunction with the increasing use of surveillance systems, machine vision comes to focus on the processes and transactions relating to the flows of people in society. Similar to its use within the factory, biometrics, as one form of machine vision, functions in operations of inspection, that is, the sorting, selecting and surveilling of individuals in society. The biometric gaze turns toward the shifting, unstable and unruly forms of the body and reads these as if they were quantifiable industrial objects.

Facial recognition technology calls our attention to the site of the face not only as a means of identification but as a marker of identity. Inherent in the distinction between identity and identification is a politics of the face. In describing the site of the face as a particular mechanism of the political imaginary, Jenny Edkins writes, "the face in itself is a politics [...] that reflects and inscribes a particular intersection of two regimes of signs: the signifying and subjecting regimes." ${ }^{2}$ While the face can be measured, scanned and read like a sign, it is also expressive of the malleability and shape-shifting nature of the subjective experience of self. Faces are everywhere these days. In the so-called Age of the Selfie, one's self-portrait temporally and spatially situates oneself within the channels of various social networks. The selfie is an expression of the face as an embodiment of the self, depicting one's experience at a particular place and time.

2 Jenny Edkins, Face Politics (London: Routledge, 2015), 4. 
This may be why David Lyon calls on the face as an ethical starting point for a critical analysis of biometrics. ${ }^{3}$ He describes the site of the face as "resisting mere categorization" and claims its treatment is central to understanding how "one perceives the issues surrounding the appropriate conditions of self-disclosure." While the face is, as Lyon argues, expressive of an embodied social person and as such resists simple categorization, it is also a site of the body that is easily accessed, without consent, by biometric technologies. The prevalence of AFR technology is in part due to the fact that the face is a part of the body that tends to be visible in daily life and so is easily captured by surveillance mechanisms and CCTV. ${ }^{5}$ For example, in the arguments made in favor of AFR use in the ongoing "War on Terror," it has been stated that the only biometric data available on terrorists is their facial images. ${ }^{6}$

It has been noted by many programmers and developers of AFR technology that it confronts its own special challenges when it comes to both the detection and the recognition of human faces. The face is quite unlike the sorts of things typically the subjects of machine vision - subjects that are usually finite, measurable and geometrically fixed. As Lyon suggests, the face resists mere categorization not only through its expression of a subjective and embodied sense of identity but also physically, in its variability, multilayered forms of expression and constantly changing form, which resists the reductive methods of recognition used in AFR systems. In short, the face is a part of the body that escapes singular recognition. As such, the face as an object (and subject) of AFR technol-

3 David Lyon, "Surveillance as Social Sorting: Computer Codes and Mobile Bodies," in Surveillance as Social Sorting: Privacy, Risk and Digital Discrimination, ed. David Lyon (London: Routledge, 2003), 27-28.

4 Lyon, "Surveillance as Social Sorting," 27.

5 AFR is also in high demand because the use of facial images for identification purposes has a long history, and so there is a pre-existing infrastructure on which AFR can draw. Passport photos and criminal portraiture are examples of the use of the face and facial images as means of identification.

6 John D. Woodward Jr., Biometrics: Facing Up To Terrorism (Santa Monica, CA: Rand Corporation, 2001), 8, https://www.rand.org/pubs/issue_papers/ IP218.html. 
ogy provides a fascinating case study of machine vision, because it challenges the mechanisms of this technology both technically and theoretically.

A growing number of artists are working with facial recognition technologies in order to explore, confront and articulate sociopolitical issues raised by the use of these technologies. Artists engaging with facial recognition technology are uniquely positioned to articulate the visuality of this technology, and as such their works figure centrally in this study as objects of theoretical examination. Much of the development of AFR systems, and the surrounding discourse, has been guided by the aims of this technology in policing, military and market contexts. These artworks fill in a gap by providing a cultural translation of the technology, a translation that is often not discussed or explored by developers or programmers. Artistic engagements with facial recognition technology are able to articulate complex issues that AFR processes give rise to at the intersection of recognition, identity and representation. The strategies of artists engaging with facial recognition technology include appropriating the technology and decontextualizing its processes; in these ways, these artists allow for an engagement with this technology that not only problematizes its use but also imagines alternative outcomes of the technology and its processes.

There are three primary research questions that guide this study:

1) What is the process through which recognition is defined in the machinic form of vision used in an AFR method?

2) What historical continuities (and discontinuities) can we identify in these processes of recognition?

3) How do artistic interventions with facial recognition technology respond to, articulate and confront the implications of the use of this technology?

The general purpose of these questions is to achieve a broader understanding of AFR technology within the sociopolitical and cultural contexts in which it is used. These questions focus on the general problematic and central principle of AFR technology by asking how 
recognition can be defined through a technical process. Although these questions approach a general problematic of recognition in AFR technology, they also get at the specificities of how this technology operates. These questions reflect on the technology itself, on how a technical process encultures a way of seeing through recognition. These questions also relate the ways in which the face is represented through an AFR method to a discourse of visuality, namely, historical practices of facial representation and portraiture. This line of inquiry acknowledges that the forms of visuality involved in an automated recognition process are a central source of the knowledge this technology produces. The ways in which the face is represented in this process of recognition are how an AFR method itself comes to know a face. In addition, through a process of successful recognition, an AFR method produces knowledge in the form of the identity and identification of the person being recognized. In referring to examples of artistic interventions by contemporary artists, this analysis presents an inquiry into the visuality of automated facial recognition and the ways in which meaning is both produced and made malleable by AFR technology.

\section{Cultural Analyses of Biometrics: Previous Scholarship}

Biometrics and, in particular, AFR technology have increasingly come to be applied within the contexts of risk mitigation and security practices for the identification and recognition of individuals. Biometrics utilizes advanced visual technologies, such as digital sensors, to scan, measure and capture parts of the body, their forms and surface patterns. The uniqueness of body parts, such as finger prints, an iris or a person's face, allow these technologies to ascertain an individual's identity. The practice of using the body as a sign of identity has a long history that dates back to the mid-1800s, beginning with the practice of using a hand imprint to seal a contract, a practice that has a more recent analogue version in the collection of the fingerprints of grade-school students. In the 1980s, the term "biometrics" began to be used to describe the automated systems of human recognition then being developed; in the 1970s, the field 
had been known as "automated personal identification." What has changed with more recent developments in biometrics is that these practices are now being digitized, which includes the use of algorithms to read collected bodily data and the use of biometric technologies in conjunction with growing and widespread networks of surveillance. Alongside the increasing use and continued development of contemporary biometrics, there has been a corresponding increase in critical academic scholarship in the humanities that has studied biometric and AFR technology through a cultural lens. In the last ten years, scholars from the fields of sociology, surveillance studies and media and communications have produced work that articulates the social, political and cultural implications of society's increasing dependence on biometrics. The scholars included in this brief overview of previous literature for the most part critically approach the use of biometric and AFR technology through analyses of their technological development and the systemic contexts and implications of their implementation. They have analyzed the technology through theoretical frameworks that draw on a range of discourses, including sociology, surveillance studies, science and technology studies, post-colonial theory and gender and feminist theory.

One of the first scholars to adopt a sociological approach to biometric technologies was David Lyon, who in 2001 described biometrics as one aspect of the growing ubiquity of widespread surveillance practices that function as a method of "social sorting," that is, as a way of "categorizing populations and persons for risk assessment [...] [in] attempts to minimize risk, by discovering - preferably in advance - who is likely to break the law, buy the product, or seek the service." Lyon's use of the term "social sorting" highlights the "classifying drive" as a central function of surveillance and biomet-

7 James L. Wayman, "The Scientific Development of Biometrics over the Last 40 Years," in The History of Information Security: A Comprehensive Handbook, ed. Karl de Leeuw and Jan Bergstra (Amsterdam: Elsevier, 2007), 263-74.

8 David Lyon, "Facing the Future: Seeking Ethics for Everyday Surveillance," Ethics and Information Technology 3, no. 3 (2001): 172, https://doi. org/10.1023/A:1012227629496. 
ric technologies. ${ }^{9}$ Rather than raising issues of privacy concerning the individual, Lyon brings the discussion of biometric and surveillance practices into the social realm, examining their use as mechanisms of neoliberal policy that enforce social division and categorization and imply unequal access and distribution. Lyon's analyses of biometrics establish a framework through which to approach the social power of its information production.

Lyon's critique of biometrics as a surveillance practice highlights its unequal implementation and effects on vulnerable and marginalized parts of the population. This topic is expanded on in the work of Simone Browne and Shoshana Magnet, both of whom analyze the dialectics of recognition in biometric technology and the ways it is directed by normative categories of identity. Both scholars the ways in which applications of biometric technology deny a subjectivity, and they associate the limitations of the technology with a limit to notions of identity. Biometric technologies thus enact a negation of certain individuals based on their race, gender and/or economic status while verifying the identities of certain others. Through the use of different theoretical frameworks and discourses, both Browne and Magnet recognize that there is a cultural logic embedded in biometric technology, and their works actively critique this logic.

In her book Dark Matters: On the Surveillance of Blackness, Browne draws on the discourse of post-colonial theory, referencing the work of Franz Fanon and his concept of "epidermalization" to approach the power dynamics of a biometric, disembodied gaze. She defines the concept of "digital epidermalization" as "the exercise of power cast by the disembodied gaze of certain surveillance technologies [...] that can be employed to do the work of alienating the subject by producing a truth about the racial body and one's identity (or identities) despite the subject's claims." ${ }^{10}$ Browne's analysis outlines historical continuities that can be found within biometric and surveillance practices by placing these in direct dialogue with an archive

9 Lyon, "Surveillance as Social Sorting," 13.

10 Simone Browne, Dark Matters: On the Surveillance of Blackness (Durham, NC: Duke University Press, 2015) 110. 
of transatlantic slavery and the history of the control, regulation and surveillance of black bodies. Through her analysis, Browne describes the inherent violence that occurs through the enactment of institutional recognition by the state.

Magnet's book When Biometrics Fail: Gender, Race and the Technology of Identity adopts a feminist theoretical framework. ${ }^{11}$ The point of departure for her study is a call for a broader and more precise vocabulary for defining a notion of failure in relation to biometric technology. In this way, Magnet aims to counter an acceptance of failure as technically productive: technical failures are usually accepted as means to an end, leading to further developments and eventual success. Instead, Magnet defines a sociological framework of failure according to which, when biometrics technologies "over target" and "fail to identify," these failures are defined as sociologically counterproductive, as excluding certain communities and resulting in the inaccessibility of resources for certain segments of the population. Magnet argues that the failure of the science of biometrics lies in the fact that it is a technological implementation of gendered and racialized norms, essentially codifying "existing forms of discrimination"12 and thereby failing to recognize the complexity of bodily identity.

Magnet's analysis may be seen alongside the work of other scholars who focus on the technical limitations of biometric technologies. Kelly Gates's book Our Biometric Future: Facial Recognition Technology and the Culture of Surveillance, in particular, challenges the legitimacy of AFR technologies by examining the gap between the claims made about their viability and their actual capabilities. ${ }^{13}$ Drawing on communications theory, Gates gives an in-depth account of the political and economic constellation of influences governing the development and implementation of AFR technology. Gates argues that the claims of technical precision made on behalf of AFR technology by the biometrics industry and government

11 Shoshana Magnet, When Biometrics Fail: Gender, Race and the Technology of Identity (Durham, NC: Duke University Press, 2011).

12 Ibid., 9.

13 Gates, Our Biometric Future, 98. 
officials make its implementation appear inevitable. Furthermore, these claims mean that AFR technology is often given precedence over other forms of intelligence gathering. In examining the actualities and limitations of the technology, Gates's study aims to disrupt this trajectory and to provide a more down-to-earth account of its advanced capabilities.

Gates explores how AFR technology was framed as the solution to the particular challenges of the post-9/11 period. She describes the narrative advanced by the research agency of the US Department of Defense (DARPA): that the war on terror involved a new kind of enemy, an "unidentifiable" enemy who thus implied a new form of national vulnerability. ${ }^{14}$ Gates explains that, in virtue of AFR technology's ability to produce a kind of identifiability for these enemies, and thereby provide a sense of certainty in the post-9/11 geopolitical landscape, the need to deploy this "expensive, new, high-tech surveillance technology [...] seemed self-evident. ${ }^{115}$ Gates's study shows how the development of AFR is based on the fact that this technology has been framed as a solution to the political and military challenges faced within the contemporary geopolitical landscape.

Numerous scholars have approached an analysis of biometrics through the lens of geopolitics. One example is Btihaj Ajana, a scholar working in the area of digital cultures who approaches an study of biometrics through the theoretical discourse of biopolitics, drawing on the work of Michel Foucault, Giorgio Agamben and Nikolas Rose. ${ }^{16}$ Ajana argues that biometric technologies enact a form of biopolitics and result in the construction of politicized identities along geopolitical lines. For example, Ajana examines the binary of the "asylum seeker" and the "neoliberal citizen," which in turn comprises a whole set of practices that govern the individual: hierarchical power relations marginalize asylum seekers, trampling their basic rights, especially their rights to move freely, and also empower the neoliberal citizen, at the opposite end of the geopolitical spec-

14 Ibid.

15 Ibid.

16 Btihaj Ajana, Governing through Biometrics: The Biopolitics of Identity (Basingstoke: Palgrave Macmillan, 2013), 34-44. 
trum, with a "surplus of rights." ${ }^{17}$ Louise Amoore, a scholar in the field of political geography, also approaches an analysis of biometric technology within the context of geopolitics. She does so by drawing on a conceptual framework from the field of geography. In her concept of the "biometric border," she casts biometrics as a way of constructing a new kind of geopolitical border, which is made manifest through the mapping and measuring of the body. ${ }^{18}$ Biometrics does not analyze abstractions but maps the actual biophysical patterns of the body and, by doing so, inscribes the body with institutional divisions and demarcations. As Amoore writes, "In effect, the biometric border is the portable border par excellence, carried by mobile bodies at the very same time as it is deployed to divide bodies at international boundaries, airports, railway stations, on subways or city streets, in the office or the neighbourhood."19

The work of these scholars presents a range of diverse approaches to the cultural, social and political implications of biometrics and AFR technology. These scholars articulate some of the most pertinent and salient conclusions of the cultural and social critique of these technologies. They explore how the development and implementation of these technologies depends on a certain way of framing both the solution and the problem. Overall, these scholars' works undermine the claims of neutrality and precision so often made on behalf of biometric and AFR technologies. These scholars recognize that biometrics and AFR technology directly participate in decision making, with wide-ranging social and political implications. They look into the social and economic interests behind the development of the technologies as well as the results in terms of contemporary constructions of institutional identities. These cultural analyses of biometric technologies recognize that the implementation of these technologies brings about not only a technological but a cultural shift. By bringing to bear a diverse range of theoretical discourses

17 Ibid., 2.

18 Louise Amoore, "Biometric Borders: Governing Mobilities in the War on Terror," Political Geography 25, no.3 (March 2006): 336-51, https://doi. org/10.1016/j.polgeo.2006.02.001.

19 Ibid., 338. 
in their discussions of biometric technology, these scholars articulate some of the cultural implications of the implementation of this technology. A central critique of biometric technologies advanced by these scholars is that these technologies draw their notions of identity from the agendas of the institutions that implement them, and force subjects to accord with these notions. My own analysis takes this central critique as its point of departure. But, in contrast to the works discussed above, which examine the dominant social and political narratives that surround the development of biometric technology and its present application, I look at the empirical material of the technology and the technical processes themselves as a window into a cultural logic of visuality. This, in turn, may provide us with a source of critique and further insight into the limitations of this technology with regard to notions of identity and knowledge formation.

\section{Language and Visual Artifacts: Empirical Material}

As I have explained, previous cultural analyses of biometrics and AFR technology have focused on the contexts of their development and implementation. And while this is necessary in order to problematize the use of these technologies, these studies tend to overlook the specificities of AFR technology. There has been little scholarship from within cultural studies and the humanities more broadly on the ways in which AFR actually performs recognition and how this may constitute and enculture a mode of perception. More often, the specificities of the technical processes of recognition are discussed and debated within the field of the computer and cognitive sciences, separated from any cultural investigation. When AFR technology has been discussed within cultural studies, it is for the most part treated as a monolithic method rather than as a variety of different methods that have evolved in specific ways. When the topic is scrutinized in this way, individual AFR methods are largely ignored. In contrast to this approach, I choose to focus my own analysis on a specific AFR method. 
My interest in examining the visuality of AFR has led me to focus on method that uses an eigenface approach to recognition, an approach that was developed in the early 1990s and is considered the first successful method in AFR technology. Many AFR systems are difficult to scrutinize because the technology is usually considered proprietary software. ${ }^{20}$ The eigenface approach, now considered a dated method, was open source from its inception. Because of its simplicity, it is often still used as a training tool by computer science students, so examples of its use are widely available on the internet. There are two primary reasons for my interest in this method. Firstly, the eigenface approach is considered a holistic method: that is, it takes into account the entire face, rather than isolated features of the face, and is, as such, designed to emulate human facilities of facial recognition. Secondly, as a part of its algorithmic processes of recognition, the eigenface approach was designed to produce an image, a visual artifact through which it is possible to enter into a visual analysis of its processes of recognition. The success of the eigenface approach to recognition made it a benchmark for the AFR methods that developed subsequently. And although it is now considered a somewhat dated method, its creation still remains an inflection point in the history of the development of AFR, shifting, for a moment, the direction of the technology toward holistic and pictorial processes of facial recognition. The eigenface approach is a simple method. In its simplicity, it reveals the technical processes that structure a successful AFR recognition operation. This basic structure can still be found in the more sophisticated AFR methods that have since been developed.

My investigation into biometric facial recognition technology differs from the theoretical frameworks outlined in the previous section in that it analyzes AFR technology in relation to discourses of visuality and machinic vision. Nevertheless, this investigation does draw on the conclusions and some of the central conceptualizations of previous cultural analyses. These conceptualizations act

20 Lucas Introna and David Wood, "Picturing Algorithmic Surveillance: The Politics of Facial Recognition Systems," Surveillance and Society 2, no. 2/3 (2004): 185, https://doi.org/10.24908/ss.v2i2/3.3373. 
as useful prompts, providing inroads into the analysis of the visuality of an AFR recognition process. For example, drawing on Lyon's aforementioned critique of biometrics and surveillance practices as enacting a form of "social sorting," I ask what technical means AFR methods employ in this sorting process. How does this sorting equate to a form of vision? How are contemporary modes of perception reduced to a sorting mechanism? As this line of questioning makes clear, this analysis approaches AFR technology by specifically problematizing a visuality found in its processes, a visuality that has social and political implications.

As I have mentioned, the eigenface approach is an earlier, "pictorial" algorithmic method that reads the face holistically (rather than a feature extraction method that measures the distances between features of the face). What sets the eigenface approach apart from other methods is that, as I mentioned above, it produces an image as a part of its algorithmic processes. The eigenface image and, importantly, the algorithmic recognition processes that produce it, constitute the central departure point of this study. The production of this image was originally a way of providing the programmer with a way to fix bugs in the algorithm. This image appears as a phantom-like blur of multiple overlapped faces, which, to human eyes, lingers on the threshold of recognition. While the eigenface image remains blurry to human vision, it contains a wealth of information that, for the processes of perception by the machine, equates to a form of clarity. The image provides a visual artifact that allows me to bring AFR processes into discussion with visual and cultural theory and discourses on machinic vision. I do this in two ways. Firstly, as I will explain in more depth in the theoretical section of the introduction, I present this image as an example of the changes in the role of the image as information brought about by the advent of digital networks. Secondly, I positioned the eigenface image within a discourse of facial representation in the art historical genre of portraiture. Taken out of its original context of production, that is, as a tool for programmers, I understand the eigenface image to be a machinic production of a portrait, depicting the human face from the perspective of machine. I investigate this in depth in the first part of this book, which relates the eigenface 
image to a historical antecedent: the composite portrait. This line of analysis continues in the second part of the book, which looks at artistic interventions and discusses portraiture in the age of AFR technology.

Another, related source of empirical material is provided by the scientific texts that describe the eigenface method. I was first introduced to the eigenface method by reviewing scientific journal articles that outline the development of and experimentation with AFR methods by programmers and students in the field of computer sciences. The central texts for the analysis of the eigenface method are two articles written by its developers, Matthew Turk and Alex Pentland. Aside from the articles written by Turk and Pentland, which record the initial findings of experiments using the eigenface method, Turk later wrote another article, "Twenty Years of Eigenface," which provides further insight into the impetus behind its original development and its relationship with other methods of AFR technology. ${ }^{21}$ I have also drawn on the work of the computer scientists Lawrence Sirovich and Michael Kirby, whose work preceded that of Turk and Pentland. Sirovich and Kirby applied the primary representational mechanism used in eigenface, Principal Component Analysis, to facial images, which influenced Turk and Pentland's work. ${ }^{22}$ These works are the primary scientific texts concerning the eigenface method. The method has become a popular algorithmic training tool for students of computer science, and there are numerous published scientific journal articles and blogs by computer science students and programmers that build on the method. These provide an endless source of information about eigenface images and a wealth of examples of such images. I have made use of some of these texts and images; because many of the blogs were written in such a way as to be accessible to laymen such as myself, these proved to be particularly useful.

21 Matthew Turk, "Over Twenty Years of Eigenface," ACM Transactions on Multimedia Computing Communications and Applications 9, no. 1 (October 2013): 1-5, http://dx.doi.org/10.1145/2490824.

22 Lawrence Sirovich and Michael Kirby, "Low-dimensional Procedure for the Characterization of Human Faces," Journal of the Optical Society of America 4, no. 3 (April 1987): 519-24. 
A central challenge I confronted in seeking to understand the eigenface method through relevant scientific texts was that I had to understand specialist language from outside of my own field of expertise. Not only is there an abstraction of the image that occurs through the eigenface process; in analyzing the texts that outline this method, I also confronted an abstraction in the language used to describe it. These scientific journal articles communicate the method in four different forms: text, mathematical equations, photographic and video images and graphs. In the course of my analysis, I found the images to be most relevant. The images corresponded to specific equations that explained more fully, for a specialized computer-science audience, the underlying procedures. Mathematical equations relating to the method and its processes figure prominently in the texts: for example, 27 times in the primary text by Turk and Pentland on the eigenface method. ${ }^{23}$ It is important to note here that I do not include any mathematical equations in my own research; I lack knowledge of programming, and I am writing a cultural analysis within a field of the humanities for an audience within this discipline. Instead, I have focused on the language and the words chosen to describe the process and, of course, the eigenface images themselves. The captions written by the authors of the scientific articles, which at times take into account the aesthetic aspect of the images, were also of importance for this study, for they express what was considered important in the image and supplement an explanation of the algorithmic process. Inevitably, the language as well as the images used by the developers became a central part of the empirical material.

I have tried to bring to bear a cultural theoretical framework and concepts on a technical phenomenon that has predominantly been described in a specialist language and through mathematical equations. I have thus faced a challenge in attempting to communicate the central mechanisms behind this process, as I understand them, without parroting the specialist language of the original sources and while also being careful not to oversimplify this explanation.

23 Matthew Turk and Alex Pentland, "Eigenfaces for Recognition," Journal of Cognitive Neuroscience 3, no. I (Winter 1991): 71-86. 
The language used in the humanities contrasts with that in the so-called "hard sciences," such as the fields of applied mathematics and computational sciences, specifically when it comes to the site of knowledge production. Scientific literature often describes phenomena from an assumed objective and distanced standpoint of observation, while, in contrast, as a humanities scholar, my own approach is explicitly interpretative, and it often requires an explanation of my own and others' specific historical, cultural and socially situated vantage points. In short, in my observations, the site of knowledge production includes an "I," and I freely admit my own limitations in confronting the specialist language used in these scientific texts, which make up a large part of the empirical material for this study. ${ }^{24}$

I refer to in what follows include "The Scientific Development of Biometrics over the Last 40 Years," by James L. Wayman, from The History of Information: A Comprehensive Handbook, and the recently declassified reports by computer scientist Woodrow Wilson Bledsoe, which concern some of the first attempts to develop AFR technology, funded by the CIA. ${ }^{25}$ These supplemental texts have helped me to explore further the broader contexts in which AFR technology has been used and developed. They also provide further information that situates the success of the eigenface method within the developmental trajectory of AFR technology.

Another primary source of empirical material in this study is the collection of artists' works explored in the second part of the book. The examples of artistic interventions included in this part bring out particular issues to do with the implementation of facial recognition technology in society. Each artist relates the technology of facial recognition to specific discourses. Thomas Ruff's study of the identification portrait as an artistic object creates a bridge between

24 Thank you to Anthony Paré and his "Anatomy of a Genre" course for insight into this difference between academic perspectives with regard to situatedness. For more info, see Anthony Paré, "Rhetorical Genre Theory and Academic Literacy," Journal of Academic Language \& Learning 8, no. I (2014): A83-A94.

25 See footnote 7 in chapter 2 for more details about how these reports became declassified. 
AFR technology and a discourse on photographic portraiture. AFR technology still relies on photographs or video-still captures of the face and as such on a form of representation that connects back to the indexical relationship between identity and portraiture and the use of the photograph as a scientific document. Ruff's work with the construction of faces in his series andere Portraits situates the composite portrait into the realm of art. As such, his work serves to connect the analysis of the eigenface method with the themes of the second part of the book. Zach Blas's work brings AFR technology into a discussion with discourses of identity politics. His work confronts the gaze of AFR technology, and his sculptures materialize a contemporary strategy of activism through collectivism. Blas's artwork highlights the social and political contexts in which AFR technology is implemented and the enmeshment of automated recognition practices in the construction of contemporary identities. Trevor Paglen's work is a study of visuality in light of discourses concerning the growing ubiquity and invisibility of algorithms in society. Paglen's work not only disrupts how contemporary meaning is produced through algorithmic recognition processes but also explores the role of imagination. This collection of artworks explores three essential aspects of the influence and role of contemporary AFR technology: representation, identity and knowledge production. With the inclusion of these artworks, I hope to show that art has an important role to play as a source of theoretical reflection on contemporary technologies, as well as offering a means of exposing their dangers and exploring the possible alternative futures resulting from these technologies.

\section{Temporalities - History as Critical Inquiry: Methodology}

A primary method of historicization guides the critical analysis of the eigenface algorithm in the first part of this study. Eigenface is related to a historical antecedent, composite portraiture, which was invented by the anthropologist, statistician and founder of eugenics Francis Galton in the 1880s. These two methods are connected by the idea of merging vision with statistics in order to provide a 
mechanism of recognition. The method of historicization as a tool for critically analyzing AFR technology relies on a concept proposed by African studies and surveillance scholar Simone Browne. Browne calls for a "critical biometric consciousness," which includes ways of developing critical strategies that can reveal and allow us to scrutinize the technical development and socio-cultural implications of biometric technologies. ${ }^{26}$ Central to this call is an approach to biometric technologies that contrasts with its treatment as objective and without history. This approach is directly opposed to the widely held belief that algorithms and biometric scanning exist as privileged, ahistorical practices of information technology that are objective and precise, a belief that validates the continued development and use of these technologies. Browne describes one method of developing a "critical biometric consciousness": tracing historical practices and antecedents that inform the social dynamics and technical development of contemporary biometric technologies. ${ }^{27}$ In tracing the socio-historical lineage of the facial representation processes found in the eigenface method, this analysis answers Browne's call. By linking the eigenface image with the historical antecedent of composite portraiture, we are able to reveal the cultural dynamics of facial representation that inform automated processes of recognition. In historicizing the eigenface image in this way, this study reveals not only a logic of recognition based on the merging of statistics with vision but also an embedded and situated cultural logic of facial representation within the eigenface's algorithmic processes.

The method of historicization in this analysis is inspired by recent scholarly work that has critically engaged with overarching, general terms by tracing the histories behind their meanings. Two key texts that have influenced this study are the book Objectivity, by Lorraine Daston and Peter Galison, and John Durham Peters's work on the term information, specifically in his article "Information:

26 Browne, Dark Matters, 116.

27 Ibid., 116-18. 
Notes Toward a Critical History." ${ }^{28}$ Both of these works pursue a method of historicization, tracing the cultural meaning behind the practice-based terms "objectivity" and "information" as a means of demystifying their contemporary position as transparent and "omnipresent idols"29 and grounding their meaning in actual use and in modes of discourse. Like the scientific illustrations that appeared at the turn of the last century, recent technological developments and processes of information production are often, in the fields of cultural or aesthetic inquiry, treated as possessing a kind of objectivity. Often it is only the distance of history that allows us to engage in a more critical way with a scientific production that had been accepted as neutral in its own time. In tracing the historical continuities of the culturally embedded recognition practices found in an AFR method, I argue that we may contest the assumed scientific objectivity of the technological present.

By relating the eigenface algorithm to a historical antecedent, the composite portrait, I put two sets of images into dialogue with one another: one the result of the representational mechanisms of the eigenface algorithm, and the other Galton's historical, photographic composite portraits. The relationship between these two sets of images involves a history of what I consider a way of seeing - a mode of perception that is based on statistical logic. In relating these two images, I am not trying to trace the origins of eigenface but rather relating it to a particular historical instance in order to reveal how its mode of perception is embedded within a specific cultural ethos. One thought that is central to my own approach to relating these two images comes from Walter Benjamin's “Theses on the Philosophy of History" (the last text he wrote before his premature death). Benjamin writes: "The past can be seized only as an image which flashes up at the instant when it can be recognized and is never seen again [...] For every image of the past that is not recognized by the present as one of its own concerns threatens to

28 John Durham Peters, "Information: Notes Toward a Critical History," Journal of Communication Inquiry 12, no. 2 (1988): 9-29, https://doi.org/ 10.1177/019685998801200202.

29 Ibid., 19. 
disappear irretrievably." ${ }^{30}$ In my analysis, the composite portrait appears as a pictorial reference to a cultural ethos of social classification from the past that, through contemporary practices of biometrics, finds a foothold in the present. The composite portrait is a contextually loaded image; embedded within it is an impulse to construct a social taxonomy, as part of Galton's broader project of eugenics. This particular history is recalled by the classifying impulse of contemporary AFR technologies and is visualized through the eigenface image. In relating these two images, I do not mean to suggest that the historical relationship between them is self-evident or that it exists on its own. Rather, to quote Benjamin again, "To articulate the past historically does not mean to recognize it 'the way it really was' (Ranke). It means to seize hold of a memory as it flashes up at a moment of danger." ${ }^{31}$ It is in this spirit that I hold up the eigenface image and the composite portrait as objects of study connected by a relationship in which the past informs the present: the algorithmic processes used in the eigenface method are embedded with historical practices of racially charged classification, which makes clear the present danger posed by AFR technology and suggests a critique of its limitations and entanglements with regard to notions of recognition and identity. Tracing this historical link between the practice of composite portraiture and the representational mechanism within the eigenface algorithm also reveals a link between discourses in photography - in its role in science and art - and the forms of representation that are found in current algorithmic processes.

The second part of this study draws on contemporary artists whose work bears on practices of facial recognition. The artists Thomas Ruff, Zach Blas and Trevor Paglen have each produced works that function within this study as further sources of theoretical analysis, experimenting with and problematizing processes of facial recognition. These artists were chosen because of their

30 Walter Benjamin, "Theses on the Philosophy of History," in Walter Benjamin, Illuminations, trans. Harry Zohn (New York: Schocken Books, 2007), 255.

31 Ibid. 
explicit concern with some of the political and social implications of facial representation and recognition technologies. Their work supports an overarching narrative that runs through this analysis, a narrative that is concerned with the visuality and representational mechanisms of facial recognition. The artworks articulate the cultural contexts in which facial recognition technology intervenes, and they pose, in particular, the central criticism of these technologies: that they reinforce normative categories of identity through reductive, technical procedures. I have chosen artworks composed using a variety of mediums in order to explore a range of responses to the implications of facial recognition. Ruff, Blas and Paglen work in the mediums of photography, sculpture and algorithmic-generated prints, respectively. The artists' works included within this study serve to supplement a central conceptual motif of the analysis of eigenface (one artist, Paglen, directly references the method in his work) through their engagement with and reformulation of composite forms of the face. Two of the artists, Blas and Paglen, have also produced written scholarly work alongside their artistic practices, and I have also made reference to this work. The concepts that these artists are working with are often articulated explicitly in these texts. I also had informal discussions with Blas and Paglen in person, which gave me further insight into the contexts and cultural circles in which they engage with their work. I conducted email interviews with Ruff, and I also refer to existing interviews with him that are included in catalogues of his work. All of the impressions I gained, both through written texts and in-person dialogue, provided further material for and insight into the approach they take towards facial recognition in their work. A primary motivation in choosing the artworks included in this study is that they use art as a vehicle for imagining alternatives to the technologies that increasingly govern our social life.

The two parts of this study experiment with the separate temporal trajectories of past and future. In historicizing the eigenface method by relating it to the composite portrait, this analysis consciously counters a preemptive logic of the technology and, instead, points "backwards" toward the past as a source for informing the future. The first part of this study, which analyzes the eigenface 
method, may be understood as referring primarily to the past, while the second part points toward the future through an exploration of contemporary artistic interventions and a discussion of possible alternative responses.

\section{Machinic Observer - Seeing as Recognition: Theoretical Framework}

Because this study focuses on the visuality of AFR technology, I relate the empirical material under discussion here to the work of contemporary scholars of the image who have sought to understand how visual sense perception may be understood as a machinic process. Most of this work relates to a theme in visual culture theory concerning the "disembodiment of the eye" that occurs through the use of visual technologies and is often analyzed through referencing its expression in works of art. I argue that this theorization is useful in analyzing contemporary advanced visual technologies, but also that it has its limitations. Some of the ideas derived from this theory do not figure directly in the analysis, but I mention them here because they have inspired my thinking and my central approach to the analysis generally.

I'd like to begin an approach towards understanding machine vision with a reference to a scene from a film. In Charlie Chaplin's Modern Times, the protagonist, played by Chaplin, is working in a factory and becomes enmeshed in the rhythm and movements of the industrial line. ${ }^{32}$ His arm movements become one with the rhythm of the conveyer belt that brings him more and more objects to clonk. Later on, his entire body gets entangled in the workings of an enormous machine that appears like the insides of a clock, complete with springs, cogs and levers. Chaplin's body, along with all its senses and movement, has been swallowed into the belly of the industrial machine. The factory of Modern Times depicts an era of pro-

32 "Chaplin Modern Times - Factory Scene (late afternoon)," YouTube video, 4:13, posted by "Olaf V/s Minions," September 5, 2015, https://www.you tube.com/watch?v=HPSK4zZtzLI. 
duction in which the human body as a whole was employed within the industrial labor force of mass manufacturing, albeit in order to carry out specialized tasks. In contrast, thinking about how a person might become enmeshed in the labor and production processes of today's Information Age, it is clear that the dominant part of our body that is forced to work is not the arm but rather the eyes. Instead of the entire body becoming enmeshed in the rhythm of the industrial machine of production, the eye and the sense perception of sight become entangled within the operations of the information machine. The labor of looking has become a central demand within multiple contexts in the Information Age, where the challenge is often in making things visible, and thereby known, within a sea of data. Widespread surveillance practices have made it that the amount of data produced now far outstrips human capacities of understanding. Algorithms have been developed to codify this visual labor, to inspect and sort through the mass of data, to make "sense" through the automation of a form of sense perception. In this scenario, vision as a form of sense perception connected to the production of knowledge and meaning has become aligned with the logic, operations and rhythm (or temporalities) of the machine.

It is important to begin with a concept of machine vision, as it is a central concept that underlies my whole approach to analyzing AFR technology, as being a contemporary example. I am interested in AFR technology as an object of study because of the way it codifies and automates visual sense perception within an operation of recognition. Scholars have theorized machine vision within a discourse of art history and visual culture. John Johnston has outlined a concept of "machinic vision," which he defines as "not only an environment of interacting machine and human-machine systems but a field of decoded perceptions that, whether or not produced by or issuing from these machines, assume their full intelligibility only in relation to them. ${ }^{.33}$ In his use of the term "machinic," Johnston is drawing on the work of Gilles Deleuze and Felix Guattari and their understanding of the term as suggesting an "assemblage," that is,

33 John Johnston, "Machinic Vision," Critical Inquiry 26, no. I (Autumn 1999): 27, https://doi.org/10.1086/448951. 
"a type of working relationship among heterogeneous elements." ${ }^{34}$ Deleuze distinguishes this understanding of the machine, as a binary opposite of the human, or the organic. Johnston describes "machinic vision" as a disembodied perspective that derives from an assemblage of machine and human practices. He references expressions of a machinic vision, for example, through art in the paintings of Francis Bacon and in cinema through the work of Dziga Vertov. Again drawing on Deleuze, Johnston describes a machinic vision as rejecting a centered world view and a "phenomenological point of departure." ${ }^{35}$ Instead, Johnston says that machinic vision is a "pure vision of a non-human eye, of an eye which would be in things." In this way, the act of seeing is liberated from an anchored point and becomes mobile - it may be found in objects, take on a molecular form and operate beyond human scale.

Two key movements of Deleuze's definition of the "machinic" that Johnston applies to vision are deterritorialization and reterritorialization. Johnston describes that the former occurs when a form of visual perception is freed from the person that is doing the seeing, and the latter is when that seeing is "recoded," that is, recontextualized and expressed in new form and, as a result, produces new meaning. Johnston argues that what must be understood is this recoding. He outlines the problem of approaching these moments and expressions as they occur in forms other than the traditional forms of art he previously mentioned, particularly when they occur in digital images. He states that:

in order for there to be a deterritorialization and thus a decoding of perception, there must be a movement toward the outside of an assemblage and beyond its coding apparatus, a movement carrying us into a zone where images become indiscernible, often as a result of particle-ization of elements [...] But for the digital image there is no outside, only the vast telecommunications networks that support it and in which it is instantiated as data. Instead of an outside,

34 Ibid., 28.

35 Ibid., 34. 
the digital image seems only to have an electronic underside [...] which cannot be rendered visible. ${ }^{36}$

Johnston outlines the challenges of a circulatory and self-referential system found with images within digital networks. In the following analysis, I address the problem of revealing an "underside" of the digital image by basing my inquiry on a visual artefact of an AFR process. Specifically, I will approach the challenges posed by digital networks through a discussion on the recontextualization of the algorithmically produced eigenface image and through the examination of the work of artists who have relied on non-digital mediums. The movements that Johnston define are key in this analysis to understanding how an AFR process deterritorialize visual perception in an algorithmic process and reterritorialize it in an operation of recognition. I specifically problematize the "recoding" of vision through the eigenface algorithm and its ability to produce new meaning in terms of the identity of the subject being recognized.

Paul Virilio is someone who figures centrally in the theorization of machine vision and although his conceptualization on the topic does not appear in this analysis, he has nonetheless inspired my approach and thinking and therefore needs to be mentioned here. His book The Vision Machine predicted the widespread implementation of machine vision in society. He writes, "Unless you are Lewis Carroll, it is hard to imagine the viewpoint of a doorknob or a button on a cardigan. Unless you are Paul Klee, it is not easy to imagine artificial contemplation, the wide-awake dream of a population of objects all busy staring at you. ${ }^{37}$ In his brief and singular reference to an "artificial contemplation," Virilio refers to works of literature and art production as mediums through which one can imagine and communicate the life of objects and in particular the perspective from which objects can perceive a subject. My own methodology is inspired by Virilio's suggestion that mediums outside of techni-

36 Ibid., 39.

37 Paul Virilio, The Vision Machine, trans. Julie Rose (Bloomington, IN: Indiana University Press, 1994), 137. 
cal research might allow one to make sense of "artificial contemplation." Given the ways in which an automated process of vision considers and makes meaning of physical phenomena, this seems a promising suggestion. This analysis considers Virilio's suggestion in relation to algorithmic processes, and it attempts to formulate a narrative around algorithmic contemplation as it relates to AFR technology. As such, I like to bring Browne's aforementioned argument for a "critical biometric consciousness" into dialogue with Virilio: a kind of discourse between a social consciousness confronted with this algorithmic contemplation, whereby, at this intersection, it may map a critical terrain on the ways in which algorithms can give accounts to themselves through the perspective of an automated sense perception.

The artist Harun Farocki is another figure whose conceptualization figures in this analysis in particular his work with machine vision. Farocki's concept of "operational images" has been influential in understanding the ecology of images that result from the output of machine vision operations. He defines the term as "images that do not represent an object, but rather are part of an operation. ${ }^{338} \mathrm{Fa}$ rocki has described the operational image as an image that is unlike traditional images in that it is made neither to entertain nor to sell; operational images, he says, "are information and not really images. ${ }^{139} \mathrm{He}$ further describes the operational image as implementing a new visual regime where images are made by machines for other machines and, "the aesthetics of which are not intended."40 The operational image poses a shift in the role of images from representational to operational, and as such ascribes to images an agency within a prescribed function. This is particularly pertinent to both parts of my analysis, but especially to the analysis of eigenface, which begins with the algorithmically produced eigenface image. The eigenface image is an example of an operational image, for it is

38 Harun Farocki, "Phantom Images," trans. Brian Poole, Public 29 (Spring 2004): 17 [12-22].

39 Harun Farocki, War at a Distance, video (Berlin: Harun Farocki Filmproduktion, ZDF, 2003).

40 Ibid. 
generated only through its recognitive operation. Yet, the eigenface image overlaps the two registers of representation and operation in that it harbors both. The eigenface image is part of the operation of recognition in machine vision yet it is also an image that depicts the representation of faces (or the representational mechanism) within an algorithmic process.

Another aspect in framing the theoretical approach of this analysis, is relating an understanding of machine vision within a discourse on a historical construction of vision. Jonathan Crary's book Techniques of the Observer asks pertinent questions and provides certain points of departure that have contributed to the development of this outlook. Crary attends to the phenomenon of the observer (rather than to the artistic image as a source) in investigating vision as a historical construct. He states:

For the problem of the observer is the field on which vision in history can be said to materialise, to become itself visible. Vision and its effects are always inseparable from the possibilities of an observing subject who is both the historical product and the site of certain practices, techniques, institutions and procedures of subjectification. ${ }^{41}$

Attending to the etymology of the term "observer" - meaning "to conform one's action and to comply with" - Crary describes the observer as "one who sees within a prescribed set of possibilities, one who is embedded in a system of conventions and limitations. ${ }^{142}$ The materialization of vision, making vision, as a site of practices and techniques, "visible," is one of the primary concerns of this study. My concern is revealing the contemporary machinic observer as a primary observing subject. Joining Johnston's understanding of the term "machinic" as an assemblage with Crary's "observer," we can begin to explore these concepts as they manifest themselves in our

41 Jonathan Crary, Techniques of the Observer: On Vision and Modernity in the Nineteenth Century (Cambridge, MA: MIT Press, 1990), 5 (Italics in original).

42 Ibid., 6. 
contemporary world, in the empirical material of AFR technology. In pursuing this concern, it is possible to reveal, in contemporary machine vision practices, certain historical continuities and discontinuities in the organization of vision. I seek to understand the materialization of the machinic observer's "system of conventions and limitations." Crary's focus on the organization and construction of vision as it is materialized through the human observer anticipates an organization of vision that is fully realized through the automated processes of AFR technology. His study reveals how vision has been constructed historically through the organizations of technology, society and science.

The theorists mentioned here so far have all contributed to a theoretical approach in how to understand forms of seeing that are machinic - as an assemblage, as codified, as industrialized and historically constructed. This provides a broader context within which to approach AFR as a technology in which the sense perception of vision gets enveloped by an automated process of recognition. In understanding AFR technology as one instance of a machinic observer, we can begin to formulate an account of how vision can be codified in a specific operation and within the conventions of "recognition." Yet, as I have explained, this codification is not without a history; it is, rather, embedded with historical and cultural practices of seeing.

In what follows, I use the phrase "a way of seeing" when referring to AFR technology as a way of making clear that these processes are embedded in cultural and historical contexts. This phrase is a direct reference to the work of John Berger, who seeks to understand the situated perspective of the observer in relation to works in art history. He mentions an especially central dynamic (albeit in a different context) between seeing and knowledge, which I argue underlies a central dynamic of machinic vision. He begins his book Ways of Seeing with the simple yet bold statement: "Seeing comes before words [...] The relation between what we see and what we know is never settled. ${ }^{243}$ He describes a gap between knowing something and seeing it. This gap is also a space in which a fluidity of mean-

43 John Berger, Ways of Seeing (London: Penguin Books, 1972), 7. 
ing can exist - a fluidity of meaning that occurs before it can be cemented in the meeting of the two. As an example, Berger refers to the artist Henri Magritte, whose paintings conjure up this gap as dissonance, in the play between text and form. Seeing as recognition through machine vision, reverses this order whereby knowing comes before seeing. An algorithm must know a face before it can recognize it. Further, an image recognition algorithm must "know" the object before it can successfully perform an operation of recognition. This seemingly simple understanding of the order of things in vision prompts an important theoretical critique in the form of a question, one that underlies the analysis set out here: if a recognition algorithm must know the object before it can recognize it, how does that transform and limit its knowledge of the things it sees?

In addition to the theoretical framework concerning machine vision, this analysis is also informed and framed by an account of the body and of the imaging of the body as well as theories of biopolitics. I have drawn on the work of Allen Sekula and his study of the intersection of the body and the archive. In addition, the theory of biopolitics, specifically what it has to say about the body in relation to information and being made "intelligible," has also informed my understanding of the shift in knowledge production that occurs through the introduction of AFR technologies. As I have already established, biometric systems, as risk mitigation and security technologies, have specific desired outcomes, namely, to establish identity as clearly as possible in a geopolitical landscape of uncertainty and in the context of border control. The application of biometrics in these arenas submits the body to processes in which it gets translated into data, in the service of institutional ends. This makes it possible to inscribe the body with the institutional statuses of nationality and other terms and identities constructed outside the person's subjective experience of his or her body. AFR technologies, and biometrics in general, operate by both negating and legitimizing identity. What is sacrificed within this paradigm is the subjective experience of the body; one's own construction of self, grounded in the human sensorium, gets negated when confronted with an identity imposed from the outside. The identity and status associated with the biometric identity then has the operative func- 
tion of dividing an individual not only from other bodies but also from herself. Biometrics creates a situation where one's body can act as a witness against oneself. It involves a process of dividing the surface of the individual, if you will, from what is beneath the skin, in which the body, in becoming a docile body subjugated to governance, becomes a subject of the state through its transformation into an image of the self.

This process of subjecting the human body to political inscription recalls Michel Foucault and what he terms "dividing practices," in which an individual's identity becomes defined in relation to society, and becomes vulnerable to exclusion according to prescribed categories, such as the "mad" and the "sane."44 This division between the internal subjective experience of self and the external naming of an institutional identity becomes part of the mechanisms through which an individual is turned into a subject of governance. Foucault also describes two registers through which the subject becomes transformed into a "docile body" under state power and is both subject and subjugated to governance. ${ }^{45}$ The first register is the "anatomico-metaphysical body" or "intelligible" body, that is, the body as an object of knowledge, for example the body as the object of certain forms of measurement. ${ }^{46}$ The second is the "technico-political" or "useful" body that submits to and is used in regimes of discipline and correction in institutions such as the army, hospital, prison and school. Through the practice of biometrics, the scanned body inhabits both of these registers: biometrics provides a body that can be read and a body that is of use in virtue of its ability to produce information. This accords well with what Foucault says of dividing practices: when one is categorized by social and state norms, one experiences a divide either inside oneself or between oneself and others. Biometric practices, again, encompass both of

44 Michel Foucault, "The Subject and Power," Critical Inquiry 8, no. 4 (Summer, 1982):777-778.

45 Foucault references the work of Julien Offray de la Mettrie, L'homme machine, 1747.

46 Michel Foucault, Discipline and Punish (London: Penguin Books, 1991), 136. 
these registers, in that, where the body can act as witness against oneself in the service of the categorical identities and statuses of the state, there exists a divide inside oneself, dividing what is beneath the skin from what is on the surface of it. One's embodied experience of subjectivity and the self is transformed into an image and becomes subjected to the state. The identity and status associated with the biometric identity then function to divide the individual from others.

How a body can be read and of use through a practice of biometrics is further referenced in the analysis through the work of Irma van der Ploeg, in particular her analyses on the "informatization of the body." Van der Ploeg argues that biometrics sets forth a new body ontology and she discusses biometric practices in relation to the supposed dichotomy between information - as in the "Information Age" - and materiality. She explains that technologies such as biometrics, which blur the line between "bodily matter" and "bodily information," have transformative effects on the level of embodiment and that this is important because "embodiment is central to individuality and identity in a way that my social security number or car rental records are not." ${ }^{37}$ Van der Ploeg provides an important discussion of how biometrics traverses multiple boundaries of knowledge and simultaneously demands new definitions of bodily integrity. Her arguments provide further support for the claim that the implementation of biometric processes transform our understanding of the subject.

\section{Outline}

This dissertation is divided into two main parts. The first part is a close analysis of the AFR method of eigenface and a historicization of the processes involved with this method. The second part at-

47 Irma van der Ploeg, "Biometrics and the Body as Information: Normative Issues of the Socio-technical Coding of the Body," in Surveillance as Social Sorting: Privacy, Risk and Digital Discrimination, ed. David Lyon (London: Routledge, 2003), 70. 
tempts to move toward a reimagining of facial recognition technology and its technical processes through an examination of the work of contemporary artists whose pieces reveal possible alternatives to the logic of this technology. Taken together, these two parts constitute an analysis of the visuality of automated facial recognition.

The first part begins with an introductory chapter on AFR technology, briefly outlining the problems and challenges that have arisen in the course of its development. The discussion then moves to an overview of the eigenface method and its technical features, outlining three aspects of its recognition process: Principal Component Analysis (PCA), the eigenvector and the "face space." The eigenface method is then related to the historical practice of composite portraiture as developed by Francis Galton, and I discuss the use of statistics as a way of seeing. Galton's practice of finding statistically salient patterns in composite portraits in order to create images of "types" serves to open up an inquiry into reductive forms of knowledge production. Ludwig Wittgenstein, who himself produced a composite portrait inspired by Galton, is brought into this discussion as the source of an alternative approach to the composite that does not follow the statistical logic of perception put forward by Galton. Wittgenstein refers to the composite portrait in the course of his philosophical investigations of language; for Wittgenstein, the composite portrait is both a rendering of the overlap of similarities between forms and a depiction of the particularities, and it thus resists any theory of generality. He refers to the composite specifically in relation to his idea of family resemblance and his account of concept formation. As I show, his perceptual interest in the composite lies not in the static center of the image, where the average can be perceived, but rather in the outlying areas, where a perceptual movement between forms can be perceived. This approach is discussed further with reference to Wittgenstein's concepts of "aspect perception" and "aspect blindness." Wittgenstein presents an alternative aesthetic approach to the composite portrait, one that directly inverts Galton's approach and, by implication, the statistical logic that underlies the eigenface algorithm. In this, Wittgenstein opens up a perceptual space in the composite portrait that defies the singular output of recognition, and he in- 
stead argues for a perceptual clarity grounded in being able to see the fluidity of forms. I argue that, in relation to the contemporary context of ubiquitous facial recognition technology, this Wittgensteinian approach has both utopian and political implications.

The second part of the book focuses on artistic interventions. The artworks included in this study are understood as providing visualizations of the algorithmic process as well as of critical theoretical concepts. The work of artists provides further context to the application of contemporary facial recognition technologies, while also reconceptualizing and reimagining the use of such technologies in society. Working specifically through a visual vocabulary, artists are in a unique position to articulate how these technologies play a primary role in enculturing processes of perception and representation.

The second part draws on the analysis provided in the first. It begins with a survey of composite portraits in art, before introducing the work of Thomas Ruff, in particular his studies of identification portraiture and composite portraits in his series andere Portraits (1995). This examination of Ruff's andere Portraits looks into his appropriation of the Minolta Montage Unit, a photographic apparatus first utilized by police in Berlin in the 1970s to produce composite mugshots. Although Ruff's series does not directly deal with an algorithmic process, his work is relevant to my discussion because it problematizes the forms of representation in facial recognition practices. By taking portraits of portraits, he raises questions about the forms of representation found in identification photography and confronts the diffused relationships between archival practices and the production of subjects, citizenship and identity. As such, his work expresses some themes that are latent in the eigenface image, and it can inform our understanding of the structures of representation found in facial recognition systems. I also argue that his work may be understood as a contextualization and visualization of Wittgenstein's concept of aspect perception. I analyze individual images from the andere Portraits series in order to discuss Ruff's rendering of the fluidity of gender, which serves as a critique of a binary understanding of identity. 
The next chapter covers the work of Zach Blas, and particularly his piece Facial Weaponization Suite. With this, the discussion of artistic interventions moves into the more contemporary context of facial recognition algorithms, presenting a conceptual strategy of resistance to the algorithmic gaze. I discuss Blas's own theoretical writings, which complement his art production with references to Édouard Glissant's concept of "opacity." Blas advocates a right to a specifically "informational" opacity, that is, a right to not be seen, identified and archived. Blas's work may be understood as a sculptural form of the composite portrait, that is utilized as a mask in order to resist identification. Blas's work confronts the reductive practices of automated recognition through a strategy using an excess of facial forms. In this way, I argue, Blas's work inverts the algorithm's representational mechanisms to resist an operation of algorithmic recognition.

The third chapter in this part is a study of the work of Trevor Paglen. Paglen's artistic approach can be understood in terms of a notion of transparency. Rather than confronting the end gaze of facial recognition technologies, Paglen's work ventures into the processes of algorithmic recognition that underlie this gaze, taking, as its artistic material, the training sets used by image recognition algorithms. In this way, I argue Paglen utilizes the algorithmic process as a ready-made object that has become ubiquitous in contemporary society and modifies its output in order to produce artistic objects. My study of Paglen's work focuses on two of his pieces: Adversarially Evolved Hallucination (2017) and Eigenface (Even the Dead are Not Safe) (2017). In the latter, through the subjects that Paglen chooses for his portraits, the eigenface image is related both to discourses in contemporary art and to discussions in political philosophy. These two works are further explored in relation to the goal of reimagining the very structures and outcomes that define algorithmic perception and its expanding role in the production of knowledge in society.

The concluding chapter summarizes the main findings and recommendations of this study and highlights potential areas for further research. This chapter also discusses the overarching theme of the composite as an aesthetic form that structures knowledge pro- 
duction in the Information Age. In closing, I offer some thoughts about controversial uses of facial recognition technology that came to light during the final stages of writing this book. 

Part I: Aesthetics of an Algorithm 



\section{Chapter 2: Eigenface}

\section{Background: Eigenface in the Development of AFR Technology}

Cultural historian Wolfgang Schivelbusch reminds us that, "as technological methods recede in importance, they reappear as an object of study." It has been almost thirty years since the AFR method known as eigenface was first developed. Since its introduction, more sophisticated methods of AFR have been developed and have become more widely used. Yet the eigenface method remains important to consider when investigating the visuality of AFR. The eigenface method served as a basis for the development of other procedures that went on to expand the possibilities of AFR. Its success validated the use of facial recognition algorithms and spurred on the development of facial recognition technology. For example, the development of the eigenface method made possible the introduction of fisherfaces, a more refined method allowing for a more precise recognition process. The methods of facial recognition more often used today rely on deep neural networks and other feature-based methods. The Viola-Jones algorithm (2001), for instance, uses a method of Haar Cascade to detect objects through superimposition, training an algorithm to differentiate true positives from increasing variations of false negatives. The methods that have since developed do not produce an image or deploy an ab-

1 Wolfgang Schivelbusch, The Railway Journey: The Industrialization of Time and Space in the 19th Century (Berkeley, CA: The University of California Press, 1986), xiii. 
stract notion of "seeing" through an algorithmic process. Programmers argue that today's AFR algorithms do not remotely resemble the human capacity of vision.

The eigenface method was introduced after what has been termed the "AI Winter" of the 1980s, when pessimism surrounded the technology development sector and funding for new technology declined. Nevertheless, this decade also saw the first use of the term "biometrics" in the media and in public forums to describe automated recognition systems. This was the result of a growing awareness in the field of automated recognition technologies ${ }^{2}$ that led to a "Coming of Age" of technological development in the 1970s. In the 1990s, the decade in which eigenface was introduced, the first "Biometric Consortium" was held, organized by the research division of the US National Security Agency (NSA). ${ }^{3}$

The eigenface method was first developed as a fully automated biometric facial recognition system by two MIT scientists, Matthew Turk and Alex Pentland, who developed it in conjunction with Arbitron, ${ }^{4}$ a television ratings company, for the purpose of monitoring ratings. Working within this consumer-marketing context, their goal was, as they have stated, to "develop a computational model of face recognition that is fast, reasonably simple, and accurate in constrained environments such as an office or a household. ${ }^{5} \mathrm{It}$ was designed to be used in TV sets to determine which individuals within a household were watching TV at which times, feeding this information into consumer ratings for specific television programs. Essentially, eigenface was designed to be integrated into a kind of TV that watches you as you watch it. Given this context, the ideal situation for the algorithm to operate in is a real-time situation in which people are sedentary. Ideally, the faces to be recognized would all be positioned conveniently, that is, squarely in front of the TV screen, in a neutral, forward-facing pose. This pose is familiar from identification photographs, so it was not a great leap to imag-

2 Wayman, "Scientific Development of Biometrics," 266.

3 Ibid., 269.

4 Turk, "Over Twenty Years of Eigenface," 2.

5 Turk and Pentland, "Eigenfaces for Recognition," 71. 
ine that eigenface might potentially have uses outside of the context of TV ratings, particularly in areas involving the use of identification documents. The multi-faceted application of facial recognition methods can be understood as a bleeding through from the sphere of consumer interests to the socio-political arenas of risk and control.

At the time of its introduction in 1991, the eigenface method was considered one of the first facial recognition methods successfully to perform face detection and recognition in real time. Before eigenface, automated recognition methods had focused on "feature extraction," that is, the recognition of isolated features (also termed "landmarks") of the face, such as the eyes, nose and mouth, and the measurement of the distances between these features. The eigenface method departs from this earlier approach by relying on a representational mechanism that takes into account a holistic representation of the face rather than its isolated features. In doing so, the eigenface algorithm had a built-in capacity to detect faces, as well as to locate, track and classify a subject's face.

\section{Bledsoe: "The Model Method in Facial Recognition"}

The introduction of the eigenface method marked a shift in the development of AFR technology not only because its seemingly simple technique was successful but also because it performed recognition differently from the AFR systems that came before it (and from those that would come after). The eigenface method shifted the approach of AFR methods towards a holistic representation of the human face. To understand why eigenface was considered successful, it helps to understand the original problems and challenges to which this technology was developed as a response. The first attempts to codify and automate facial recognition in an operable process were documented in reports authored by the computer scientist Woodrow Wilson Bledsoe, considered one of the founders of artificial intelligence. Two of these reports were only recently made publicly available (in 2014). They had previously been classified, while references to these reports described them as being commis- 
sioned by an "unnamed intelligence agency." Alongside the recent discovery and publication of these reports, it has also emerged that they were funded by a CIA front organization, the King-Hurley Research Group. ${ }^{7}$

Figure 1: "Examples of photograph pairs used in the study,"

Woodrow W. Bledsoe, 1964.

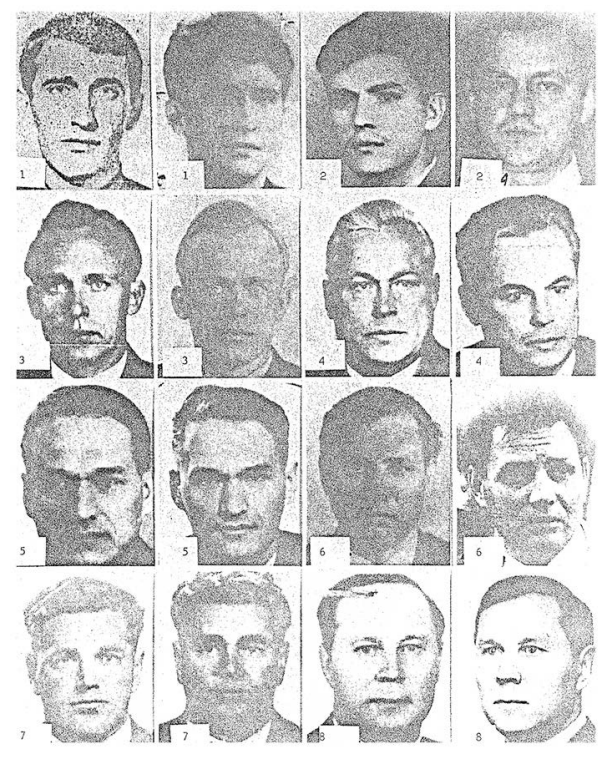

6 Michael Ballantyne, Robert S. Boyer, and Larry Hines, "Woody Bledsoe: His Life and Legacy," AI Magazine 17, no. I (Spring 1996): 7-20, https://doi. org/10.1609/aimag.v17i1.1207 and Wayman, "Scientific Development of Biometrics," 264.

7 The two Bledsoe reports were made publicly available thanks to the efforts of researcher Justin Lange, who, in 2013, as a master's student at the Interactive Telecommunications Program at New York University, was able to successfully retrieve them from the Dolph Briscoe Center for American History at the University of Texas. On the basis of his research into Bledsoe's reports, Lange concludes that the King Hurley Research Group, whose name is included in the title of one of these reports, was a front organization for the CIA. Lange's claim is corroborated by Christopher Robbins's book The Invisible Air Force: The Story of the CIA's Secret Airline (London: Macmillan, 1981). Thank you to artist Kyle McDonald for information about this backstory and for connecting me with Justin Lange. 
Thus, AFR technology seems to have originated as a mechanism for surveillance and intelligence accumulation in the context of national security operations, specifically under auspices of the research arm of the CIA.

Figure 2: "Double exposure shows that the two different subjects are surprisingly similar on a point by point basis," Woodrow W. Bledsoe, 1964.
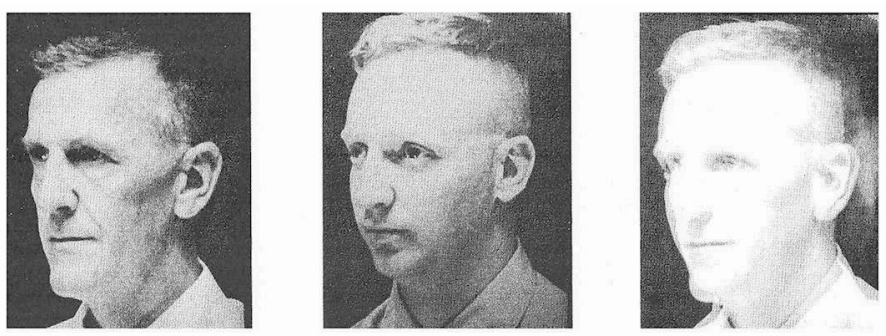

Figure 3: "Double exposure shows that the two poses of the same subject have very little in common when considered on a point by point basis," Woodrow W. Bledsoe, 1964.
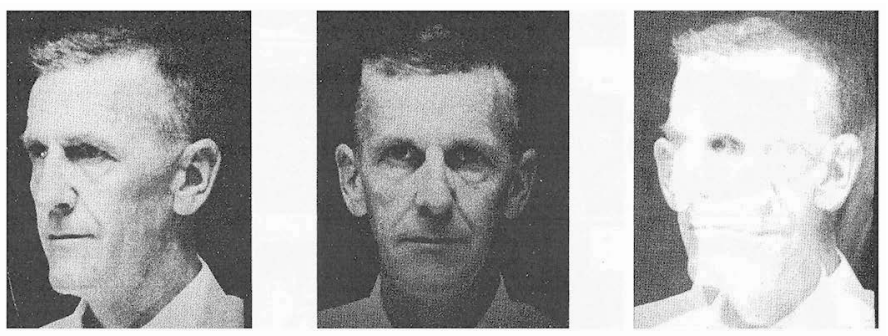

There are many fascinating details in these original reports of the pioneering and systematic attempt to automate the recognition of the face, but the most relevant to this discussion is Bledsoe's account of the problems and challenges of the task and how he chooses to visualize these. In Bledsoe's report, "The Model Method in Facial Recognition," Bledsoe notes that "one of the most challenging areas of pattern recognition is the identification of human 
photographs by machine." ${ }^{8} \mathrm{He}$ goes on to describe the difficulties of recognition on the basis of facial photographs, given the variations in age, expression, the angle and rotation of the face and in the direction and intensity of light hitting the face. This original account of the challenges faced by automated recognition is still mentioned in scientific papers published on AFR technology today. As part of this report, Bledsoe included a collection of training images, a dataset that he drew upon (figure 1). It is still not known where these original training images originated from, but they are all of white males of various ages. ${ }^{9}$

Bledsoe uses the method of double exposure to visualize some of these challenges confronting automated recognition. He pairs two portraits together that differ in the direction of facial rotation and superimposing one over the other to make clear the differences between a reference image and a capture image. Figure 2 is an example of this. It presents the facial faces of two men of different ages, yet these images exhibit the same direction of lighting and the same head rotation. Once superimposed, the faces merge. According to Bledsoe's caption, "two different subjects are surprisingly similar on a point-by-point basis. ${ }^{10}$ In a contrasting series of images, Bledsoe presents two images of the same man, now with different lighting direction and head rotation (figure 3). Here, he presents a double-exposure image depicting a jumble of ears, eyes and hair. Bledsoe describes how the two images of the same subject have very little in common. Bledsoe's exercise in superimposed photographic depiction conveys the central problem of similarity and difference in AFR technology, and thus the potential for false positives and false negatives. Bledsoe's early visual experiments with the overlaying of facial images foreshadow the eventual solution found in the eigenface method. The method of statistical pattern recognition

8 Woodrow Wilson Bledsoe, "The Model Method in Facial Recognition," Technical Report PRI 15 (Palo Alto, CA: Panoramic Research, Inc., 1964), 2.

9 Based on the demographics, Lange speculates that these portraits are from a criminal database. Bledsoe himself cites the work of Alphonse Bertillon and Cesare Lombroso on the "criminal man."

10 Bledsoe "The Model Method," 7. 
used in eigenface essentially utilizes these differences and similarities and encodes them. This early production of a binary composite reveals the kinds of challenges faced in the initial stages of the development of AFR technology. Yet Bledsoe's composites also anticipate the eigenface approach, which utilizes the representation of similarity and difference through superimposition and transforms this into a mechanism of successful recognition.

\section{Representational Mechanisms and the Machinic Observer}

The inspiration for the eigenface algorithm was the thought that it might be possible for algorithmic processes to mimic the processes of human recognition. This was expressed through a shift in the algorithmic modeling of the face from a concentration on its isolated features to a holistic representation of the face. Turk and Pentland were working at the intersection between physiology, information theory and the psychology of face recognition. They argued that human face recognition does not occur through the perception of individual facial features and the relationships between them, as previous algorithmic research had suggested. They stated: "individual features and their immediate relationships comprise an insufficient representation to account for the performance of adult human face identification." Instead, Turk and Pentland set out to build an algorithmic facial recognition technique that could produce a holistic representation of the face. This shift is important to consider. Algorithms depend on some form of representation and reproduction of the face. The form and design of this representation in the algorithm provides a foundation that allows the AFR technology to learn to recognize a human face. Marcin Miłkowski has defined the representational mechanism in computational learning processes as having three capacities: "it can refer to a target of representation, it can identify information about the target that is relevant for its own interests and goals, and it can evaluate the value of the infor-

11 Turk and Pentland, "Eigenfaces for Recognition," 72. 
mation based on environmental feedback. ${ }^{n 2}$ Here I use the term "representational mechanism" to refer to a part of the AFR process that is responsible for identifying information relating to a face that is relevant to a process of recognition. Eigenface relies on the use of a statistical method of pattern recognition as a representational mechanism. It is this representational mechanism which is depicted in the eigenface image.

Figure 4: "Sample face on top and its caricature below it." Sirovich, Kirby, 1987.
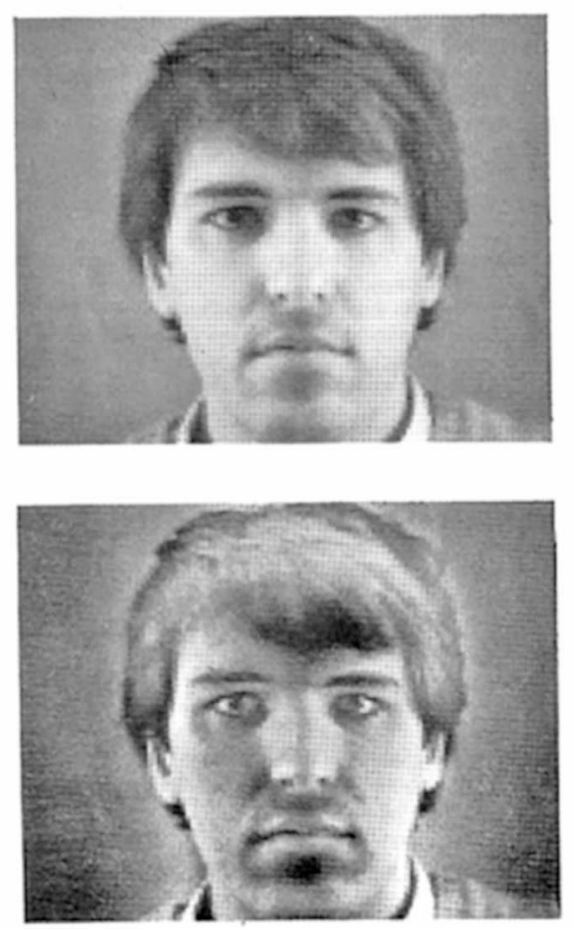

In order to produce the primary representational mechanism for eigenface, Turk and Pentland drew on the work of two scholars of applied mathematics at Brown University, Lawrence Sirovich and

12 Marcin Miłkowski, Explaining the Computational Mind (Cambridge, MA: MIT Press, 2013), 156. 
Michael Kirby. In 1987, Sirovich and Kirby published a paper on the use of a statistical method of pattern recognition called Principal Component Analysis (PCA), which they applied to facial images in order to produce what they called "eigenpictures."13 In their paper, Sirovich and Kirby speculate about how humans perform the function of recognizing faces and note how adept humans are at this complex task: humans can recognize an almost infinite number of different faces. They propose that humans are able to recognize so many faces because we engage in a process of deduction in relation to facial characteristics; that is, we recognize the characteristics of a face that depart from a kind of characteristic mean. They additionally propose a mathematical translation of this process, applied to multiple facial images, as a possible model for how humans recognize faces.$^{14}$ For example, at one point they refer in the text to photographs of two faces (figure 4), with one photograph having had this mathematical translation of the deduction process (referred to as the Fourier method) applied to it, a photo they refer to as a "caricature," and the other without having had this reduction applied to it. The photographs, they say, appear "virtually the same to us."15 The original (on top) is a still from a video, whereas the other, having undergone a mathematical reduction of pattern recognition, is its transformation into a computational image. When looking at these two facial images, I see a significant difference between the "caricature" and the original; the caricature looks as though it has undergone what is referred to in photography as a solarization process, whereby a tonal inversion occurs through the developing process of a photographic image. In the caricature, the mid-tones and shadow areas become darker while any highlighted areas, in contrast, become brighter. It is as if the tonal spectrum of the image has been compressed and the differences between tones are made more extreme. Sirovich and Kirby, however, conclude that, since the images

13 Sirovich and Kirby, "Low-dimensional Procedure," 521. Although Sirovich and Kirby were the first to apply PCA to facial images, Turk and Pentland were the first to design an automated recognition system utilizing PCA.

14 Ibid., 519.

15 Ibid., 523. 
appear the same to them, they provide evidence that "our own visual apparatus does a similar subtraction." ${ }^{\text {16 }}$ In other words, our own perceptual processes of recognition most likely involve a process of some form of deduction.

In their recorded observations and conclusions it is possible to detect an interesting and altogether separate confrontation that occurs in these initial eigenpictures, and that is a confrontation between human and machine perception. It is exemplified by Sirovich and Kirby's reference to "our own visual apparatus." For the mathematical caricature, the video still and our own processes of perception all represent intersections of perceptual relationships between multiple apparatuses - photographic, video, computational and bare human perception - that inform an exercise in recognition. With their speculation about the human ability to perceive faces and the possibility of expressing this capacity in these abstract and mathematically based caricatures, Sirovich and Kirby stray into the realm of what John Johnston has described as "machinic vision," that is, "not only an environment of interacting machine and human-machine systems but a field of decoded perceptions that, whether or not produced by or issuing from these machines, assume their full intelligibility only in relation to them." ${ }^{17}$ Two key movements that Johnston outlines in relation to machinic vision are a deterritorialization and a reterritorialization of vision. ${ }^{18}$ The former occurs when visual perception is freed from the person that is doing the seeing, and the latter occurs when that seeing is "recoded," that is, recontextualized and expressed in a new form so as to produce new meaning. Sirovich and Kirby's speculation may be understood along these lines: the caricature is an expression of vision, in a process of recognition, freed from human cognition.

\footnotetext{
16 Ibid.

17 Johnston, "Machinic Vision," 27.
18 Ibid., 28. Here Johnston is drawing on Gilles Deleuze and Félix Guattari's use of the term "machinic."


Figure 5: AT\&T Laboratories, Eigenfaces offaces from the ORL face database.
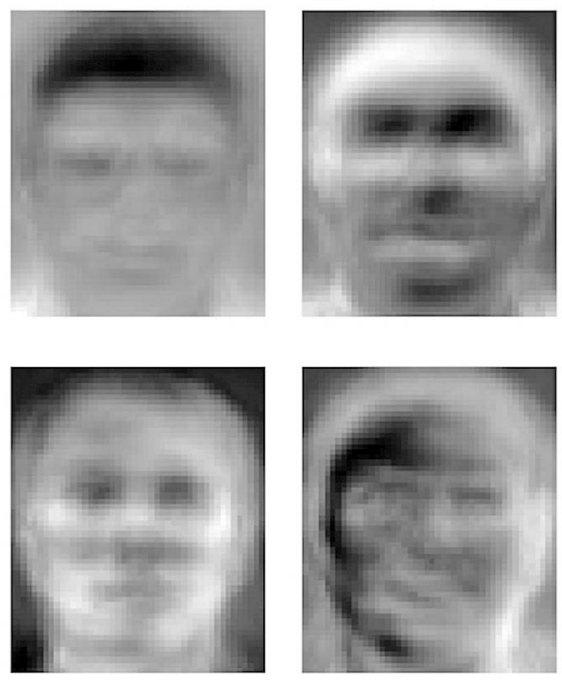

Sirovich and Kirby pay close attention to their own visual perception of these caricatures. They believe that the conditions of recognition can be revealed through them. In this way, the caricatures also reveal the conditions of human recognition. What is also important to consider is that, for Sirovich and Kirby, the caricature makes visible the human recognition process; in other words, it makes it possible to see how we see. In this way, it reveals an "observer," in the sense of the term Jonathan Crary explains in his study of the historical construction of vision: "one who sees within a prescribed set of possibilities, one who is embedded in a system of conventions and limitations. ${ }^{19}$ Crary argues that it is only through the observer that vision, in history, is able to "materialise, to become itself visible."20 These early experiments with eigenpictures express an early assemblage between human cognitive processes of recognition and a machinic translation of that recognition. In the caricature, a kind

19 Crary, Techniques of the Observer, 6.

20 Ibid., 5. 
of "machinic observer" is revealed, and we can begin to see how vision can be codified within a specific operation and within the conventions of an operation of recognition. In other words, Sirovich and Kirby's early eigenpictures and caricatures give a visual form to the conditions of recognition.

\section{Three Aspects of Eigenface}

The German prefix eigen- means "inherent, own, individual, peculiar, specific, and characteristic." ${ }^{21}$ As this suggests, the facial recognition method is supposed to be an algorithmic ability to distinguish what is characteristic of an individual's face in order for the algorithm to determine the individual's identity. The eigenface algorithm is designed to do just this. Eigenface is based on the premise that the most relevant information about an individual face has to do with the ways it is different from another. Eigenface has been successful in demonstrating an ability both to detect faces and to encode the differences between multiple faces. As Turk and Pentland state, "A simple approach to extracting the information [...] is to somehow capture the variation in a collection of face images [...] and use this information to encode and compare individual face images." 22 Indeed, the primary difficulty in developing a successful AFR system is, as Bledsoe had earlier realized, that human faces vary endlessly in appearance. The eigenface method takes this difficulty and transforms it into a recognitive capacity through a tool of differentiation. In this way, variation is utilized in encoding an individual face. Yet, far from distinguishing particular characteristics, the eigenface image depicts a very different process: an erasure of all individual facial particularities (figure 5). All that is specific and particular to a human face seems to dissolve in a blur. This paradox between the method and the image harbors a contradiction in the modalities of recognition between algorithm and human. To further elaborate on

21 "Eigen," Wiktionary, last modified April 7, 2019, https://en.wiktionary.org/ wiki/eigen\#German.

22 Turk and Pentland, "Eigenfaces for Recognition," 73. 
the mode of recognition by eigenface, I will describe briefly here three key technical aspects of the eigenface process that produce this image and that constitute its representational mechanism and operation of recognition. These three aspects are Principal Component Analysis, the eigenvector and the face space.

Principal Component Analysis is a statistical procedure that has primarily been used as a classification tool and as way of producing predictive models based on a statistical method of mean centering. In mathematical terms, PCA treats each facial image as a point or a vector on a grid with a high-dimensional space allowing for high degrees of variation. This high-dimensional coordinate space can be understood as Cartesian space gone digital. Each collected facial image in the training set is translated into a unit of measurement, or a weight, within this virtual space. Averages are calculated from the different weights of facial images. Each average takes into account all the possible variations of each weight. The averaged or mean face is described as "the center of gravity for all the faces combined." ${ }^{23}$ This averaged face delimits the highest degrees of variation, that is, the farthest directions of deviation from the average that exist between the collected facial images. Turk and Pentland explain that "any collection of face images can be approximately reconstructed by storing a small collection of weights for each face."24 The PCA procedure calculates a mean by averaging the value of each pixel across the face images. PCA is able to extract the principal components, or the primary differences, between multiple faces and encode this variation. Eigenface programmers describe this as revealing the internal structure of the data. Sirovich and Kirby state: "It seems reasonable to assume that an efficient procedure for recognizing and storing pictures concentrates on departures from the mean. With this in mind, the deviation or departure from the mean is defined. ${ }^{25}$ What they describe is a way of defining the char-

23 Jeremy Kun, "Eigenfaces, for Facial Recognition," Math Programming (blog), July 27, 2011, https://jeremykun.com/2011/07/27/eigenfaces/.

24 Turk and Pentland, "Eigenfaces for Recognition," 73.

25 Sirovich and Kirby, "Low-dimensional Procedure," 519. 
acteristics of faces through the differences between faces. The mean, therefore, becomes a base from which to designate a difference.

As in many facial recognition algorithms, the application of PCA begins with a training set produced from multiple facial images. In 1986, Sirovich and Kirby were the first to experiment with building a training set of faces on which to apply the method of PCA. They report taking still analogue shots of video recordings. ${ }^{26}$ Their first test group of faces came from their immediate environment, the relatively homogenous population of "the undergraduate male population" that dominated the mathematics department at Brown University, a group they describe as made up of "smooth-skinned caucasian males." They recorded the faces for the training set using video, on top of which they overlaid a cross-hair aligned vertically with the midline of the face and horizontally with the pupils. They were able to adjust the depth of field of each video still so that the width of each face could be equalized. These images were then digitized and turned into gray-scale pictures through an image processor. The result of PCA is a mean of all the faces of the training set and is depicted in an image in Sirovich and Kirby's paper (figure 6).

Figure 6: Sirovich, Kirby, "Average face based on an ensemble of 115 faces." 1987.

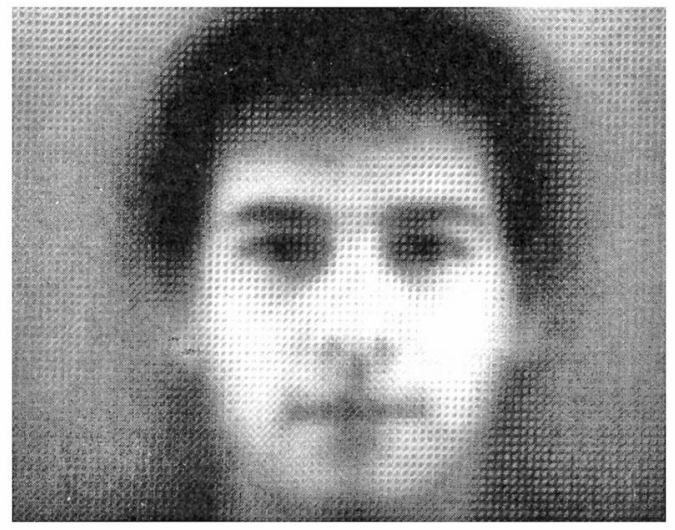

26 Ibid., 522. 
These initial experiments with training sets and the application of PCA are interesting to examine because the average male face was constructed from a training set collected from the exclusively white, male population of the applied mathematics department at Brown University. In choosing faces whose characteristics were closer in similarity, it raised the threshold of success for recognition. Sirovich and Kirby describe how they purposefully chose faces that were similar to each other in order to produce the best outcome. ${ }^{27}$ Higher rates of positive recognition are thus dependent on a smaller degree of difference between the faces included within the modeling of the average face. ${ }^{28}$ Similarities between the physical characteristics of faces is part of the logic of recognition. Even at this stage, the building a viable training set, there is already a kind of reduction being applied, a normative categorization of faces according to characteristics of gender and race.

There is another technical reduction process that occurs at this initial stage. The facial images that make up the training sets are converted to gray-scale (if originally in color) and to low pixel resolution. This reduction is indicative of perceptual conditions that contrast with those of human processes of recognition. The conversion to low resolution (usually at $200 \times 180$ pixels) and to gray-scale, that is, to values of light and dark, reduces the amount of visual information available to the human eye. Yet, for the algorithm, this reduction provides clarity by way of "simplifying" the images - to use the vernacular of digital post-production terminology - meaning that it leaves only the information necessary for the operation and gets rid of the rest. Reduction by way of gray-scale and low resolution eliminates the obscurity or extra information that detracts from the ingredients the algorithm deems important, that is, what can be measured and calculated through pixel values.

The reduction of the information in the image provides a better "palette" for pattern recognition to take place. These initial processes of reduction, both in the format of the images and in the choice

27 Ibid., 524 .

28 This method made possible improvements to the eigenface algorithm and the development of the method of "fisherfaces." 
of demographic from which the faces are drawn, are part of constructing the representational mechanism of the algorithm; that is, they help shape what is deemed salient and relevant to an operation of recognition. In applying statistical processes of reduction, PCA performs a kind of transformation of facial variations into a "working object." As Lorraine Daston and Peter Galison explain, a "working object" as, "any manageable, communal representative of the sector of nature under investigation" such as atlas images and type specimens, that at times replace the natural specimens they represent." ${ }^{29}$ Organic forms produce endless variation and are unable to "cooperate" in generalizations and comparisons. In contrast a "working object" provides a common object inquiry. In describing scientific atlases as a "working object," Daston and Galison explain how it served to "teach how to see the essential and overlook the incidental, which objects are typical and which are anomalous, what the range and limits of variability in nature are." ${ }^{30}$ The principle of the "working object" was based on allowing for collective scientific inquiry to occur through the standardization of natural forms. As Daston and Galison show, the creation of working objects was central to the work of scientific inquiry and the classification of natural phenomena. PCA performs the task of transforming faces and facial variations into a manageable and workable object by defining the range of facial variations in an operation of recognition. The statistical method of PCA is able to merge multiple natural forms of faces into a single conglomerate. As with the working object, PCA is able to refine the facial variations and envelop them within a readable (at least by a machine) working object of the averaged face, transforming the variations into a manageable form. In this way, the statistical process of PCA functions like a scientific atlas of the algorithm to train it to see the essential aspects of the human face, found in the average but also to see the "incidental" or rather the deviations from the average as a method of recognition.

29 Lorraine Daston and Peter Galison, Objectivity (Cambridge: Zone Books, 2007), 19.

30 Ibid., 26. 
Training sets are the data banks and, as such, the source of knowledge for an algorithm. They provide knowledge for the algorithm on what it is allowed to see and recognize. In this way, for an algorithm, the training set is the link between knowing and seeing. The training set is what enables the algorithm to know certain faces and thus to recognize them. The early examples of images of average faces produced by Sirovich and Kirby's training sets reveal a bias toward white men as a primary and normative category of recognition. Although the training sets used in their research were part of an experiment with eigenface and not examples of the actual application of AFR technology, the demographic of the people in the images corresponds with that of the people in the training sets in the initial experiments with AFR technology conducted by Bledsoe. As such, there is a history of the training sets used in the development of automated processes of recognition predominantly involving the figure of the white male. In their analysis of facial recognition systems, Lucas D. Introna and David Wood remark that such reductions are where bias can be located in the algorithmic process. ${ }^{31}$ Although they do not scrutinize specific AFR methods in great detail, based on their examination of training databases they speculate that, through the reductive process of both image-based and feature-based algorithms, minorities are most likely to deviate from statistical averages that result from facial recognition systems. In analyzing the problematics of reduction, they conclude that minorities of Asian and African-American descent are the easiest to recognize in virtue of this deviation from the mean. ${ }^{32}$ This, they argue, contradicts the claims of suppliers and security analysts in the biometric industry about the neutrality of AFR systems. ${ }^{33}$ In light of Introna and Wood's findings and the "average faces" presented in the papers on the development of eigenface, we may conclude that representation in facial recognition systems has been dominated by the white male, presenting all other demographics as deviations from the norm.

\footnotetext{
31 Introna and Wood, "Picturing Algorithmic Surveillance," 186.

32 Ibid., 190.

33 Ibid., 191.
} 
The application of PCA to training set images creates "eigenvectors," that is, mathematical objects that display the degree of variability or deviation between facial characteristics and an average. Each eigenvector represents the greatest degree by which the facial images may vary - i.e., the highest eigenvalues. Multiple eigenvectors result from applying PCA to training sets, creating a mechanism to classify unknown faces based on their deviation from these eigenvectors. The eigenvector is a virtual model of "known" faces and serves as a reference point for the classification of unknown faces. Like a map, an eigenvector stands as a kind of idealized mod$\mathrm{el}$; it acts as a primary referent on the basis of which the algorithm is able to measure the distance or variation between it and an unknown face. As a virtual model, the eigenvector is a form of representation that transforms the pictorial, individual representations of known faces into a geometrical space defined by facial measurements. In this way the eigenvector comes to represent faces based solely on their relationships to other faces. The eigenvector is a representation of the differences and similarities between faces and in this way functions as a unit of facial measurement.

It is only when an eigenvector is displayed to meat eyes, that is, to human vision, that it is referred to as an eigenface. The greater the variation of an eigenvector, the more blurred the eigenface appears. Programmers have referred to eigenvectors as "ghost faces"34 because of their phantom-like appearance. These programmers are describing the form of these eigenvectors, which is characterized by the multiplicity that is inherent in the statistical process. The programmers' reference to ghost faces evokes a notion of imagery put forward by W. J. T. Mitchell, who describes a type of imagery that is perceptual and occupying, "a kind of border region where physiologists, neurologists, psychologists, art historians, and students of optics find themselves collaborating with philosophers

34 Müge Çarikçi and Figen Özen, "A Face Recognition System Based on Eigenfaces Method," Procedia Technology 1 (2012): 122, https://doi.org/10.1016/j. protcy.2012.02.023. [118-123] 
and literary critics." ${ }^{35} \mathrm{He}$ further describes this imagery as playing the role of fantasmata and as existing as "revived versions of those impressions called up by the imagination in the absence of the objects that originally stimulated them." The eigenface image can be understood as this type of fantasmatic image. Indeed, one of the primary aesthetic features of the eigenface image is the absence of the individual face, which disappears in the midst of its conglomerate form. Instead, the facial appearances in the eigenface image function symbolically to construct the virtual facial model, which acts as the central referent in the recognition process.

The collection of eigenvectors create a subspace referred to as the "face space." Eigenface developers have described the face space as a virtual subspace that is defined and framed by the measured distances between a collection of eigenvectors. The concept of a face space derived from the field of psychology in interpreting how faces are processed by human recognition. The face space is originally defined as a, "multidimensional psychological space, in which faces can be represented according to their perceived properties" and with the, "assumption...that faces (or concepts) could be represented as a collection of interchangeable parts. ${ }^{36}$ The face space in the eigenface algorithm is defined by the range of variability of these "interchangeable parts." Conceptually, the face space spans all possible variations of faces. Turk explains that any kind of:

image deviations (whether due to image noise or other factors, such as illumination, pose, expression, occlusions, etc.) push an image away from the space, and the distance from face space can be used to determine how likely an image is to be a face in the first place, thus providing a built-in mechanism for face detection. ${ }^{37}$

35 W J. T. Mitchell, Iconology: Image, Text, Ideology. Chicago: University of Chicago Press, 1986, 10.

36 Tim Valentine, Michael B. Lewis, and Peter J. Hills, "Face-Space: A Unifying Concept in Face Recognition Research," Quarterly Journal of Experimental Psychology 69, no. 10 (2016): 1996-2019, https://doi.org/10.1080/1747 0218.2014.990392.

37 Turk, "Over Twenty Years of Eigenface," 2-3 (italics in original). 
Turk's description thus identifies the face space as the source of facial detection in the algorithm. The face space operates as a virtual data bank, storing an algorithm's knowledge, enveloping all the variations of possible faces that the eigenface algorithm can conceivably recognize. The collection of eigenvectors with highest eigenvalues, that is, highest measured variations, is used as a referential source for algorithmic knowledge. In this way, the face space is like a virtual filing cabinet - the primary bureaucratic mechanism of identification in criminology. But instead of a filing cabinet storing individual records, the face space collects statistical averages or eigenvectors to serve in the process of recognition and verification.

The actual recognition process in eigenface involves projecting the captured image of the individual who needs to be identified on to the face space. The image is compared with the face space by calculating the Euclidian distances between the eigenvectors and the captured image. If there is a small distance between the capture and the eigenface, there may be a match. The distances between them are then expressed in numerical values and a data set is created. This data set then represents a person's identity and is entered into a database. An individual is classified within a biometric database not through their image but rather through numerical code. If there are large distances between the capture and the eigenvectors within the face space, then there is no match. If there is no match, the captured face can be incorporated into the algorithm, adding a new variation within the eigenvector. Turk and Pentland expanded on Sirovich and Kirby's use of the PCA method by incorporating this machine-learning technique within the eigenface method. They state: "The concept of face space allows the ability to learn and subsequently recognize new faces in an unsupervised manner." 38 Turk and Pentland describe the process by which the detection of unrecognized faces builds new patterns in the algorithm:

When an image is insufficiently close to face space but is not classified as one of the familiar faces, it is initially labeled as "unknown."

38 Turk and Pentland, "Eigenfaces for Recognition," 79. 
The computer stores the pattern vector and the corresponding unknown image. If a collection of "unknown" pattern vectors cluster in the pattern spaces the presence of a new but unidentified face is postulated. ${ }^{39}$

The statistical average or eigenvector defines the recognition procedure and is the form of measurement in relation to which a face is or is not recognized. In machine learning, the algorithm is designed to continue developing over time as facial variations are continuously added and learned.

Figure 7: Vincent Scheib, "Eigenfaces of UNC".

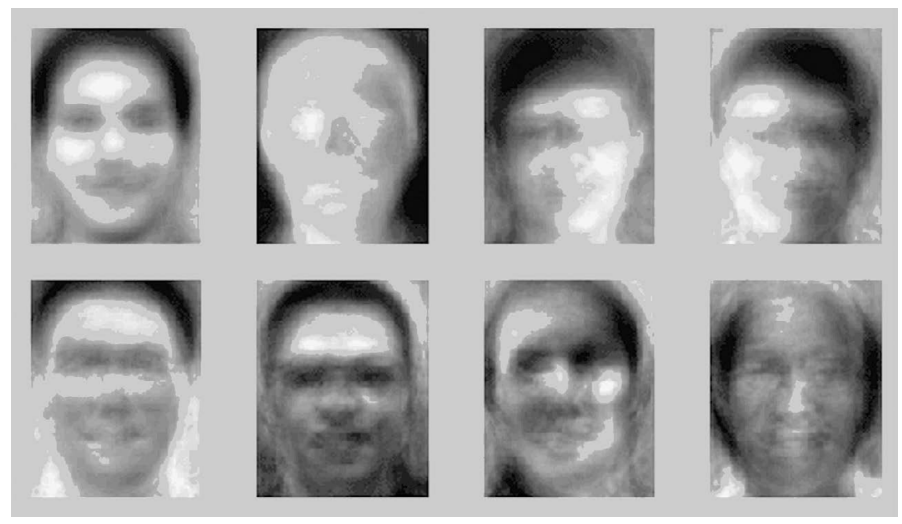

Face spaces provide visual sources of information about the eigenface process. As collections of eigenvectors, face spaces depict a wide spectrum of measured distances of similarity and difference. I present three face spaces here in order to illustrate the kinds of variation they exhibit, as well as the issues and problems that arise in the construction of these spaces. By presenting eigenvectors side by side, face spaces can illustrate specific aspects of variation in these eigenvectors. The face space in figure 7 is typical of the face spaces created by programmers working with eigenface. One thing that we can see clearly in this face space is the variation between the dif-

39 Ibid. 
ferent eigenvectors. ${ }^{40}$ The eigenvector at the top left has the highest eigenvalues, representing the average of all the 201 faces within the training group. The eigenvector immediately to the right captures the overall brightness of the face in the picture. The two following eigenvectors to the right capture the direction from which the face is illuminated. The eigenvectors on the bottom row capture variations in face shape. As the programmer states, the rest of the eigenvectors, of which there were 201, capture more subtle details.

Figure 8: Alexandra Feldman, "Face Recognition: Final Project CS 129, Spring 2011," Computer Science at Brown University
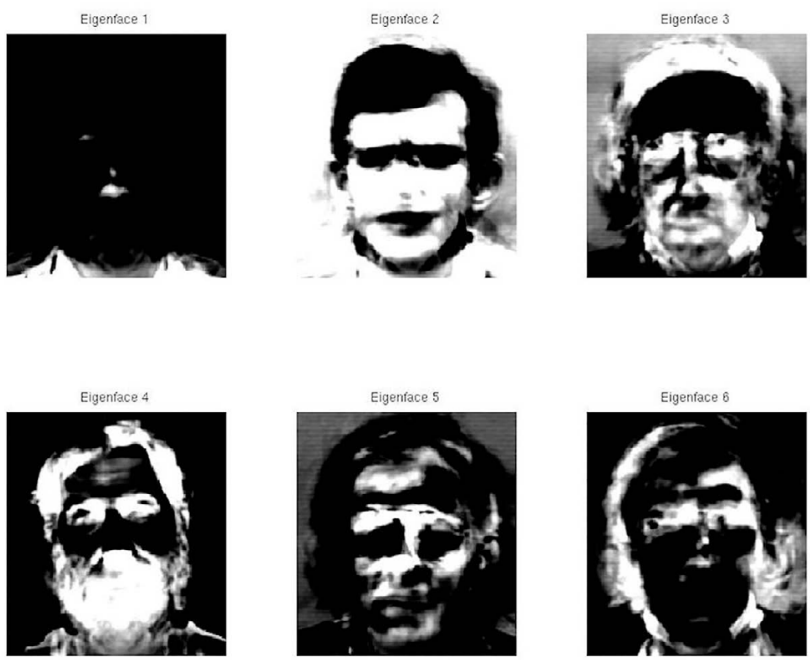

The greater the variation of an eigenvector, the more blurred it appears. For example, figure 8 is a face space created by a computer science student at Brown University. ${ }^{41}$ This face space stood out to

40 Vincent Scheib, "Eigenfaces of UNC," accessed April 25, 2015, http://www. scheib.net/school/uncfaces/index.html.

41 Alexandra Feldman, "Face Recognition: Final Project CS 129, Spring 2011," Computer Science at Brown University, accessed April 25, 2015, http:// cs.brown.edu/courses/csci1290/2011/results/final/amfi/. 
me from other face spaces because each individual eigenvector is constructed through extreme contrasts of light and shadow, creating a grouping that is aesthetically characterized by a painterly effect, where each conglomerate facial feature resembles a brush stroke. Rather than the usual blur, the facial features are defined through blunt markings of light and shadow. The eyes of the figure in the upper-middle eigenvector are blacked out, like two large ink spots, while the eigenvector in the lower left sports a glowing beard. From a programming perspective the student who constructed this face space was considered to have made a gross error (and was required to fix the contrast levels in her algorithm), ${ }^{42}$ its tonal extremities reveal an aspect of the process by which face spaces are built. Increasing the contrast levels so dramatically causes the feature similarities within each eigenvector to become more distinct - so much so that these distinctions begin to merge into each other and construct other (facial) forms out of the composited similarities. The eigenvector in the upper-left corner is rendered almost completely black. This eigenvector has the highest eigenvalues in the face space. The higher value, which equates to a larger amount of information for machine perception, amounts, paradoxically, to the least visually coherent image for human vision.

Figure 9: Wonju Lee, Minkyu Cheon, Chang-ho Hyun, and Mignon Park, "Best Basis Selection Method Using Learning Weights for Face Recognition," Sensors (Basel) 13, no. 10 (October 2013)

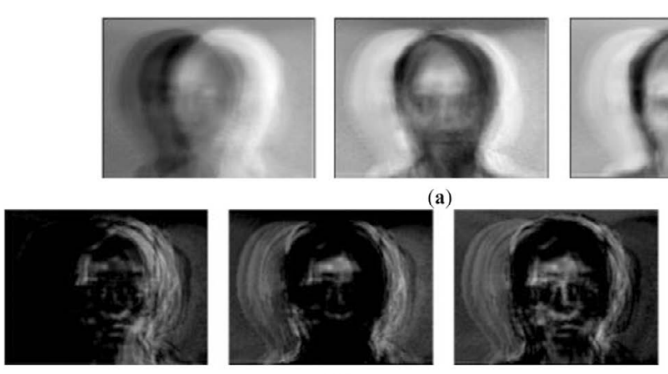

(b)

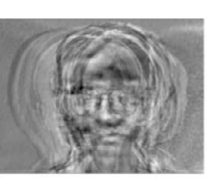

(c)

42 Ibid. 
Figure 9 is a face space included in an article written by four engineers who were experimenting with an alternative method for selecting eigenvectors for face spaces. In this method, the eigenvectors were selected not on the basis of their eigenvalues but rather through groupings of similar, closely related faces. ${ }^{43}$ The face space thus created highlights the extreme differential values produced by misaligned faces and is expressed through varying tones of illumination. The silhouette of differently positioned faces creates a halo effect. These "halos" consist of light and shadow that correspond to high eigenvalues. As in the previous face spaces, the eigenvalues descend in order, starting in the top-left corner. The students who created this face space argue that the eigenvectors in group (a), the three images on the top row, are "unimportant eigenfaces" because they do not "have discriminant information"44 that would allow the technology to perform an act of recognition. They argue that the faces in the training set should be cropped even closer so as not to include the illuminated edges within the eigenvalues of an eigenvector. Their rationale here is that the variation in illumination was found to have weakened the overall recognition process, and their aim was to find ways to more tightly define facial variation in order to allow for more precise techniques of recognition.

\section{The Eigenface Image}

I have outlined these three technical aspects, the PCA, the eigenvector and the face space, because they structure the ways in which the algorithm performs recognition. They also form the empirical basis for my own analysis of how the algorithm perceives. These three aspects provide routes into an understanding of the conditions of recognition in an AFR system. They explain how a face comes to be

43 Wonju Lee, Minkyu Cheon, Chang-ho Hyun, and Mignon Park, "Best Basis Selection Method Using Learning Weights for Face Recognition," Sensors (Basel) 13, no. 10 (October 2013): 12830-51, https://doi.org/10.3390/ s131012830.

44 Ibid., 12834. 
known and how information is produced and archived by the algorithm through successful operations of recognition. In order to understand these processes within the cultural and socio-political contexts in which they take place, I plan not to examine their more technical elements but instead to place them into a dialogue with ideas from the field of visual culture theory and to recognize them as enculturing an algorithmic way of seeing and medium of thought. What I mean by "medium of thought" here is that eigenface is not a static technical process; it is rather designed to learn new faces continuously and to incorporate them within its face space. The representational mechanism of PCA and the production of eigenvectors are the eigenface algorithm's means of knowledge accrual. The eventual operation of recognition produces knowledge of an individual's identity. In this way, the algorithmic way of seeing is intertwined with modes of contemporary knowledge production. These technical aspects thus reveal the ways in which the algorithm sees and knows. John Berger opens his seminal book Ways of Seeing by outlining the intimate relationship between seeing and knowing: "Seeing comes before words [...] The relation between what we see and what we know is never settled. ${ }^{345}$ Berger describes a productive and "always-present gap" between knowing and seeing that is fundamental to the experience of visual perception. ${ }^{46}$ In contrast, seeing by way of recognition through an automated algorithmic process reverses the order Berger describes. Knowledge comes before seeing. In this section, I examine this reversal by asking how it affects the subject being seen and by tracing the ways of seeing present in the aspects of the eigenface algorithm discussed above.

The production of the eigenface image marks a kind of pictorial turn, albeit a brief one, in the development of automated facial recognition technology. In this turn, images come to supplement equations in the operations of the algorithmic process. The eigenface image is a visualization of a statistical process. It depicts a statistical way of seeing in which the eigenvector, or the aggregate face, becomes the source of knowledge for the algorithm. Statistics, as a

45 Berger, Ways of Seeing, 7.

46 Ibid. 
way of seeing, is not new. In fact, statistics has been described as a tool of visualization, as a way of seeing on a scale beyond the capacity of human senses. In his critical genealogy of the term "information," media theorist John Durham Peters describes how statistics merged with an understanding of information in contemporary society. Peters notes that the etymological origins of the word lie in the German statistik, meaning the comparative (and competitive) study of states. Statistics arose as a tool of politics and state governance. Peters states, "The scale of the modern state presents its managers and citizens with a problem: it is out of sight and out of grasp. It must be made visible. ${ }^{377} \mathrm{He}$ goes on: "statistics arose as the study of something too large to be perceptible - states and their climates, their rates of birth, marriage, death, crime [...] [Statistics is] a set of techniques for making those processes visible and interpretable. ${ }^{n 8}$ As such, statistics is a visual tool that is inextricably bound up with its original context of use: state governance and control.

Peters highlights an analogy between the acquisition of knowledge and the body, describing statistics as the "empiricism of the state," whereby "the state becomes the knower, bureaucracy its senses, and statistics its information. ${ }^{349}$ When information comes to be understood in the form of statistics, the process of knowledge accumulation relocates from the site of the body to the site of the governing institution. Peters characterizes information produced through statistics simply as "knowledge with the human body taken out of it." ${ }^{50}$ Considering the use of the statistical method of PCA as the representational mechanism of eigenface, this suggests an ontological paradox. The eigenface method, and other AFR methods, are wholly reliant on statistical pattern recognition processes to produce information, but this information is constituted by and issues from the body itself. This presents us with a situation in which the physical phenomenon deemed invisible and ungraspable is none other than the body itself.

47 Peters, "Information," 14.

48 Ibid.

49 Ibid.

50 Ibid., 15. 
The foregoing discussion about statistics as a tool of vision raises the issue of the treatment of the body as information. Btihaj Ajana, a scholar working within the field of digital cultures, sets out an important approach to the understanding of the body as information with regard to biometric practices. Drawing on Eugene Thacker's concept of "biomedia," she argues for an understanding of the use of biometrics as "less as a tool and more as a process, less as an instrument and more as an act." ${ }^{151}$ Along these lines, Ajana argues that the result of these biometric processes and acts is that the body is rendered as "both the 'medium' (the means by which 'measurement' is performed) and the 'mediated' (the 'object' of measurement). ${ }^{52}$ Through the differential calculations of the eigenvector, the recognition process in the eigenface method realizes the convergence between these two roles that the body plays. Irma van der Ploeg claims that the treatment of the body as information in biometric practices introduces a new body ontology. She describes a process of the "informatization of the body," in which bodies are represented in digital code, which "construe[s] the body in terms of flows of information and communication patterns. ${ }^{153}$ She describes the body as a historical construction (much as Peters describes information) that is "implicated in a process of co-evolution with technology - information technologies, but also surgical, chemical and genetic and visualization techniques, and combinations of these. ${ }^{n 4}$ She describes how biometrics produces new forms of knowledge that transform our understanding not only of technology but of the body itself.

The eigenface image, as a visual artifact of the algorithmic process, allows us to investigate the visuality of the informatized body of AFR systems. The image presents us with the way faces are depicted, read and treated as information. The visualization of its patterns and form is a result of a statistical representational mechanism, PCA. Algorithms are often invisible, operating in a "black box" and leaving no trace of their processes of computation behind.

51 Ajana, Governing through Biometrics, 23 (italics in original).

52 Ibid.

53 Van der Ploeg, "Biometrics and the Body," 64.

54 Ibid. 
The algorithm does not need to produce pictures to understand its own process of recognition. The picture is for human eyes. The eigenface image serves as a training image, not for the algorithm but rather for us; it allows human eyes to see like algorithmic eyes. As a window into this process, the image answers one of the sociological criticisms of AFR systems, namely, that they lack reciprocity - its systems identify people without identifying with people. The eigenface image provides a form of visual reciprocity, depicting what and how the algorithm "sees." But observation of the eigenface image provides the very opposite of clarity concerning a person's identity. The eigenface image depicts a moment of stasis between the multiple inputs of data from the training set and the singular output of recognition. The images compiled in the eigenface training sets offer a multitude of possibilities and a wealth of variation, while the operation of recognition reduces the output to one possible match. Positioned at this in-between phase of the algorithm, the eigenface image not only presents a statistical process but also preserves a moment at which multiple possibilities remain open.

Turk has explained that he and Pentland designed the production of the eigenface image as part of the algorithm because they "wanted to keep clear of the 'black box' approach of [...] neural networks [...] in order to have a better ability to understand and debug the method." The eigenface image is part of the recognition operation; it is really information and not an image in the traditional sense. It is an example of what the artist Harun Farocki has termed an "operational image," "the aesthetics of which were not intended [...] instead of representing the objects in the world, these images are doing things in the world, they are part of a process [...] they are information and not really images." ${ }^{55}$ Operational images are produced by a machine and are self-reflexive in the sense that they depict both the conditions of observation and what is observed by the machine. In this way, the eigenface image is, in a sense, pure information. Yet, for the human observer, it gives a sense of a modality of machinic recognition, complete with an inherent aesthetic, as is suggested by its description as a "ghost face." In developing the

55 Farocki, War at a Distance. 
concept of the operational image further, specifically with regard to surgical imaging, Aud Sissel Hoel and Frank Lindseth say that they are "generative," that they "differentially intervene," distilling characteristic patterns that would not be seen in other ways. ${ }^{56}$ The eigenface image can also be read in this way, as depicting a process of the statistical pattern recognition of multiple faces through a process of differentiation that results in an operation of recognition.

Sirovich and Kirby claim at one point in their paper that the "eigenpicture" is a way of "making matters more concrete." This claim about the function of the eigenface image relates to its role as a technical image, as Vilém Flusser terms images that structure information in contemporary society and replace other forms of communication. Flusser differentiates the technical image from what he calls "traditional images. ${ }^{n 7}$ Technical images, he says, operate at the "intervals" of understanding. They "translate particles" and "bits of information" that could otherwise not be seen into something that is "graspable, conceivable, tangible. ${ }^{n 8}$ Flusser says that one of the functions of the technical image is specifically to make graspable information that has become abstract through processes of mathematical calculation. Importantly, Flusser describes the technical image as having an ability "to turn from extreme abstraction back into the imaginable. ${ }^{59}$ The concepts of the operational image and the technical image offer ways of understanding the role of the eigenface image within the context of its production, as a part of the operation of recognition. But they also offer a way out: that is, they draw our attention to how the eigenface functions outside of the programming context and within a wider ecology of images that break from traditional notions of representation. In particular, two features of images are useful in

56 Aud Sissel Hoel and Frank Lindseth, "Differential Interventions: Images as Operative Tools," Photomediations: A Reader, ed. Kamila Kuc and Joanna Zylinska (London: Open Humanities Press, 2016), 181.

57 Vilem Flusser, Into the Universe of Technical Images, trans. Nancy Ann Roth (Minneapolis, MN: University of Minnesota Press, 2011), 10.

58 Ibid., 16.

59 Ibid., 21. 
84 Portraits of Automated Facial Recognition

approaching the eigenface method within the context of notions of recognition and identity: their "generative" character and their ability to render things "imaginable." 


\section{Chapter 3: Francis Galton and the Composite Portrait}

The computer existed as a practice before it existed as a machine.

Lewis Mumford, "The Myth of the Machine"

\section{Pictorial Statistics}

One of the things that becomes clear once we shift our approach to the eigenface image from treating it as a computational object of study to an object of study in visual culture is that, as an image, it has a history. Just as Peters traces the merging of statistics with a contemporary understanding of information, here I seek to trace the merging of statistics with vision that has occurred through practices of facial recognition. I do so in order to reveal the specific cultural and socio-political contexts in which this merger came about and developed. This approach runs counter to the assumptions of neutrality and objectivity made on behalf of the technology and instead relates the technical processes of facial recognition to discourses concerning representation. In the 1880 , British anthropologist, statistician and founder of the theory of eugenics Francis Galton began experimenting with what he called the composite portrait. ${ }^{1}$ Galton's practice of composite portraiture can be under-

1 Galton gave numerous talks on his practice of composite portraiture and published articles in Nature. He also described the processes and conclusi- 
stood as an antecedent of the representational mechanism used in the eigenface algorithm. ${ }^{2}$ The process by which Galton constructed his composites was painstaking - a kind of repetition of acts through which the variance of the human hand could fall away. Galton would stack photographic portraits on top of one another and hang them on the wall in front of the camera (Figure 10).

Figure 10: Francis Galton, "Composites assembled for photography" in Popular Science Monthly Volume 13, (August 1878).

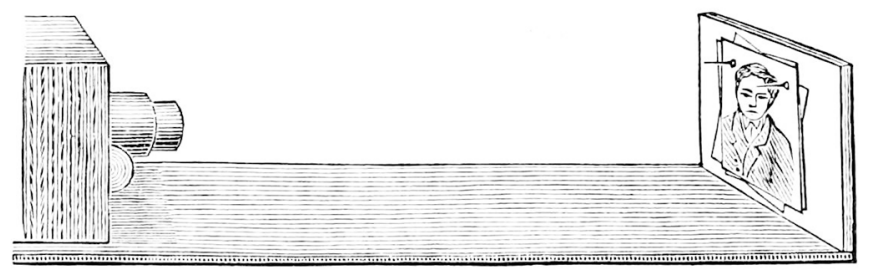

The lighting and scale in each portrait had to be identical, with each face in the same, forward-facing position. Galton made a physical crosshair out of thread, placing it in front of the stack of portraits hanging on the wall. The crosshair ran horizontally through the center of the eyes and vertically through the midline of the face in each image. (Figure 11)

ons of his practice in length in his work Inquiries into Human Faculty and Its Development, 2nd ed. (London: Dent, 1907; galton.org 2018), http://galton. org/books/human-faculty/SecondEdition/text/web/human-faculty4.htm.

2 Interestingly, the inventor of PCA was mathematician and biostatistician Karl Pearson, who was a protégé of Francis Galton and was a central early figure in the study of biometry and social evolution. Yet his invention of PCA, in 1901, was unrelated to his studies of social evolution or to Galton's practice of composite portraiture. See Karl Pearson, "LIII. On Lines and Planes of Closest Fit to Systems of Points in Space," The London, Edinburgh, and Dublin Philosophical Magazine and Journal of Science 2, no. 11 (1901): 55972 . 
Figure 11: Francis Galton, "Step one in assembling a composite photograph" in Popular Science Monthly Volume 13, (August 1878)

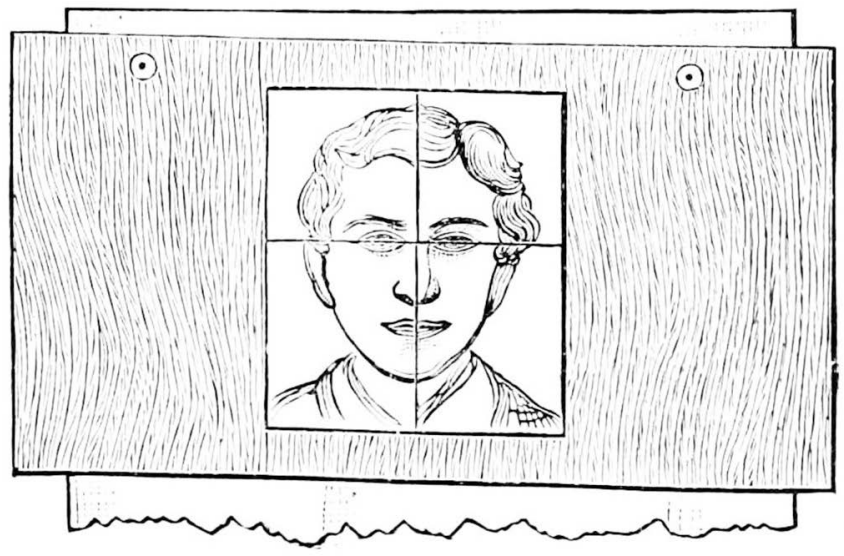

He then took a picture of each facial image using a single photographic plate, closing the aperture of the camera in between each shot, when he would turn to the next image in the stack by hand. The result was that he exposed a single photographic plate to all the photographic portraits hanging on the wall. The exposure time for each photograph was calculated as a fraction of the total exposure time for the photographic plate. For example, if there were ten facial images and the photographic plate had to be exposed for a total of a twenty seconds, each facial image would be exposed for two seconds.

Galton originally applied the composite technique to photographs of landscapes to track changes in topography. He approached images of the human face as unknown landscapes. The late nineteenth century witnessed a seemingly inexorable growth in the urban population. Just as the overwhelming scale of the modern state, according to Peters, raised issues of visibility and knowability, so this population growth created a supposed need to recognize certain segments of this population, such as criminals and other "unknowns," in order for them to "be made visible," and this supposed necessity informed Galton's practice. His composite portraits employed statistics in order to visualize that which would 
otherwise go unseen; they were a statistical table in pictorial form. Galton stated of his composites that "they are rather the equivalents of those large statistical tables whose totals, divided by the number of cases, and entered in the bottom line, are the averages. They are real generalisations, because they include the whole of the material under consideration." ${ }^{33}$ Galton created composites of many sociologically defined groups, including portraits of those who had committed specific crimes, groups of people with particular medical ailments, people from various ethnicities and members of the Jewish population, as well as producing pictures in accordance with idealized categories of beauty and intelligence.

For Galton, the composite's ability to construct representational faces promised a multitude of operational possibilities. Constructing pictures of characteristic physiognomic traits could function as a form of identification and social control. Ultimately, Galton's operation of composite portraits was a failure. ${ }^{4}$ Yet the visual practice of composite portraiture and what it reveals continues to be a source of study and allure today for artists, scholars and scientists. There have been numerous studies and analyses of Galton's composite portraits, which testifies to their ability to continue to evoke curiosity and prompt experimentation in many different fields of study. Galton's use of photography made it possible to freeze a visual process of abstraction. In their book Objectivity, Lorraine Daston and Peter Galison describe the privileged position of the camera in the history of scientific imagery: "machine-regulated image making was a powerful and polyvalent symbol, fundamental to the new scientific goal of objectivity." As they explain, the use of the camera supported a turn, in the 1830s, toward a scientific "devotion towards depicting what was seen on the surface, not what was deduced or interpreted." Additionally, as a visual apparatus, it held a

3 Galton, Inquiries, 233.

4 See Elizabeth Stephens's article on the productive nature of this failure. "Francis Galton's Composite Portraits: The Productive Failure of a Scientific Experiment," unpublished manuscript, June 2013, https://www.research gate.net/publication/323275029_Francis_Galton's_Composite_Portraits_ The_Productive_Failure_of_a_Scientific_Experiment.

5 Daston and Galison, Objectivity, 138. 
"promise of automaticity" in depiction and perception - much like the perceptual algorithms of the present day - which could produce judgment-free reproductions of physical phenomena. Its machinic abilities were thus equated with a level of authenticity.

Galton describes the composite as depicting a thought process. The advantage of the camera, for Galton, lay in its ability to visually represent the abstracting process of statistics in the form of facial images. With the practice of repetitive exposure, that is, in multiplying the reproduction process by photographing the photograph, Galton's composites transformed the use of the imaging apparatus into a form of statistical measurement. Galton's use of the camera to apply the abstractive process of statistics resulted in a depiction that, as Daston and Galison put it, "passed from individual to group." The use of the camera made it possible to repeatedly reference the same, singular portrait in multiple composites. This aspect also allowed for the application of statistical methods to photographic imagery. It also made it possible to construct an image based on an underlying logic of inductive inference, that is, the act of observing many specific cases in order to discern a pattern and deduce a general idea. For Galton, mechanizing the abstractive procedure through the use of the camera provided a perfect way to merge both apparatuses - the statistical and the photographic and thus to make abstract phenomena visible, factual and concrete. The pictorial form of the statistical found in the composite photograph made the generalizations these images contained visible not only to Galton but, as he himself noted, to anyone looking at the composite - a collective vision. This feature made the composite image useful for those guided by the ideals of science. ${ }^{7}$

6 Ibid., 170-71.

7 Galton, Inquiries, 233. 


\section{Zones of Normality}

There are two significant influences that have been mentioned in relation to Galton's approach to the production of composite portraits. ${ }^{8}$ These influences are the theory of abstraction put forward by the philosopher John Locke and a theory of social statistics of which the Belgian sociologist Adolphe Quetelet was the primary proponent. These two influences are pertinent to an analysis of the logic of recognition and its connection to the eigenface method. Firstly, Galton's approach to composites was based on a desire to create representational faces. His interest in representational faces grew out of a philosophical question about the construction of general ideas that originated in Locke's theory of abstraction. Locke examined this question in relation to language and the formation of words, that is, signs that represent general ideas. Locke claimed that general ideas are formed by the mind separating them out from the particulars of context, time and place, which makes it possible for these ideas to represent more than one individual case. In brief, Locke believed that generalizations can be formed by simply leaving out what is particular to each case. Galton took up this line of thinking in relation to his studies of physiognomy, the "science" of judging a person's character, behavior and personality based on their physical features. Physiognomy often focused on the face as the part of the body that could supposedly reveal behavioral characteristics that lay underneath. Galton applied the theory of abstraction to faces, attempting to create a general face as a sign that could stand for a group. Galton developed his own theory, which he coined "eugenics" - he was the first to use the term - based on a belief in a kind of rascist genetic determinism.

Galton's practice of the composite image was situated within a specific socio-historical context. Allan Sekula describes Galton's work with the composite portrait as grounded in the codified theory of "social statistics," which focuses on social behaviors that can only

8 Thanks to James Conant for discussions about his research into the philosophical underpinnings of Galton's composite portraiture practice. 
be observed and identified through statistical means. ${ }^{9}$ A central conceptual category in social statistics is that of "the average man, ${ }^{, 10}$ a notion that comes from Quetelet. Quetelet depicted this concept in the form of a statistical graph, a bell curve in which the average lies at the apex (figure 12). Sekula explains that Quetelet came to regard this pattern as "the mathematical expression of fundamental social law," essentially treating regular occurrence as evidence of a truth. ${ }^{11}$ Most importantly, Quetelet defined the central portion of the curve as a "zone of normality" such that deviations too far from the apex would fall into categories of "biosocial pathology" and "monstrosity."12

Figure 12: Adolphe Quetelet, "Binomial distribution, 999 trials, histogram," in Lettres sur la theorie des probabilites appliquée aux sciences (Bruxelles, M. Hayez, 1846): 103

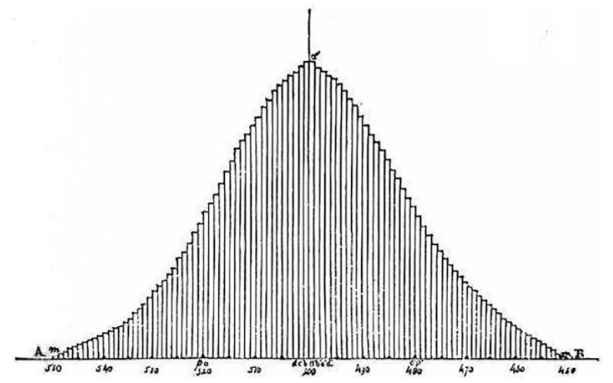

Particular social groups of the population were thereby defined as categories through their deviation from the zone of normality. Here we find an origin, based on statistical logic, for the normative categories that define a process of recognition. Sekula describes the formation of the concept of "the average man" as based in part on

9 Robert A. Nisbet, "Social Science," Encyclopedia Britannica, September 27, 2018, https://www.britannica.com/topic/social-science/The-2oth-century.

10 Allan Sekula, "The Body and the Archive," October 39 (Winter 1986): 19. [364]

11 Ibid. 22.

12 Ibid. Sekula is here quoting Adolphe Quetelet, Lettres sur la théorie des probabilities appliquée aux sciences marales et politiques (Bruxelles: M. Hayez, 1846). 
aesthetic values, borrowing from "art historical evidence of noble Grecian foreheads" and a "racist geometrical fiction."13

Deviations from a norm created another set of sociological categories. Particular medical ailments and criminal behaviors were societal deviations from a norm, and, as part of his eugenics project, Galton wanted to relate typical cases of these to sociological theories of heredity. The eugenics project was taken to its eventual extreme in its ideological and genocidal adoption by the Nazi regime in Germany. As part of an attempt to construct a cultural aesthetic for the regime, the Nazis' infamous art exhibition "Degenerative Art" was held in Munich in 1937, exhibiting pieces of modern art, including works of expressionism and cubism - what Hitler referred to as "the isms." Along with the exhibition, a catalogue was printed. The basis for this catalogue was a book by Paul Schultze-Naumburg titled, "Kunst und Rasse" (Art and Race). ${ }^{14}$ Schultze-Naumburg's book exemplified how the relationship between aestheticized sociological norms and their deviations can be depicted through art. It included photographic portraits of individuals with degenerative medical ailments that left them with deformed faces. These were placed side by side with modernist, painted portraits. Works by Karl Schmidt-Rottluff, Otto Dix and Amedeo Modigliani served as examples that looked akin to photographic portraits of disfigured faces. This stereoscopic comparison between photographs and paintings was utilized to associate modernist art with deviant societal behavior and ill-health in the human population, essentially accusing modernist art itself of being a sociological deviation. The Nazis interpreted the paintings as direct illustrations of a person's physical, surface deformities, rather than expressions of the subjective emotional states of the artists. The coupling of these works expresses an interesting juxtaposition between two types of image production: the expressiveness of the artist and the mechanical precision of photography. Both provided the Nazis with expressions of supposed deviations from a norm or ideal. Quetelet's statistical-

13 Sekula, "Body and the Archive," 22.

14 Schultze-Naumburg, Paul. Kunst und Rasse, Munich, New York: J.F. Lehmanns Verlag, 1928. 
ly based concept of a sociological norm provided the justification for the notion of deviation. In opposition to this "degeneration" in both art and the human race, the Nazis preferred the "great" German artworks, filled with pieces capturing the physical ideals of the "Aryan" race. This provides an extreme example in which the categories set out in social statistics theory constructed a notion of modern identity.

Both the theory of abstraction and social statistics influenced Galton's descriptions and perceptions of the "type" that emerged from the layers of his composite portrait. The significance of the composite portrait, for Galton, lay in its ability to depict what is statistically salient, the "type." Galton described his composites as a "portrait of a type and not of an individual [...] an imaginary figure possessing the average features of any given group." ${ }^{15}$ As Sekula points out, "type" and "typicality" were words used by Quetelet and closely tied to his application of statistics and the promise of sociological truth. The word "typical" expresses the same idea as the word "generic," with the latter presupposing the existence of a genus. Galton described a type as "a collection of individuals who have much in common and among whom medium characteristics are very much more frequent than extreme ones. ${ }^{16}$ Galton gathered faces for the composites based on what he believed to be common physical traits among a group of persons, and these groups, in turn, were based on his own sociologically constructed categories of ethnicity, race, medical or criminal history and social status. The resulting composites were a way for Galton to visually represent the general norm among these groupings such that the particularities of each individual would fall away. Galton described his own perception of the composites as follows: "All that is common remains, all that is individual tends to disappear. ${ }^{117}$ In this way, the type functioned for Galton as the word functioned in the formation of language in Locke's theory of abstraction: the typical face stands in as a general sign for multiple faces of the same group (Figure 13).

\footnotetext{
15 Galton, Inquiries, 222.

16 Ibid., 230.

17 Ibid.
} 
Figure 13: Francis Galton, Composite Portraiture, 1883

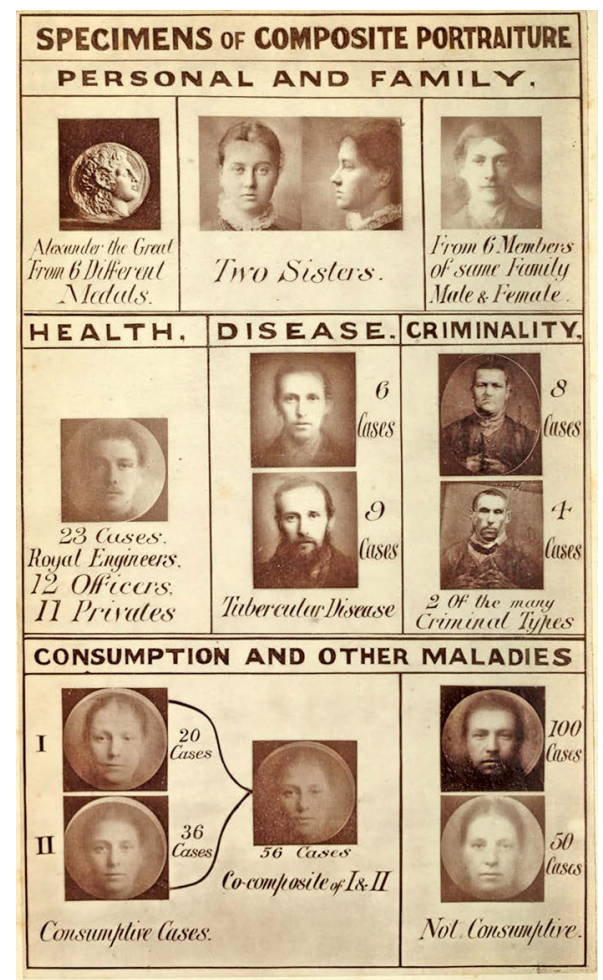

Thus, for Galton, the particular instances faded into the blur of the image:

Those of its outlines are sharpest and darkest that are common to the largest number of the components: the purely individual peculiarities leave little or no visible trace. The latter being necessarily disposed equally on both sides of the average, the outline of the composite is the average of all components. ${ }^{18}$

In this description we can hear Galton adopting much the same approach to interpreting his image as Quetelet did to his graph of the "average man" and the corresponding "zone of normality." Like Gal-

18 Ibid., 223. 
ton's composite, the pinnacle of the bell curve in Quetelet's graph is where clarity and statistical salience lies, with its height depicting the mean. The center of the composite portrait is, for Galton, the significant part of the image. Just as Quetelet had described how, "on both sides of the average," the individual instances decrease and fade, so, for Galton, on all sides of the center of the composite, the particularities fade. At the center of both the graph and the photographic composite you have what is considered by Galton to be a clear impression of commonality, whereas the extremes or deviations from that commonality fade out from the center. The statistically salient "type" is perceived as a visible space in which there is "agreement" and where all the "irregularities disappear, and the common prevails. ${ }^{19}$ The "type" seen at this central mean is able to transcend the blurriness of particulars - all that is rendered ungraspable and unseen by excessive variation - and rise above as a representative face, a face that is able to clearly stand for the characteristics of a given group and, as Galton so succinctly observes, "erase the ghostly blur of difference."

Galton describes the type not as something that is perceived on the surface of the image but rather as something that becomes visible, from beneath the layers of faces, through a process of perceptual emergence. This perceptual emergence was facilitated by certain aesthetic factors with which Galton experimented. For example, he observed that composites are best seen in miniature. In a smaller format, the type may more easily be perceived by an observer. One of the more ambitious aspects of Galton's composite portraiture was his use of the photographic medium - a medium grounded in indexical meaning - for the purpose of producing a representation of a face that does not exist in physical reality. Galton's production of the composite utilized the photographic medium to produce a model. The process of repetitive exposure in Galton's composite portraiture transformed the photographic apparatus into a kind of statistical measuring device. The emergence of a perceivable "type" materialized a distinct figure of the imagination on the basis of which it is possible to recognize individual members of the group.

19 Ibid., 224. 
Thus, this photographic construction of a type is an early antecedent of the virtual models used in AFR technology.

Figure 14: Francis Galton, "Indian Portraits of Alexander the Great with Composite in centre" in Karl Pearson, The Life, Letters and Labours of Francis Galton Vol 2, 1924: 296

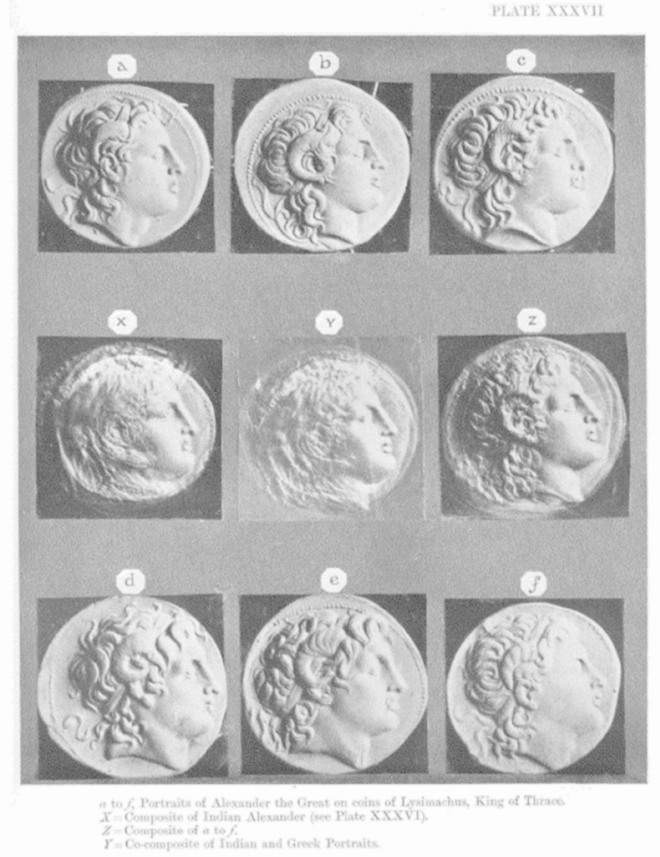

\section{A Sociological Ideal}

Galton's composite image was not only a depiction of a statistical norm. It was also invested with the notion of an ideal form. For Quetelet, the average man was not only a type but also a sociological ideal. Galton's first composite portrait is indicative of this desire for an ideal; it was constructed not from photographs but rather from a collection of medallions featuring the head of Alexander the Great (figure 14). Galton took six separate medallions carved by different 
artists and created a composite from photographic images of each of them. He explained that each medallion contained individual faults caused by the artist's mistakes, but, once composited, all these mistakes fell away, leaving a "pure," idealized representation of Alexander the Great. Galton later applied this same desire for an ideal to produce composites reflecting norms of intelligence and of feminine beauty (the latter made up, exclusively of young white women).

Galton's most well-known composites were of criminals. Criminal portraiture, with its consistent format, provided an ideal source for composite production. A composite Galton made of the "violent criminal" type (figure 15) provided him with an opportunity for deeper reflection on the composite's ability to produce an ideal. He stated:

It will be observed that the features of composites are much better looking than those of the components. The special villainous irregularities in the latter have disappeared and the common humanity that underlies them has prevailed. They represent not the criminal, but the man who is liable to fall into crime. ${ }^{20}$

Galton's goal was to produce a generalization that would depict the features of a violent criminal, but instead he saw an ideal, a face devoid of what he understood to be criminal features. Instead, he took the composite to depict what a man looks like before he commits the violent act. Galton supposed that the irregularities linked with deviation from a norm had faded from the image, taking with them the deviant characteristics of the criminal. The criminal composite projected, rather, a temporal reversal: that is, the face of a criminal before the crime. This was an early recognition of the preemptive and predicative capacity of the composite, statistical form.

20 Ibid. 
Figure 15: Francis Galton, Frontispiece for Havelock Ellis, The Criminal, (New York: Scribner \& Welford, 1890)

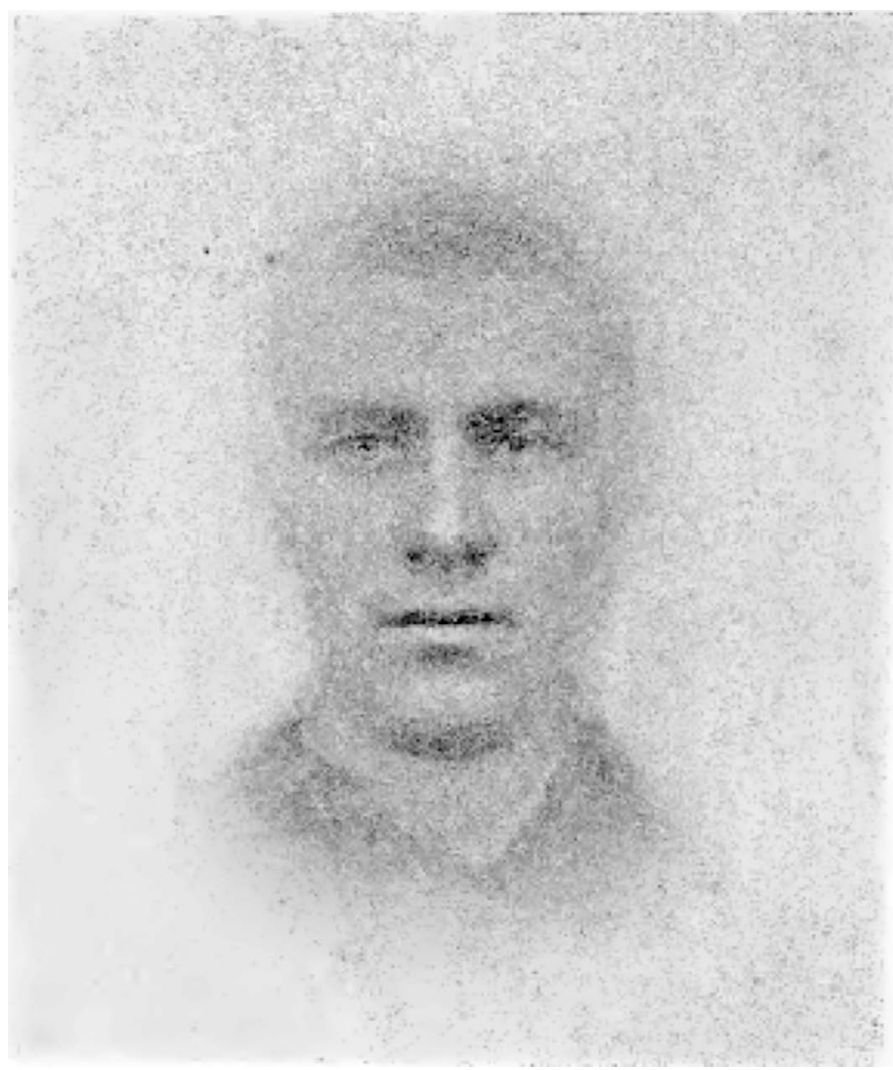

The connection between AFR technology and historical practices of recognition has been noticed by a few contemporary scholars. In this context, mention is often made of the work of Alphonse Bertil$\operatorname{lon}^{21}$ and his system of recognition within criminology. For example, Kelly Gates relates contemporary AFR systems to Bertillon's work on the basis of an underlying "individualizing logic" that has, as its

21 Alphonse Bertillon (1853-1914) was a Parisian police official who developed an anthropometrically based identification system for tracking recidivism in criminals. 
aim, "to identify individual faces rather than facial types." While it is true that the eigenface method, along with all contemporary automated systems of facial recognition, aims to recognize the individual and not the type, I focus rather on the shared logic of recognition present in Galton's composite portraits and the eigenface method, a logic based on a reductive and statistical way of seeing. The analogy between Galton's approach and the eigenface method is brought home when one sees eigenpictures with captions such as "the average man" in journal articles on the method's development. We may also detect an echo of Galton's process of making a composite in Sirovich and Kirby's description of the process of building a training set. Most importantly, the same process of reduction that occurs in Galton's perception of the composite is utilized as a representational mechanism in eigenface.

As I have explained, Galton's composite images made it possible to perceive the characteristics of an individual to pass into the group. Through the recognition process of eigenface and the function of the eigenvector, the characteristics of the individual are extracted from the group. Both occurrences of recognition rely on a composite form; in the eigenface algorithm, this is manifested as the eigenvector, which functions as the primary referent for recognition. Individuals are recognized (or not) based on their deviation from an average, that is, the eigenvector. Although eigenface is not used to produce and recognize a type, this classifying logic lives on as a "ghost in the machine." Built into the representational mechanism of eigenface is the ability to recognize individuals in virtue of the degree to which they deviate from a norm. ${ }^{23}$ In the example of Galton's composite portraits, part of his broader theory of eugenics, we can see the particular cultural and historical context behind the merging of statistics and visual perception we find in the logic of eigenface.

22 Gates, Our Biometric Future, 20.

23 See further discussion on this in Chapter 9: Conclusion, p. 182. 



\section{Chapter 4: Wittgenstein and the Composite Portrait}

Galton's approach to the composite image has mostly been analyzed in relation to social statistics. Yet, as I have mentioned, another significant influence on Galton's practice of composite portrait production was Locke's theory of abstraction. The composite and its process of perceptual emergence is also an object of study within philosophy. The philosopher Ludwig Wittgenstein produced a Galton-inspired composite portrait in 1928. The meaning that he drew from the composite image was quite different from the meaning Galton found in it. Like Galton, Wittgenstein approached the composite image as an image of a thought process. Yet he used the image as a tool in his own investigation of language games and the doctrine of "family resemblance" - an approach closer to Locke's study of the formation of words as signs representing general ideas. In his writings, Wittgenstein argued against the theory of abstraction, claiming that knowledge that derives from a logic of reduction is antithetical to the work of a philosopher. He states:

Our craving for generality has another main source: our preoccupation with the method of science. I mean the method of reducing the explanation of natural phenomena to the smallest possible number of primitive natural laws: and, in mathematics, of unifying the treatment of different topics by using a generalization. This tendency [...] leads the philosopher into complete darkness. ${ }^{1}$

1 Ludwig Wittgenstein, Preliminary Studies for the "Philosophical Investigations": Generally Known as The Blue and Brown Books (Oxford: Basil Blackwell, 1958), 18. 
Wittgenstein's view of the meaning conveyed through the composite image is, I argue, opposed to Galton's, and in this way it runs counter to the logic of recognition through statistical means. I introduce Wittgenstein in this discussion primarily in order to provide an alternative reading of the composite portrait and, connectedly, to allow for a rethinking of notions of recognition and of the relationship between perception and knowledge. I am by no account a Wittgenstein scholar, and I recognize that his work is part of a wider philosophical canon. But here I want to refer solely to his views concerning the composite image and to his implicit critique of reductive methods of knowledge production, for these bear on the counter discourse I wish to direct against the statistical ways of seeing found in facial recognition.

\section{A Destabilization of Vision}

Wittgenstein often refers to ocular metaphors in describing his goal of conceptual and linguistic clarity. In his investigation into what he calls language games, he looks toward simplified models of language in order to understand the workings and process of language acquisition. The study of language games is in part a way of revealing the processes of thought that underlie the use of words, what he also referred to as "operating with signs." Wittgenstein was interested in understanding the use of primitive forms of language, such as the ways in which a child begins to use words, and in looking closely at the everyday use of language. An important motivation for Wittgenstein's investigations was, as he states, "to bring words back from their metaphysical to their everyday use." For Wittgenstein the meaning of words does not lie in abstract ideas but rather is found through their actual use, which is dynamic and mutable. In order to investigate language in the actualities of everyday use, Wittgenstein also thought it important to possess a philosophical ability to "see" the everyday anew. Wittgenstein states: "philosophy is an activity which involves relearning how to look."

2 Ibid., 16. 
Through his writing and the inclusion of ocular metaphors (such as his well-known reference to the "duck-rabbit"), he advocated a destabilization of perception as a trigger for an ability to see anew. This reference to reorienting one's whole visual perspective was profoundly connected, for Wittgenstein, to the opening up of new ways of thinking and knowing. The composite portrait was one such metaphor, and Wittgenstein deployed it in connection to language games, specifically with regard to the issue of the unity of a concept.

Figure 16: The components of the composite photo: Wittgenstein's sisters Gretl, Helene and Hermine, and Ludwig. OLudwig Wittgenstein Trust, Cambridge

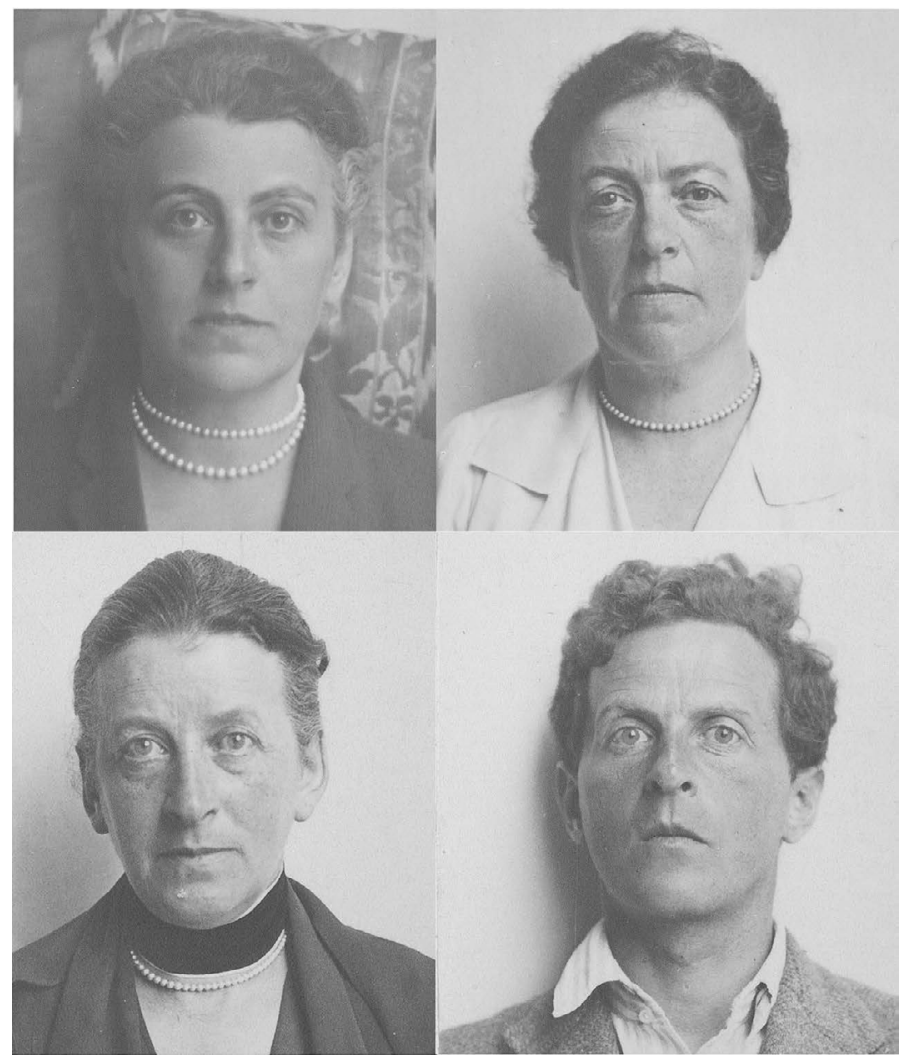


Figure 17: Ludwig Wittgenstein's composite portrait, 1928.

Photographs by Moritz Nähr. (Ludwig Wittgenstein Trust, Cambridge

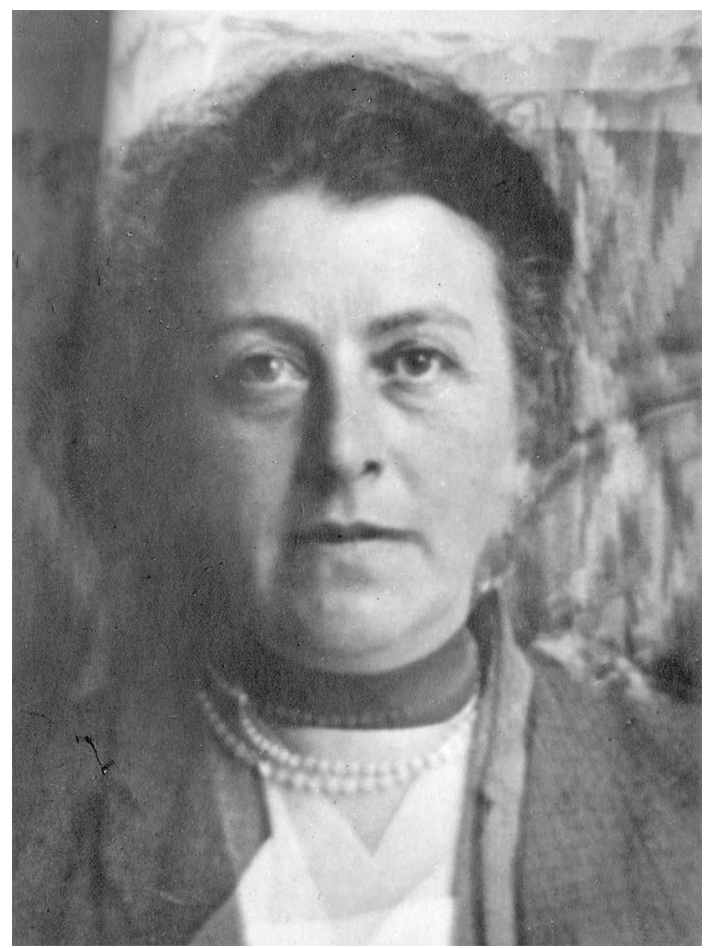

Drawing on Galton's experiments with the composite portrait, Wittgenstein created his own composite portrait with the help of a friend, the photographer Moritz Nähr. Wittgenstein's composite was compiled from photographs of himself and of his three sisters, Gretl, Helene and Hermine (figures 16 and 17). ${ }^{3}$ As one can see in

3 Michael Nedo, of the Wittgenstein Archive in Cambridge, states that the negatives used in the making of the composite no longer exist and the exact frames cannot be located: "Nähr was exposing negatives of four portraits he had taken in a very precise position and with only one background of Wittgenstein and his three sisters onto the same photographic paper. Those negatives no longer exist but from them and/or other negatives which were produced by Nähr in the same context he produced prints some of which Wittgenstein pasted into his photo album, and it is those prints we have got 
the composite image, the four faces are closely aligned, producing an impression of a single person's face in the center of the frame. Looking between the four individual portraits and the composite, one can detect all the features of the individuals within the composite. The composite image and the individual photographs present a perceptual enigma; one detects both the individual features and their integration into a single face. For Wittgenstein the composite functioned as a model of a philosophical method. It illustrates the formation of a concept, which he describes through his doctrine of family resemblance:

Consider for example the proceedings that we call "games." I mean board-games, card games, ball games, Olympic games, and so on. What is common to them all? [...] if you look at them you will not see something that is common to all, but similarities, relationships, and a whole series of them at that. To repeat: don't think, but look! [...] many common features drop out and others appear [...] we see a complicated network of similarities overlapping and criss-crossing: sometimes overall similarities, sometimes similarities of detail. I can think of no better expression to characterize these similarities than "family resemblances"; for the various resemblances between members of a family: build, features, colour of eyes, gait, temperament, etc. etc. overlap and criss-cross in the same way. - And I shall say: "games" form a family. ${ }^{4}$

In this passage, Wittgenstein implores one to look (not to think). In this, he demonstrates an aversion to theoretical and idealized preconceptions, a preference for a pure act of vision over any previous knowledge. After imploring us to look, he then describes a (mental) image of a "network of similarities" overlapping, such as one finds in the resemblances between family members. One could imagine Wittgenstein's composite as such an image. The composite portrait

and they are close enough to the ones Nähr will have used." Email message to author, February 26, 2019.

4 Ludwig Wittgenstein, Philosophical Investigations, trans. G. E. M. Anscombe, P. M. S. Hacker and J. Schulte, 4th ed. (Chichester: Blackwell, 2009): \$66-67. 
depicts the relationships described as a "criss-crossing" of similarities. It visualizes not only the commonalities, and thereby the form of a concept (such as "games"), but also, as Wittgenstein describes, a complex relationship between both similarities and differences, all in an instant.

Wittgenstein mentions the composite explicitly when describing the tendencies in philosophical thinking toward a "craving for generality." He describes as mistaken, the belief that a person who has understood a term - he gives the example of "leaf" - possesses this understanding in virtue of having gone through a visual process of reduction and abstraction from a variety of particular instances, in this case particular leaves. He states:

He was shown different leaves when he learnt the meaning of the word "leaf"; and showing him the particular leaves was only a means to the end of producing "in him" an idea which we imagine to be some kind of general image [...] we are inclined to think that the general idea of a leaf is something like a visual image, but one which only contains what is common to all leaves. (Galtonian composite photograph). ${ }^{6}$

Wittgenstein's reference to the leaf connects his thoughts here to the issue of the particularity of organic forms. Leaves, much like faces, exhibit endless variation. Wittgenstein explains that a general term arises out of an act of looking for a commonality. In describing the formation of a general concept through the example of the leaf, Wittgenstein suggests that generality functions as the end result of language. In this there lies a kind of circular logic and a shortcoming, in that the particularities are all but erased for the purpose of naming, that is, providing a generality. Wittgenstein argues that philosophers who produce such generalities confuse the sign with the object (or objects) to which it points. Wittgenstein describes the job of the philosopher as "purely descriptive." The philosopher,

5 Wittgenstein, Blue and Brown Books, 17.

6 Ibid.

7 Ibid., 18. 
Wittgenstein says, should not reduce phenomena but describe them in all their varying forms. The tendency to adopt scientific methods of reduction - the "craving for generality" or, as Wittgenstein also puts it, the "contemptuous attitude towards the particular case" runs contrary to this conception of the proper role of the philosopher. ${ }^{8}$ In this, he puts forward an argument against forms of knowledge that aim to reduce variation through tools of classification and the statistical promise of finite calculation.

In these criticisms, Wittgenstein produces a kind of intervention - he invites us to engage in a perceptual inquiry into the space between the sign and the objects it names. The sign is loosened from the grip of fixed ideas. In this space of inquiry, there is a fluidity of meaning, possibility and variation. Thus, Wittgenstein's sense of what the composite shows us is very different from Galton's. Given Wittgenstein's interest in paying attention to particulars, he had an altogether different perceptual interest in the composite portrait. Wittgenstein describes the composite portrait as "a picture of probabilities." The composite is an image of multiple perceptual outcomes rather than a singular probability of a type. The composite image has an ability to exhibit all the particular instances at once. Because of this ability to show all aspects of a concept together, Wittgenstein describes the composite as liberating the eye. Instead of focusing on the center of the image as the source of meaning, Wittgenstein focuses outside the center of the frame, and specifically on the blur in the composite image. This attention to the blur inverts the composite's function of defining a "type," thereby reframing the composite's utility. It is equally important that one is able to perceive all the "particular" cases that spin out of the generality, and therefore the generality is not an exclusion of particulars but rather an area in which they overlap. Instead of seeing one face emerge from beneath the layers of the composite image, Wittgenstein observes the composite's ability to depict variation and the spaces in between faces. In this way, Wittgenstein suggests that

8 Ibid.

9 Ibid., 30. 
there is a kind of perceptual movement present in the composite that Galton did not perceive. Galton's was a static observation.

\section{Aspect Perception and Aspect Blindness}

An idea of Wittgenstein's that relates closely to his approach to the composite image is that of aspect perception. His descriptions of the concept are brief and fragmentary, yet the distinctions he makes in the course of its definition suggest a wider importance. He describes aspect perception as an ability to perceive one form changing into another. He gives the example of perceiving a face: "I observe a face, and then suddenly notice its likeness to another. I see that it has not changed; and yet I see it differently. I call this experience 'noticing an aspect."'10 Although he does not directly reference the composite portrait in his discussion of aspect perception, the reference to recognizing one face's likeness to another echoes his remarks on the doctrine on family resemblance. Wittgenstein describes the moment of noticing an aspect as the "lighting up" of an aspect, ${ }^{11}$ as if it is a sudden occurrence or event. Wittgenstein's moment of "lighting up" is to be distinguished both from some combination of looking and thinking and from an act of interpretation. He describes it as "half visual experience, half thought."12 Rather than interpretation, which involves forming a hypothesis and proving it to be either true or false, aspect perception occurs during what he describes as a "state of seeing,"13 suggesting that he is prioritizing the experience of visual sense perception. In this, he means to separate seeing from knowing and from being directed by language.

When describing aspect perception, Wittgenstein references Joseph Jastrow's duck-rabbit illusion. Aspect perception is not about an ability to see the duck and the rabbit but rather "the ex-

\footnotetext{
10 Wittgenstein, Philosophical Investigations, xi, \$113. (Italics in original).

11 Ibid.

12 Ibid., 207e, \$140.

13 Ibid., 223e, \$250.
} 
pression of a change" between the two. ${ }^{14}$ Wittgenstein also gives an example of an illustration of a box that appears in several places in a book, wherein the accompanying captions describe it differently each time, providing for different interpretations of the box. One sees the box differently each time depending on the words that are used to describe it. In contrast, aspect perception is an ability to see something differently, to see both or multiple objects as they are, that functions not through a shift in thought or words but rather through a perceptual ability to see the change and movement from one form to another. He states: "what I perceive in the lighting up of an aspect is not a property of the object, but an internal relation between it and other objects." ${ }^{15}$ Aspect perception requires not only a recognition of different forms and concepts but also the ability to see the movement and relationships between them. Wittgenstein's concept of aspect perception thus suggests a kind of seeing that allows one to see things as other. This concept relates to an ability to perceive particulars in the composite portrait and a set of relationships in a doctrine of family resemblances in that there is a value placed on an interplay between forms. To better comprehend aspect perception and its relevance to my wider analysis, we can refer to its opposite: Wittgenstein's concept of aspect blindness.

Wittgenstein highlights the importance of the will of the observer in being able to see difference aspects. Seeing an aspect is a voluntary act and, as Wittgenstein puts it, it is "subject to the will."16 Moreover, he poses the possibility of someone having "aspect blindness." Wittgenstein describes the "aspect blind" as those who are engaged in a non-dynamic "continuous seeing." It is described as a failure to "be struck," as a perceptual state in which one "keeps on seeing the same."18 Wittgenstein asks: "Could there be human beings lacking the ability to see something as something - and what

\footnotetext{
14 Ibid., 222e, \$130. Thanks to discussions with Tom Mitchell for pointing this out.

15 Ibid., 223e, \$247.

16 Ibid., 224e, \$256.

17 Ibid., 162.

18 Ibid., 56e, \$129.
} 
would that be like? What sort of consequences would it have? [...] Is he supposed to be blind to the similarity between two faces? And so also to their identity or approximate identity?"19 Although Wittgenstein does not write very much about aspect blindness, the primary feature of this (conceptual) condition seems to be an inability to see things as otherwise. His brief descriptions of aspect blindness strike me as an appropriate description of the perceptual emergence of a type in Galton's composite portrait. For Galton, clarity in the composite portrait is made possible precisely through what Wittgenstein describes as a state of "continuous seeing." It is also precisely this ability to "keep seeing the same" that allows for the emergence of a type and, connectedly, that allows for Galton to find a perceivable, clear meaning in the composite portrait. And yet, for Wittgenstein, this is a form of blindness. For him, the source of perceptual clarity in the composite image is to be observed in the blur. It is in the zones of indistinction that the dynamic perception of aspects is possible.

\section{Negotiations of Recognition}

What significance does Wittgenstein's approach to the composite portrait and his concepts of aspect perception and aspect blindness have for the notion of recognition and for the analysis of AFR technology generally? Wittgenstein's work inspires a possible alternative approach to the perceptual meaning of the eigenface image. Wittgenstein's thought runs counter to the reductive logic of statistics that is conveyed by and realized in Galton's composite portraits, and instead he presents an account of the qualities of visual perception and their ability to present alternative pathways of thought and knowledge production. For Wittgenstein, visual perception is a dynamic engagement that allows for a constant renewal in understanding - something that language and words alone cannot do. Taking Wittgenstein's approach as my point of departure, I confront the composite image's contemporary manifestation, the

19 Ibid., 224e, \$257. 
eigenface image. The eigenface image may be seen as a moment of machinic aspect perception, a moment frozen between the multiple probabilities of recognition and the actualization of its operation. The eigenface image, expressive of a statistical pattern, is also an image of probabilities - depicting a point in the algorithmic process of multiple possible outcomes. The blur that constitutes its form is constructed by a collapsed archive of faces, faces that are "known" by the algorithm. At this stage of aggregation in the algorithmic process, the presentation of multiple forms creates, for the human observer, an image on which it is impossible to perform an act of recognition. It is an image that negates singular recognition. This is precisely why it is an image that is expressive of its actual use within the sociopolitical contexts in which it is implemented.

I argue that, as a contemporary production of a composite portrait, the eigenface image conveys a certain truth about contemporary identity. As Btihaj Ajana argues, biometric recognition enacts a form of biopolitics, constructing politicized notions of identity that, in turn, are constituted by a whole set of practices that govern the individual through the hierarchical power relations that not only marginalize the vulnerable, such as the asylum seeker, but also empower those on the opposite end of the geopolitical spectrum, the neoliberal citizenry with a "surplus of rights." ${ }^{\text {"20 }}$ In this highly politicized terrain of mobility, AFR implementation increasingly intervenes to certify identity. And yet notions of identity have never been more uncertain. Individuals who are sorted into highly politicized categories such as "terrorist" can no longer be classified according to fixed social parameters of nationality, ethnicity, racial background or even ideology, but rather are increasingly dispersed among the broader civilian population. As highlighted during the recent migration crisis in the European Union, the abstractions of national sovereignty and national status are undermined when Fortress Europe is confronted with the mobile, fluid identities of a mass and growing population of people defined as "undocumented," a term that signals a loss of all sense of nationhood. The eigenface image, in its composite depiction of multiplicity and blur, corresponds to

20 Ajana, Governing through Biometrics, 5. 
a contemporary socio-political reality: it captures the transience of the subject and the fluidity of identity in mediated form. On this alternative reading, the eigenface image's blurriness is taken to convey a malleability of identity. The faces that are displayed in the eigenface image resist arrest, resist quantification, that is, "concretization," and remain instead dynamic entities. This is not only an alternative, more open way of perceiving these images; in light of the contexts of AFR implementation and the connection between this technology and the construction of contemporary identity, it opens up a space for the exercise of the right to self-determination - a space that is negated by the operations of automated facial recognition.

\section{Concluding Remarks}

In this analysis, I have shifted attention toward the aesthetics of the eigenface image as a way of investigating the notion of recognition that is in play in the operation of an AFR system. In relating the production of these images to composite portraiture, I have sought to problematize the underlying logic of recognition, which is based on a statistical way of seeing. This socio-historical analysis began by tracing this statistical way of seeing and logic of recognition in two images, the eigenface image and Galton's composite portrait. Wittgenstein provides an alternative take on the composite portrait and sees an alternative logic in the image. Wittgenstein's approach reveals how the composite form, which Galton had endowed with statistical relevance, captures a way of seeing that is dynamic, fluid and probabilistic. For Galton, the composite portrait was part of a larger project, his theory of eugenics. For Wittgenstein, the composite portrait was connected to his wider project of a philosophical investigation into language and his doctrine of family resemblance. These approaches contrast with each other on many levels (including in terms of Galton's and Wittgenstein's different fields of study), yet the structure of the composite form is the same in both cases, and it is this same form that structures the eigenface image. Through these different approaches, the composite form has 
emerged as symbolic of the paradoxes that are inherent in a facial recognition process.

Wittgenstein's view of the perceptual intelligibility of the composite portrait and his concept of aspect perception together suggest a re-reading of the eigenface image. On the Wittgensteinian view, it is an image that conveys a resistance to categorization according to the common denominator, a resistance that allows all particulars to remain; the individual does not disappear. Wittgenstein sets out a critique of the logic of reduction that structures statistical recognition, providing alternative conceptual lenses through which to view the operation of machinic vision. The inclusion of his critique in this analysis opens up a space for a perceptual clarity that avoids the algorithm's reductive procedures of statistical recognition. I would like to concur with Wittgenstein in calling for a perceptual intelligibility and a destabilization of vision as I now turn to contemporary art production as it engages with practices of facial recognition - as a source of revelation, recontextualization and reimagining. 



\section{Part II: Artistic Interventions}





\section{Chapter 5: Portraiture in the Age of AFR}

The second part of this study continues to engage in a cultural analysis of AFR technology, but it does so by focusing specifically on the work of contemporary artists. Bringing the overall analysis into the present, this part explores the ways in which contemporary artworks engage with the topic of facial recognition, both revealing and reimagining the cultural dynamics behind its technology and forms of representation. I have chosen three artists, Thomas Ruff, Zach Blas and Trevor Paglen, whose works contribute to a critical discourse on facial recognition and whose work I have chosen specifically on the basis of its engagement with the socio-political contexts and implications of facial recognition practices. Their works shed light on the role of art as a source of theoretical reflection, and they engage experimentally with the technology of facial recognition. Each builds on some of the critical work of contemporary cultural studies scholars mentioned in previous sections, providing nuanced visual articulations of these scholars' contributions.

In the context of the present study, the work of these three artists supplements the foregoing analysis of the eigenface algorithm (with one artist, Paglen, directly referencing this method in his work). Each artist presents an artistic rendering of the composite form, an extended analysis of the role of the composite form and its relationship to facial recognition. These artistic articulations of the composite echo Wittgenstein's sentiments with regard to composite images: they reject a reductive reading of the images and instead open up a space for perceptual fluidity. Although all three artists articulate critical perspectives on facial recognition, their approaches to the topic contrast greatly with one another. They each approach 
the topic through a variety of mediums, a fact that itself reflects the range of meanings attributable to facial recognition and possible responses to this phenomenon. Together they provide a wide spectrum of views on facial recognition and its use in society today.

Growing numbers of artists are working with facial recognition technologies and engaging, more broadly, with the sociopolitical issues surrounding surveillance. Thanks to imagery that allows for a more nuanced and open-ended approach to a topic, artistic engagements with facial recognition technology are able to articulate some of the more complex issues that arise in this area. As the development of AFR systems continues to be, for the most part, guided by the sorts of contexts in which these systems are used, such as policing, the military and marketing, artists engaging with facial recognition technology appropriate the technology and remove it from these original contexts; in doing so, they not only highlight the technology's political and social implications but also imagine alternatives to the use of AFR processes and production.

Art production, being rooted in the relationships of the visual, is in a unique position to articulate the underlying questions of this analysis, which have to do, again, with the forms of visuality on which AFR systems are based and the transformations in cultural meaning associated with their operation. Although the eigenface method produces an image in the course of its operation, most other AFR methods do not. AFR systems and the other forms of machinic vision used in networked surveillance systems often operate in a clandestine fashion and, as such, result in a kind of aesthetic defined by absence, that is, by a lack of images and visual information. For this reason, the artworks that engage with these technologies produce a visibility where there previously was none. Artists such as James Bridle, Omer Fast and Hito Steyerl and the collaborative group Forensic Architecture have addressed these issues in relation to surveillance technologies more broadly. ${ }^{1}$ These artists provide a

1 See James Bridle's "Drone Shadow" series, images of which are available at "Drone," James Bridle website, accessed June 3, 2019, https://jamesbridle. com/works/category:drone; Omer Fast's short film 5,000 Feet is the Best (2011), digital video, http://www.gbagency.fr/en/42/Omer-Fast/\#!/5-০oo- 
kind of "counter imagery"; that is, they produce images that counter a lack thereof. Their work is imbued with a form of political and social agency that is enacted through this visibility. Artists who address this lack of visibility in surveillance technologies use various strategies to do so, including playing with the visual syntaxes of documentary images, merging the physical world with imagined worlds constructed through virtual imagery. These artistic engagements with contemporary surveillance technologies provide a crucial component that is missing from the academic cultural analysis of AFR and surveillance technologies. They provide images that both problematize and reimagine the use of these technologies. In addition, many artistic works contrast with scholarly cultural analyses of the technology in that they leave the implications and outcomes of these issues open ended and in flux.

At the intersection of artistic intervention and AFR technology is an ability to make visible what may be latent in the technology. As well as an operation of recognition, AFR systems involve an operation of representation. The relationship between these two is unsettled. As I explained in the previous chapter on eigenface, the algorithms used in AFR systems require facial models that are constructed on the basis of training data. These virtual models function as a source of face knowledge for an AFR system. These systems rely on representational mechanisms that structure the knowledge acquisition of the algorithm. These representational mechanisms are related to a discourse to do with the representation of faces in the art historical genre of portraiture. Art curators have noticed this relationship. Curator Cornelia Kemp argues that the facial representations found in AFR processes are particularly connected to a cultural history of photographic portraiture. She explains,

Feet-is-the-Best/site_video_listes/88; Hito Steyerl's video How Not to Be Seen: A Fucking Didactic Educational .MOV file (2013), digital video, https:// www.artforum.com/video/hito-steyerl-how-not-to-be-seen-a-fucking-didactic-educational-mov-file-2013-51651; and Forensic Architecture's video Drone Strike in Mir Ali (2013), digital video, https://forensic-architecture. org/investigation/drone-strike-in-mir-ali. 
researchers of facial identification have produced a veritable arsenal of alienated photographs using superimposition, collage, reduction, and abstraction in an effort to divulge the secrets of human face perception. The astonishing findings [...] lead back to the metamorphoses of the portrait in art. ${ }^{2}$

Researchers and developers reference methods of art historical practices of portraiture. In addition, artists reference scientific practices of representing the body. Curator Louise Wolthers describes a strategy, connected to photographic portraiture, adopted by contemporary artists engaging with AFR technology when she states:

The reduction of the unruly, material body to an unambiguous representation is a practice in which analogue photography has played a major role, and in which new digital - and camera-less technologies perpetuate. Works by artists [...] emphasize this by rematerializing or disrupting this form of representation. ${ }^{3}$

Artistic engagements with AFR technology continue a metamorphoses of forms of portraiture practice in the digital age, while also examining how these shifting representations of the face relate to contemporary notions of identity.

\section{Phantom Portraits}

Before I enter into an analysis of specific artistic engagements with AFR, I would first like to discuss more broadly the genre of portraiture and its relationship to notions of representation and recogni-

2 Cornelia Kemp, "The Other Face," The Other Face: Metamorphoses of the Photographic Portrait, ed. Cornelia Kemp and Susanne Witzgall (Munich: Prestel Verlag, 2002), 13. [8-13]

3 Louise Wolthers, "Introduction: Watching Europe and Beyond - Surveillance, Art and Photography in the New Millennium," in Watched! Surveillance, Art and Photography, eds. Louise Wolthers, Dragana Vujanovic, Niclas Östlind, (Köln: Walther König, 2016), 18. 
tion. Portraiture as a genre in art history involves a representative depiction of a person and focuses on the face as the central theme. Historically, the subject was often a person with an important social status, and the depiction captured an expression of the subject's character, societal context and internal emotional life. With the advent of photography and the introduction of mechanical reproduction processes, other genres of portrait, other than the artistic portrait, were born, including the identification portrait produced within the context of institutional bureaucracy and policing. Instead of depicting the character of a subject, the identification portrait focuses on the measurable features of a subject's face. Portraiture in the age of machinic vision has made possible still further categories of the genre. Automated facial recognition creates new ways of reading the face, exploiting perspectives that, because of their scale, are inaccessible to human recognition. These potential new forms of portraiture, that is, new forms of facial representation, can be understood as responses to the technology, to the act of recognition, or as produced by the technology itself.

For example, the artists Adam Broomberg and Oliver Chanarin have termed one such new form of portraiture the "non-collaborative portrait": that is, the production of a facial image of a subject who is "neither consensual nor necessarily aware of the camera." 4 Broomberg and Chanarin explain that this term originated from discussions with engineers who used the phrase to describe the challenges they faced in designing AFR technology. This term brings to the fore issues of privacy, surveillance and consent in relation to the everyday visibility of the face as a potential site of trespass. Broomberg and Chanarin use this term as the title of a series of portraits they produced using a facial recognition system that creates a kind of three-dimensional mapping of faces. In this series of portraits, the subject's gaze is directed off into the distance. They state: 'there is never a moment in the capturing of the 'image' when human contact is registered; the subject's gaze, or any connection between photographer and sitter that we would ordinarily

4 Adam Broomberg and Oliver Chanarin, "The Bone Cannot Lie," in Adam Broomberg and Oliver Chanarin, Spirit is a Bone (London: Mack, 2015), 207. 
rely on in looking at a portrait, is a complete fiction in this space."5 In appropriating this term to provide a conceptual framework for their artistic research, Broomberg and Chanarin underline a central aspect of the visuality of AFR technology as it contrasts with the more stable understanding of the genre of portraiture: that is, the absence of a visual reciprocity. ${ }^{6}$

Algorithmic artifacts such as the eigenface image analyzed in the previous part of this study present yet another new kind of "portraiture" in the age of AFR. Unlike Broomberg and Chanarin's "non-collaborative portrait," the eigenface method is dependent on the forward-facing pose and neutral expression of its subject, and the consent of the subject is obtained. Present in the eigenface image is a history of representations of the face in photographic identification portraiture. What is different, and apparent, in the eigenface portrait is the presence of the perspective of the machine. In 2003, the artist and filmmaker Harun Farocki used the term "phantom images" in an essay of the same title. The phrase had been used, he says, to describe cinematic shots from the 1920s, taken from a perspective "that a human cannot normally occupy." Farocki gives the example of a movie camera attached underneath a train, giving the viewer an ability to experience a vantage point that a human could not physically occupy. The phantom perspective can be described not only as lying outside the limits of human physicality but also as beyond the scale of human visual sense. With Farocki's concept of phantom images in mind, I propose that another category of portraiture has emerged in the age of AFR technology: the "phantom portrait." The phantom portrait is the result of the biometric reading of faces, perceived from the perspective of a disem-

5 Ibid.

6 This lack of visual reciprocity may been seen in earlier artistic photographic portraiture, for example in Helen Levitt's historical subway photography. See Helen Levitt, Manhattan Transit: The Subway Photographs of Helen Levitt (Köln: Walther König, 2017). Yet, Levitt's photographs depicted an emotive character of her subjects. For Broomberg and Chanarin, this lack of visual reciprocity is a defining element within the contexts of facial recognition and surveillance.

7 Farocki, "Phantom Images," 13. 
bodied, machinic eye. Although it is characterized by a non-human perspective, the phantom portrait depicts the human form of the face and presents the visualization of a mathematical abstraction. As part of a reexamination of the genre of portraiture in relation to automated facial recognition, the notion of the phantom portrait raises questions of representation and of the relationship between this technology and notions of identity.

The phantom portrait can be understood as a "counter image," specific in its response to AFR technology, providing visualization of the contexts and implications of its use that would otherwise be non-existent in regards to the output of its actual processes. It makes visible what is latent in AFR technology: ambiguous forms of facial representation that fall within the cracks of its operation, taking into account the "unruly materiality" of the body and disrupting the standardized procedures of facial recognition. Artists such as Bang Geul Han, Heather Dewey-Hagborg and Kristoffer $\emptyset$ rum continue to reformulate the genre of portraiture through the use of varied technological recognition techniques other than AFR, such as facial expression recognition systems, DNA phenotypes and facial tracking systems, respectively. ${ }^{8}$ The artists I will discuss in the following section, Ruff, Blas and Paglen, reference the perspective of the machine that reads faces, a perspective specific to facial recognition practices. One of these artists, Ruff, does not directly reference AFR technology, but his explorations of identification portraiture are pertinent to a wider analysis of notions of recognition and representation. His work also bridges the historical discourse of representation in photography with forms of representation by the technology of AFR systems. These artists produce forms of portraiture that disrupt traditional representations of the face and, as a result, articulate various connected issues concerning the politics of representation that are inherent to the contexts in which facial recognition systems are applied.

8 See Bang Geul Han, Referential Gaze (2015), inkjet prints; Heather Dewey-Hagborg, Stranger Visions (2012-2013), sculpture; and Kristoffer Ørum, Ambivalent Physiognomy (University of Copenhagen, May 22, 2017), performance. 



\section{Chapter 6: Metaportraits: Thomas Ruff, andere Portraits}

\section{Background: The Composite Portrait in Contemporary Art}

I have already discussed the composite portrait as an object of study within the fields of science and philosophy. Yet the composite portrait has also been an object of study within the field of art. Instead of using it to construct sociological or criminological taxonomies, artists have usually approached the composite portrait as a visual construct, problematizing the relationship between mechanical or digital reproduction and indexicality. One of the most well-known artists to work with composite portraits is Nancy Burson, who, in the 1980s, produced a series of works that featured the composite image and exploited its ability to produce ideals and types. Burson curated the categories of her composite portraits, constructing digitized averages of faces that portrayed sociological ideals of gender, power and race. These works expressed the critical role of identity politics in defining the sociological ideals. For example, Burson constructed a composite portrait, Beauty (1982), which consisted of the faces of famous Hollywood actresses, resulting in a depiction of a feminine and racialized ideal of beauty. Another work, Warhead (1982), is a composite of the faces of the global leaders who were, at the time, antagonists in a Cold War standoff, depicting an ideal of a political power defined by the white male. One reading of Burson's composites is that they invert the notion of the ideal that Galton had pursued through his composites, instead revealing the racialized and gendered parameters within which concepts such as beauty 
and power are imagined in our society. In this way, Burson is able to use the symbolic aspect of the composite in a critique of society.

Burson's composite portraits are considered some of the earliest computer-generated artworks, and aesthetically they exhibit a digital seamlessness in which the visualized average can be observed in a singular yet pixelated face. Thomas Ruff produced a less well-known series of composite portraits in the mid-1990s, the andere Portraits, utilizing analogue photography and silk-screening processes to highlight the disparities between the composited faces. Rather than focusing on the composite's ability to produce and depict a monolithic aggregate, Ruff's composites call our attention to a constant movement between faces and thus make the zones of indistinction obvious.

\section{Identification Portraiture}

Ruff's andere Portraits grew out of his earlier series Portrait, an investigation of identification portraiture. In the late 1980s, Ruff began producing portraits of his colleagues at art school in the style of ID card and passport photos. These images grew into a study of identification portraiture, the kinds of photographs that function to identify individuals based on a clear, forward-facing pose and a neutral facial expression that accentuates the measurable features of their faces. These portraits explicitly exhibited photography's ability to show only the surface appearance of the subject; rather than revealing something about the person, as the viewer would expect from an artistic portrait, they left out the context, character and personality of the individual. These portraits exaggerated this capacity of the photograph to depict only what lies on the surface. They discouraged any internal reading of the photograph's subjects.

I argue that Ruff and Wittgenstein share a similar approach to the investigation of their respective mediums, photography and language, both of which are ways of operating with signs. As I mentioned previously, Wittgenstein said that in his investigation he was aiming to "bring words back from their metaphysical to their everyday use," that is, to understand language through a study of its 
actual and everyday use. I argue that this approach is similar to how Ruff approaches photography and the photographic image. His investigations with photographic images attend very much to their everyday and bureaucratic uses, extracting the photographic image from a discourse on indexicality and instead approaching photographs through their use and as a kind of visual construction. Ruff states that photography "can only reproduce the surface of things." Far from understanding this as a limitation of photography, Ruff uses his work to highlight this aspect of the medium. This, for me, also harks back to Wittgenstein's approach to language; he cautions against the view that, as he puts it, "the incomparable essence of language [...] was hidden from us beneath the surface." Instead, Wittgenstein proposes to liberate us from this urge by getting us to recognize that everything "already lies open to view."

Ruff's work with identification portraits conveys a sense of the chasm between an observer of the image identifying with the subject's face and identification as a practice of governance and institutional regulation. Ruff's photographic work is an investigation of the utilitarian uses of photography, or, you could say, of how photography is used in the everyday. His identification portraits concern types of photographs that, in some way, otherwise go unnoticed; they are both ubiquitous and unquestioned, utilized for specific and usually bureaucratic purposes. What is on display in Ruff's portraits is not only the subject's face but the conditions of its representation. In this, Ruff takes the visual language of the archive to represent the individual. Placed in a purely visual realm of art, Ruff's series of identification portraits problematizes the authority the archive has in relation to the subject of the portrait. As Dan Adler puts it:

1 Richard Dorment, "Photography in Focus," The Telegraph, May 29, 2003, http://www.telegraph.co.uk/culture/3595514/PHOTOGRAPHY-IN-FOCUSThe-deadpan-images-created-by-Thomas-Ruff-of-nameless-individualsand-equally-anonymous-places-are-masterpieces-of-austere-neutrality. -By-Richard-Dorment-Now-for-something-completely-indifferent.html and Hilde Van Gelder and Helen W. Westgeest, Photography Theory in Historical Perspective (Hoboken: Wiley-Blackwell, 2011), 43-44.

2 Wittgenstein, Philosophical Investigations, 126, \$92. 
Everything and everyone that enters the archive is said to be subject to the same ritualized process of framing, a process that creates (and maintains) order, that suggests systematicity but refrains from signification. [...] Ruff [...] rigorously and repetitiously enacts photographic technologies, with each body of work implying context of regulatory ordering and categorization, an operation that must operate by both including and excluding subjects. ${ }^{3}$

One of the results of Ruff's reenactment of the archival ritual of identification photography is the conversion of his photographic subjects into objects, as Adler's reference to refraining from signification suggests. Ruff has stated that, once photographed, the person is "thingified." The exclusion of a subjective stance in the image occurs through the observation of the image; the photographed subject lacks the character that is usually expected of a portrait in an artistic context. Instead, Ruff's portraits evoke the role of photography in science, as a way of documenting truth, and when applied to the human subject the result is a tension between a truth of the subject and the truth of the image.

Ruff's Portrait series visualizes what the surveillance scholar Lyon describes using his concept of a "data double," which is a person's identity within a database, an identity created from coded categories that produce a kind of "virtual fiction." These identities circulate amongst multiple institutions and interests and, all the while, are "vulnerable to alteration, additions, merging and loss as they travel." The production of a "data double" results in a gap between a sense of self and a "data image." Ruff has described his portraits as a kind of "fiction," and his portrait work has been criticized as producing an experience of alienation rather than identification in the viewer. Yet this experience is what we might expect of a subject confronting their own "data double" - an experience of dissonance

3 Dan Adler, "The Apparatus: On the Photography of Thomas Ruff," Art Journal 75, no. 2 (Summer 2016): 68. [66-87]

4 Thomas Ruff, Andere Porträts +3D (Germany: Cantz Verlag, 1995), 16.

5 Lyon, "Surveillance as Social Sorting," 22.

6 Ibid., 27. 
produced by the representation of themselves as information. Lyon describes the "data image" as a digital persona that is created on the basis of risk assessment and then circulated and as an example of how surveillance communication "is increasingly distanced from the person from whom the data is initially obtained." Lyon explains: "The data-image may be abstract but it is not innocent." In the way that they bring to the surface the glaring bifurcation of the observer's sense of identity through the photographed subject, Ruff's Portraits may be understood as illuminating the aspects of data images described by Lyon. Ruff's study of identification portraits reveals that these forms of representation are not neutral in much the same way that, as Lyon explains, data images are not innocent.

\section{andere Portraits}

The composites in Ruff's andere Portraits (1994-1995) were constructed from his earlier portraiture series. These portraits extend the idea of a photographic "fiction" underlying the image. He has explained that, with these portraits, he intended to construct faces:

The original idea was to reconstruct one of my portraits from several faces. But I gave up the idea quite soon, it was impossible. I developed the idea of "building" new faces, faces that do not exist. I mixed - man and man - man and woman - woman with woman and woman with men. They should be believable but at the same time the viewer should realize they are fictional. ${ }^{8}$

Ruff produced these composites by appropriating a security camera apparatus called the Minolta Montage Unit, used by the State Bureaus of Investigation in Berlin in the 1970s. This camera was originally used to produce pictures that combined individual facial features from four photographs, based on an eyewitness account of

7 Lyon, "Facing the Future," 173.

8 Thomas Ruff, email message to author, May 15, 2016. 
a crime. The decision about which faces to include in the composite was made on the basis of the eyewitness account. Instead of having an artist sketch the face, the unit could composite different facial features to create a photographic "sketch" of a criminal's face. The resulting composites were often used as mugshots and displayed on "Wanted" posters in public spaces.

The Minolta Montage Unit used four passport-sized photos and, by way of an optical mirror system, merged them into a composite. There is a built-in polarizing filter to eliminate the hard edges where the lines of the facial features do not match up. The Minolta unit is a more sophisticated version of Galton's composite practice in the sense that the unit itself mechanizes the process of layering the faces. Instead of using four photos, Ruff combined two faces taken from his Portraits series. In producing these binary composites, Ruff played and experimented with the gender binary, combining faces of men and women. He then took a photograph of the resulting composite and used the photographic slide as the basis for a black-and-white silkscreen. He explains: "I wanted to go closer to the police technique, that's why I decided to print them as silk screens with the technical structure of a printed medium." In contrast to the photographs from which they are constructed, which were originally exhibited by Ruff in vivid, saturated color, the resulting images are black and white. For the andere Portraits, Ruff consciously chose to avoid color photography, noting that a color photograph is much closer to a depiction of how reality looks to the unaided eye.

In his descriptions of this technique, Ruff often refers to the security and policing context in which the Minolta Montage Unit was originally used. His approach to investigating photography and photographic practices often plays with the notion of the photograph as a fact and/or fiction, questioning and problematizing its evidentiary quality. The use of the Minolta Montage Unit to produce mugshots from eyewitness accounts of crimes seems also to merge these notions, revealing the resulting composites to be fictional constructs that make use of the medium of photography. The "other"

9 Ibid. 
referred to in the title of the series - andere meaning "other" in German - can refer to the original use of the apparatus by security bureaus: Other, with a capital "O," denoting those who stand outside of society, the criminals or the terrorists, those outside of the realm of the familiar and deemed dangerous. It can also refer to the face that is created when the two portraits are used to make the composite. As with Galton's composites, the fact that this face has no indexical referent and exists only in a pictorial reality is highlighted. Where the portraits combine the faces of men and women, the "other" can also refer to that crossing of a culturally constructed gender binary, a crossing that creates the face of an "other" gender that fluidly moves between the two traditional gender categories.

\section{Metaportraits and the Binary Face}

Referring to the reproduction technique used in Ruff's Portraits series, that is, the act of producing portraits of portraits, art historian Patricia Drück describes these pieces as "meta portraits."10 This term can equally be applied to the andere Portraits, in that they are portraits of an already existing form of portraiture, the composite mugshot, and they appropriate the Minolta Montage Unit apparatus in their production. I think Drück's term is helpful in understanding these images and Ruff's motivations, because it captures the way in which Ruff is producing portraits of a portraiture practice in such a way as to reveal the practice's character and essence. In doing this, Ruff presents the resulting composite portrait as an object of visual contemplation.

Ruff's decision to use only two identification portraits leads to a particular sort of binary composite. As Ruff states in the passage quoted above, he experiments with gender, combining the faces of both men and women. In this way, one might say that Ruff actually utilizes some notion of a "type," in gendered norms, and creates

10 Patricia Drück, Das Bild des Menschen in der Fotografie: Die Porträts von Thomas Ruff (Dietrich Reimer, 2004), 170, quoted in Gelder and Wehtgeest, Photography Theory, 43. 
composites of faces that disrupt these binary gender norms. In the piece titled anderes Portrait, Nr. 71/65 (1994/95) (figure 18), an incongruity between gendered norms is made especially visible. At the center of the image there is a single face. This face has delicate, feminine features, with rouged lips. Panning out from the center, two separate and incongruent hairlines begin to form. One face appears thinner, with a short, masculine hairstyle. The other face, behind it, creates a kind of halo effect, encircling the other. A wild mane of hair surrounds both faces. From the neck down, there is a single black turtleneck.

Figure 18: Thomas Ruff, anderes Portrait, Nr. 71/65 (1994/95). Image courtesy of the artist

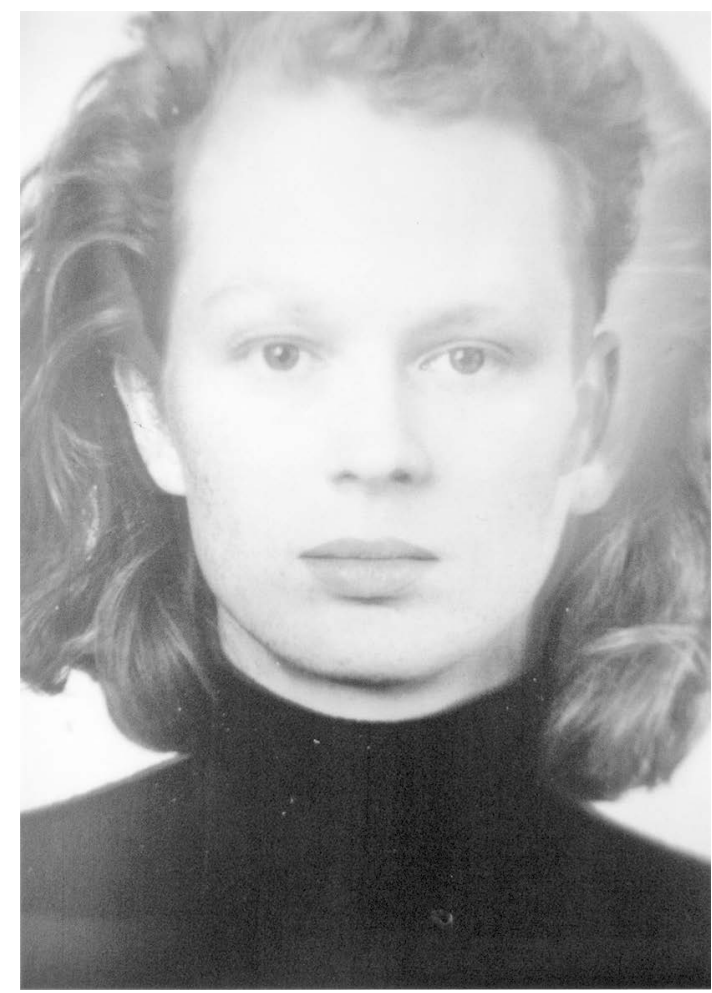


Another piece, anderes Portrait, Nr. 102/13 (1994/95) (figure 19), produces an experience of constant double vision as one observes the appearance of the face. Although, like the previous image, there is a kind of alignment of faces at the center of the image, the features are less sharp, making the face appear to be in constant movement. The outlines of the two faces are closer to each other than in the previous image, and yet they do not align. The result of this lends the portrait a stereoscopic effect, giving the eye of the observer no outline to rest on as that of a singular face. The face at the center of the image appears feminine because of the obvious makeup, mainly eyeliner, eyeshadow and lipstick, as well as cosmetically shaped eyebrows. Yet the overall form and shape of the composite face produces an extremely masculine effect; it is stocky and muscular. The hairline is starkly defined by a dark hair color and parted to the side. As in all the andere Portraits, the facial appearances meld into a singular image below the face. In this portrait, the subject has a broad neck with a very visible Adam's apple, and shoulders clothed in a man's button-down shirt. This and the previous image are examples of how Ruff's composites highlight the incongruities between the two portraits that make up his composites. Although a single face appears at the center of the images, the misaligned outlines of the underlying faces immediately unsettles the perception of any singular face. A catalogue that was produced for an exhibition of the andere Portraits at the Venice Biennale in 1995 reveals that Ruff produced a few of these composites in stereo, using the same face to produce two different composites. ${ }^{11}$ In the catalogue, two separate composites using one of the same faces sit side by side on opposing pages. This adds another level of stereoscopic vision for the observer; one can see the same face, but differently. The portrait I just mentioned, anderes Portrait, Nr. 102/13 (1994/95), has a kind of doppelgänger in the piece titled anderes Portrait, Nr. 102/125 (1994/95) (figure 20), which uses one of the faces used in the former portrait but combines it with a different face. When placed side by side, one is able to observe the similarities between these two portraits, most significantly in the eyes. But the image gives way, once one turns

11 Thomas Ruff, Andere Porträts +3D. (Germany: Cantz Verlag, 1995), 34, 35. 
to the rest of the face, to the merging and disparity produced by the imposition of the "other face." The shapes of the heads are different, and the clothing worn, which can be seen clearly from the neck down, differs as well. In looking at these portraits side by side, some of the characteristics of the shared face emerge through the comparison, while, at the same time, the binary structure of these portraits is accentuated.

Figure 19: Thomas Ruff, anderes Portrait, Nr. 102/13, 1994-95. Images courtesy of the artist

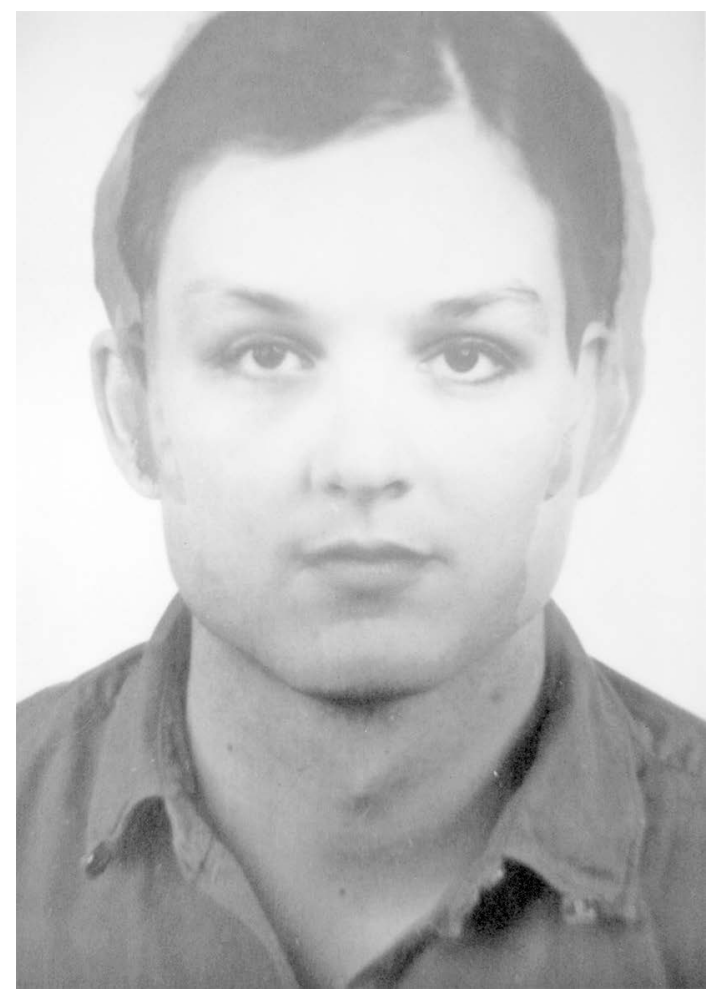

Ruff's andere Portraits consist of overtly and visibly manipulated images. Aside from the many layers that are produced by the reproduction practice used to create the andere Portraits, there are also many layers within the content of the images. Ruff's intervention 
in producing these images is made visible through the use of analogue mediums, both the photographic apparatus of the Minolta Montage Unit and the process of silk-screening. This intervention is made more visible to the viewer in the choice of a binary image structure, because the viewer sees more clearly the outlines of each individual face. There is a seemingly constant movement between faces, a binary face that never stays put.

Figure 20: Thomas Ruff, anderes Portrait, Nr. 102/125, 1994-95. Images courtesy of the artist

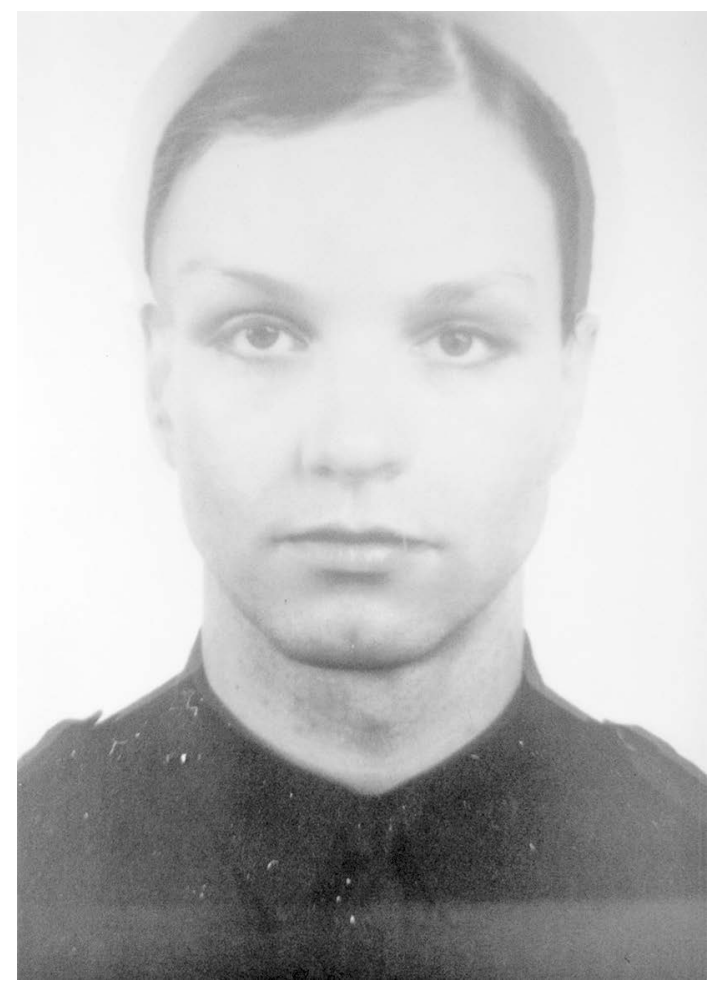

Rather than applying a statistical technique that would merge the faces into a conglomerate, such that the multiplicity of outlines created a blur, Ruff chooses to use just two images and thus makes the misalignment all the more visible. The spaces in between the two 
opposing or incongruent faces become spaces of uncertainty. In the central areas where the two faces align, a third face emerges. This face, a "fiction" as Ruff calls it, is apparent and seen in the photograph, and yet it exists only in the pictorial realm. ${ }^{12}$ Historically, in Galton's use of the composite portrait, this "fiction" was endowed with a level of certainty and statistical salience. Yet, in Ruff's portraits, this "fictional" or "other" face refuses to stand still. In place of certainty, one sees double and then, again, multiple faces. The areas of commonality are unhinged, uncoupled from their surroundings, and produce instead an uncertainty. Once you see a face, it changes and you see another, and then it changes again. Ruff's portraits produce a constant mutability and expose a process of perceptual construction at work in the observer. In this way, the experience of the observer of the image is a part of what is on display in the image.

The technical attributes of the Minolta Montage Unit are described in a trade magazine: "Interestingly, the synthesizer is not hindered by racial or sex barriers. It can be adjusted for skin tone and texture, allowing any feature mix of male or female."13 This is framed as a kind of technological advantage: that the apparatus is neutral and unhindered by social norms. Ruff utilizes this feature to produce portraits that mutate fixed, binary notions of gender. Ruff's constructions of what he calls "fictional faces" present faces that reveal not only the negotiations of a technically produced representation but also the relationship of the representations to the cultural contexts in which identity is negotiated. The andere Portraits display the malleability and ambivalence of identity. Dan Adler points out that the andere Portraits "demonstrate how the misuse of archival technologies may be read as gestures of a progressive political position, one that is open to the concept of exposing (or at least acknowledging) subjects that cannot be (or resist being) classified."14 Ruff's andere Portraits reference the relationships between surveillance by security forces, archival documentation and

12 Thomas Ruff, email message to author, May 15, 2016.

13 Clemens Mitscher, "1987 - Opfer," Clemens Mitscher website, accessed May 25, 2017, http://mitscher.de/content/?page_id=265.

14 Adler, "The Apparatus," 7. 
identity. The use of the binaries that visually structure Ruff's composites may also be understood as a reference to the binaries that problematize the visual operations of recognition, that is, the inner versus the outer, the objective versus the subjective and, as Ruff says, "A truth versus fiction." ${ }^{15}$

\section{Scale}

One of the aspects that characterizes Ruff's work with portraiture is the monumental scale on which he has exhibited some of his portraits. Combining the photographic portrait with the genre of large-scale, life-size painting, Ruff emphasizes the iconic status of the photograph and, as Enwezor argues, "emancipates the photograph from being read as a document, moving it instead towards being perceived optically and approached haptically as a picture."16 The large-scale format for the exhibition of prints was also used by Ruff in his exhibition of the andere Portraits at the Venice Biennale in 1995. This again affected the way in which these images conveyed the meaning of a certain photographic genre of portraiture, in this case, the mugshot, by removing them from the contexts of identification and policing, and instead allowing observers to approach them as pictorial objects of study.

Although they were not exhibited on a monumental scale, the andere Portraits were printed life size so that a viewer, upon confronting them up close, could witness a breakdown in their technical structure through the enlargement of the silk-screened, photographic grain (figure 21). Distance is accentuated through scale. The size of the portraits makes it so that the viewer has to stand

15 Ruff, Andere Porträts +3D, 16.

16 Okwui Enwezor, "The Conditions of Spectrality and Spectatorship in Thomas Ruff's Photographs," in Thomas Ruff: Works 1979-2011 (München: Schirmer Mosel, 2012), 11. [9-19] The monumental scale on which his series of identification portraits was exhibited in Düsseldorf, Germany drew criticism at the time, as it conjured up a cultural memory of a Nazi aesthetic. Thanks to artist Johan Röing, who attended the Düsseldorf school with Thomas Ruff and pointed this out. 
at a distance to properly perceive the entire image - to see it as a complete portrait. The closer one gets to the image, the more abstract the image becomes, further alienating the observer from any identification with the face. The further away one gets, the more the movement between the binary faces is made visible. Rather than depicting a clear image of the constructed face of a criminal, as in the original composited mugshots, it only showcases the construct. The malleability of identity and identification is made evident.

Figure 21: Thomas Ruff, Venice Biennale, 1995. Images courtesy of the artist

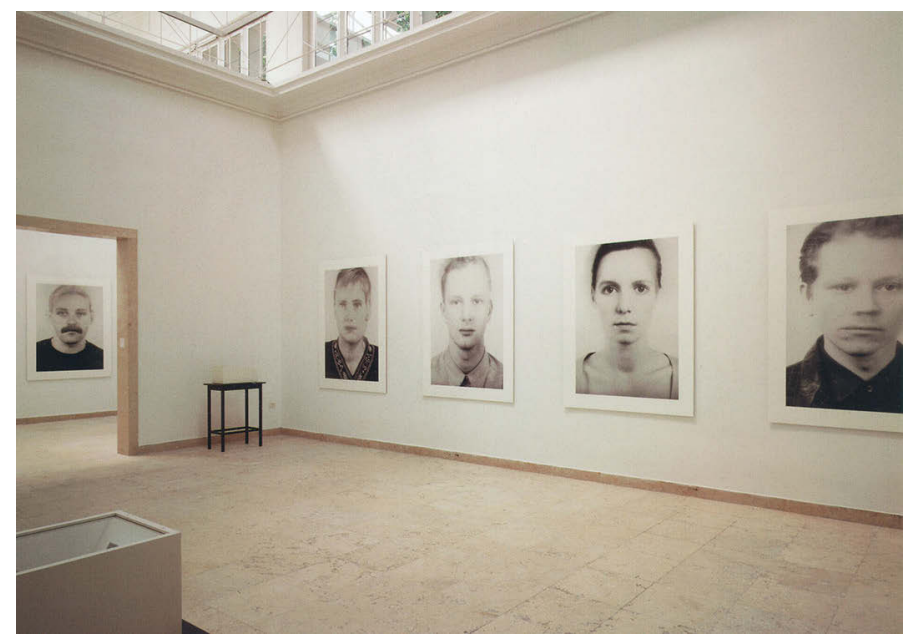

The scale of Ruff's andere Portraits also runs counter to Galton's beliefs about the ideal way of perceiving composite portraits. Galton's research mentions size as an important determinant of the readability of composite portraits, saying that, the smaller the image, the more likely it is that an observer will be able to see a single average face emerge from the composite. Against this prescription, Ruff's portraits instead actively make visible a constant movement, and seem to revel in the inability of the viewer confronting the portrait to nail down anything "identifiable." On this scale, the artificial nature of the photographic image of a human subject gets exaggerated, further emphasizing the limits of the photograph - that it 
can only express the surface of things. Ruff's large-scale exhibition of the andere Portraits reveals everything on the surface, making visible the many layers and details of the images' photographic structures. And yet these images also evade any of the truth claims that are associated with the practice of mugshot portraiture from which he borrows. His work seems to state: just because something is seen, it does not mean it is true. In fact, his work goes further: the more you see, the less the truth can be revealed.

\section{Concluding Remarks}

Ruff's andere Portraits are a perceptual study of the photographic construction of the face. The zones of incongruity between the faces are made visible, provoking a perceptual movement. This both reveals an internal relation between faces and produces a new, "fictional" face. Ruff recontextualizes the use of the portrait as a document, bringing the composite portrait into an art practice as an object of study and positioning it as a site of pure visuality. Yet it is still a portrait, and so the observer cannot help but connect the representation of the face with an identity. In this way, both the face and identity are revealed to be constructs. The binary presentation of faces provokes a constant perceptual movement - the face refuses to stand still. The andere Portraits thus reveal a nuanced understanding of the transience of identity, gender and form. In this way, Ruff's artistic use of the composite is a distillation of the practice of representational resistance to reductive forms of recognition I discussed in the previous section.

Through its appropriation of a security apparatus used for facial recognition, Ruff's artistic practice serves as a precursor to the artworks that I will go on to discuss. The andere Portraits experiment with the truth claims of the photographic document and visualize a dissonance between the subjective and objective processes of identity construction through photographic representation. As an artwork, the portraits call our attention to the processes of perception both behind the image's production and in the experience of the observer. Taken out of an original context of documentation, mug- 
shots and security apparatuses, the andere Portraits point us toward the perceptual processes that are revealed upon the observation of the image, rather than depicting an image for a proposed function or to achieve a fixed outcome. Ruff's portraits provide questions rather than answers. They question the dominance of the visual technologies used in the service of operations of recognition directed toward subjects. In particular, his composites constitute an artistic resistance to the visual act of classification. This theme of resistance to classification is taken up in the work of the following two artists, who in turn extend this line of questioning and critique towards the algorithmic forms of perception and recognition. 


\section{Chapter 7: Faces in Excess: Zach Blas, Facial Weaponization Suite}

\section{Background: A Conceptual Framework}

Discussing his artwork Facial Weaponization Suite (2014), Zach Blas states: "I saw a coterminous rise of masked protest alongside the rise and boom of biometric industries." This connection between obscuring and scrutinizing the face in public life highlights the fact that the face is a site of confrontation between opposing political strategies. On the one side, institutional biometric technologies are employed as a means of regulating individuals through ascertaining their identities. On the other side, activist groups precisely resist identification and regulation as part of a political strategy. Through his art, Blas foregrounds a study of contemporary biometric facial recognition practices as rooted in the socio-political contexts in which they intervene. The series of masks produced as part of $\mathrm{Fa}$ cial Weaponization Suite utilizes both a sculptural and a performative medium, through a series of workshops and events. Blas' critique of biometric recognition involves understanding its technology as enacting a computational process of standardization and as an extended arm of neoliberal political strategies that unequally impact already vulnerable and marginalized groups in society. ${ }^{2}$ Blas's

1 Ben Valentine, "Weaponizing Our Faces: An Interview with Zach Blas," Vice, July 10, 2014, https://www.vice.com/en_us/article/vdpzaa/weaponizing-our-faces-an-interview-with-zach-blas-715.

2 Talk given by Zach Blas as part of the symposium Invisible/Visible, held at the New Museum, New York, March 7, 2015. 
artwork speaks directly to and confronts these aspects of biometric facial recognition, utilizing masks and performance in reference to a history of carnivalesque forms of hierarchical inversion.

Blas's critique of biometric technology is similar to that of scholar Shoshana Magnet, in particular to her call for a broader approach to technological failure. ${ }^{3}$ As Blas states:

Biometric technologies rely heavily on stable and normative conceptions of identity, and thus, structural failures are encoded in biometrics that discriminate against race, class, gender, sex, and disability. [...] Biometric failure exposes the inequalities that emerge when normative categories are forced upon populations. ${ }^{4}$

Blas's work is informed by the theoretical frameworks of queer theory and post-colonial theory, utilized as critical tools through which to approach technology. What Blas argues is that biometric recognition involves a computational process of standardization with set parameters produced through the biases inherent in a history of social discrimination. This computational process, Blas argues, has deeply embedded within it a visuality drawn from societal norms of gender, race and sexuality.

Blas's work speaks to a shift in political strategy in relation to activism and civil disobedience. In the 1970s in the US, the tools of visibility and political representation were the primary vehicles of political agency for people of color, gay activists and feminist groups. Visibility and political representation were, in this context, equated with political agency and legitimization, providing individuals from marginalized communities with a platform to communicate and fight for specific political goals. Along with certain other artists, Blas argues that, with the increasing pervasiveness of contemporary surveillance technologies such as biometrics, visibility and representation have instead become a tools of regulation

3 Magnet, When Biometrics Fail, 9.

4 Zach Blas, Facial Weaponization Communiqué: Fag Face (2012), video, https:// vimeo.com/57882032. 
and control wielded by the state. ${ }^{5}$ These technologies enact a form of societal control through the increasing accumulation of data produced from surveilled bodies. Blas states:

Such a digital regime profoundly inverts the political promise of visibility and representation as means toward democracy and equality. Any exposure of bodies is now usurped as a potential pathway to control and governance, and thus, undoes documentation as a purely liberatory project. ${ }^{6}$

The contemporary techno-political landscape of pervasive surveillance technologies and data accumulation inverts the political agency of visibility. This inversion, in turn, produces an alternative form of contemporary political agency that is rooted in the claim to the right not to be visible, the right not to be recognizable or made vulnerable to exposure - to be able to protect one's identity from automated enrollment in biometric recognition operations. The threat of discrimination that unequally affects marginalized communities persists and, in many ways, has not changed through a long history of racial and gendered discrimination in the US, but the technologies of control and regulation have. Biometric recognition enacts a negation of certain identities according to normative categories, prohibiting individuals from certain spaces and actively discriminating against certain groups. This change demands new and revised strategies of political agency.

Blas draws his central concept of "opacity" from the work of the Martiniquan philosopher and poet Édouard Glissant, who was active in the anti-colonial movements of the 1950s and 1960s. The art historian and novelist Teju Cole, in a New York Times article on photography and the representation of black skin, offers this insightful description of the term:

5 See also artist, Paolo Cirio, Obscurity (2016), inkjet prints, https://paoloci rio.net/work/obscurity/.

6 Zach Blas, "A Cage of Information, or, What is a Biometric Diagram?" in Documentary across Disciplines, ed. Erika Balsom and Hila Peleg (Cambridge, MA: MIT Press, 2016), 82. [80-90] 
Glissant defined it as a right to not have to be understood on others' terms, a right to be misunderstood if need be. The argument was rooted in linguistic considerations: It was a stance against certain expectations of transparency embedded in the French language. Glissant sought to defend the opacity, obscurity and inscrutability of Caribbean blacks and other marginalized peoples. External pressures insisted on everything being illuminated, simplified and explained. Clissant's response: No. ${ }^{7}$

Blas situates Glissant's concept of opacity within a contemporary, Information Age techno-political landscape and applies it to the language of data production - specifically to the surveilling of bodies and the transformation of physical embodiment into data. In the contemporary context, the "expectations of transparency" and an insistence on "everything being illuminated, simplified and explained" is enacted through the "forced visibility" of individuals through surveillance and biometric recognition technologies. Marginalized groups, such as people of color, immigrants and the LGBTQ community, are particularly vulnerable to state regulatory practices and standardization practices, which negate their identities through biometric recognition while, at the same time, forcing them to be, as Blas puts it, "informatically visible." ${ }^{8}$ Through the use of Glissant's concept of opacity, the right not to be seen takes on an ethical and concrete significance. In a nod to Glissant, Blas coins the term "informatic opacity," which he defines as

a refusal to visually cohere to digital surveillance and capture technologies' gaze. A theory and practice whose goal is maintaining the autonomous determination of alterity and difference [...] evading

7 Teju Cole, "A True Picture of Black Skin," The New York Times Magazine, February 18, 2015, https://www.nytimes.com/2015/02/22/magazine/a-true-pic ture-of-black-skin.html.

8 Zachary Marshall Blas, "Informatic Opacity: Biometric Facial Recognition and the Aesthetics and Politics of Defacement," (PhD diss., Duke University, 2014), iv. 
the quantification, standardization, and regulation of identity imposed by biometrics and the state. ${ }^{9}$

In this way, informatic opacity can function as both a tactic and a material condition. It can serve as a tool of political activism, within the discourses of identity politics, in enabling people to oppose a politics of identification. The right not to be visible equals a right to self-determination, to be able to "self-generate" an identity that would otherwise be negated through processes of biometric recognition.

\section{Facial Weaponization Suite (2011-2014)}

Blas produces a materialization of "informatic opacity" in his masks and through a vehicle of "excess." Blas describes how he constructs the masks from the faces of multiple participants who attend workshops he runs in collective art spaces. The participants at the workshop agree to be scanned using a Kinect 3D scanning device, which yields data relating to each person's face. Blas then collaborates with a modeler to generate a mold of a mask from the compiled facial data from all the participants. Blas states that this facial data is "not averaged" but rather compiled into a formless shape. He describes how this results in an "amorphous mask that resembles only abstract surfaces," making it "biometrically unrecognizable." 10 mold is produced through a process of CNC milling, that is, "computer numerical controlled" machining, which relies on programmed code to determine the movements that create the mold. This method allows for a high degree of precision in movement. CNC milling utilizes a rotating cylindrical cutting tool; the piece is moved across the milling tool in different directions, making it possible to create the amorphous, customized shapes of Blas's masks. This mold is then used to vacuum form multiple masks.

9 Ibid.

10 Blas, Informatic Opacity, 78. 
Blas has exhibited both the masks themselves and highly stylized studio color photographs of the masks. The masks themselves are glossy, highly reflective and brilliant in color. The monochrome colors of the masks are significant. Most of Blas's masks are simply titled Mask, with the date and location of the mask's production, but the colors allude to specific cultural contexts and symbolize the normative categories that these masks work to obfuscate. For example, the creation of a blue mask came out of a workshop Blas held that was attended by artists, intellectuals, curators, activists and technologists on the subject of feminist theory. The workshop covered issues of visibility, recognition and concealment, and a particular issue arose concerning the use of the veil by Muslim women and how this "complicates western feminism's investment in visibility politics." They discussed a particular incident that had become a kind of inflection point in relation to these issues. The incident occurred in 2011 during the uprisings in Cairo, Egypt, and is referred to as the "blue bra" incident. A woman wearing an abaya was participating in the demonstrations in Tahrir Square, and she was brutally beaten by Egyptian soldiers. As she was dragged off, her clothes were ripped away, revealing a blue bra (figure 22). ${ }^{12}$ The image of the blue bra became a feminist rallying symbol for Egyptian women protesting oppression. ${ }^{13}$ In reference to this incident, the color blue was chosen for the mask that was produced following the workshop (figure 23). The form and shape of the mask also recalls the veil worn by Muslim women. The use of blue in Blas's mask symbolizes the issues that sit at the intersections of feminist resistance in the cultural context of the Middle East, the wearing of the veil and investment in both concealment and exposure.

11 Ibid., 163.

12 Kainaz Amaria, "The 'Girl in the Blue Bra," NPR, December 21, 2011, https://www.npr.org/sections/pictureshow/2011/12/21/144098384/thegirl-in-the-blue-bra.

13 Blas, "Informatic Opacity," 162. 
Figure 22: Egyptian army soldiers arrest a female protester during clashes at Tahrir Square in Cairo on Dec. 17. () Stringer/Reuters/Landov

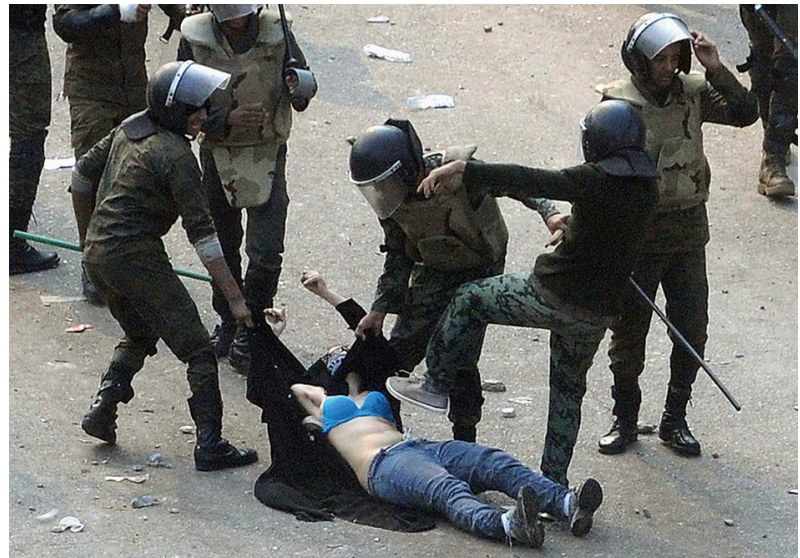

Figure 23: Zach Blas, Mask- November 20, 2013, New York, NY, Facial Weaponization Suite, Photo by Christopher O'Leary. Image courtesy of the artist

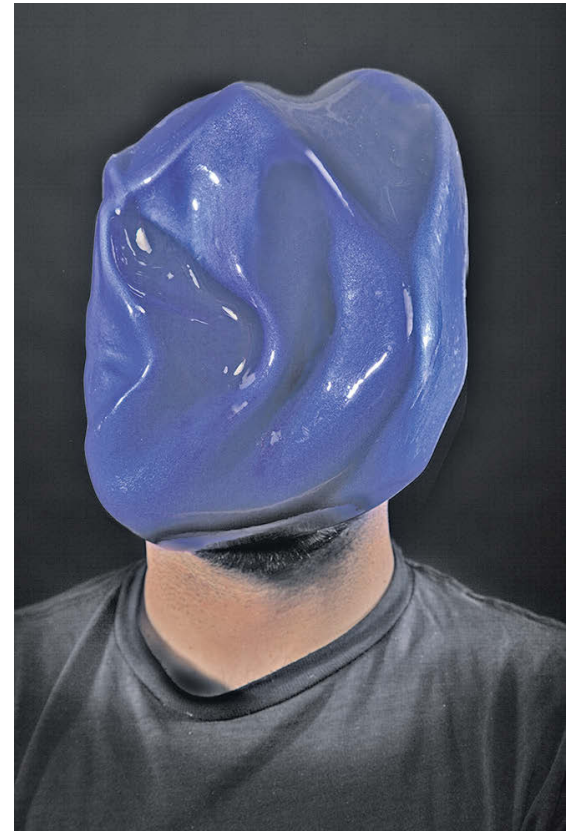


Figure 24: Zach Blas, Fag Face, Facial Weaponization Communiqué: Fag Face, video still (2012) Image courtesy of the artist

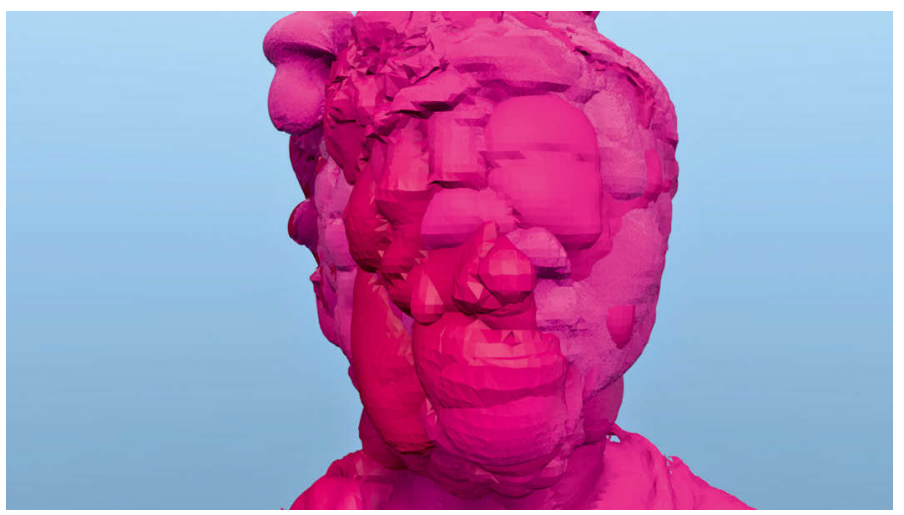

The first mask Blas created in the suite is one of the few to be given a name other than "mask." It is titled Fag Face (2012) (figure 24). (The figure shows an image of a virtual model of the mask, taken as a video still from Blas's short film "Facial Weaponization Communiqué: Fag Face.") In constructing this mask, Blas had in mind certain scientific studies, such as one conducted at Tufts University, on the recognition of homosexuality. These studies tested for an ability to identify homosexuality through a rapid recognition exercise using photographs of men's faces. There has since been much controversy over similar research into the machinic recognition of homosexuality, in particular studies conducted at Stanford University by Michal Kosinski and Yilun Wang. ${ }^{14}$ Blas zeros in on the most pertinent question one might have upon learning of the existence of these studies, which is: why? To what ultimate end will these tests contribute? Of all the possible purposes to which these tests could contribute, the primary and most obvious is the control and regulation of homosexuals through the utilization of the technological acts of sorting and categorizing. Blas constructed the mask Fag Face as a direct response to the scientific studies at Tufts on rapid facial recognition of sexual orientation. Like the other masks in the suite, it was generated by scanning multiple faces, but in this case specif-

14 See "Keeping a straight face," The Economist, September 9, 2017, 67-68. 
ically gay men's faces. The resulting physical mask is a candy-pink blob of gloss (figure 25). Although constructed from data about the faces of gay men, this mask obscures the identity of each individual face and that of the wearer of the mask. In response to the Tufts test, the mask accelerates the "rapid recognition" aspect by expressing the faces of multiple gay men at once. Through both the elucidative material of the mask and its instantaneous projection of a multitude of facial forms, it renders a kind of play between full-on visibility and concealment.

Figure 25: Zach Blas, Fag Face Mask - October 20, 2012, Los Angeles, CA. Facial Weaponization Suite. Photo by Christopher O'Leary. Image courtesy of the artist

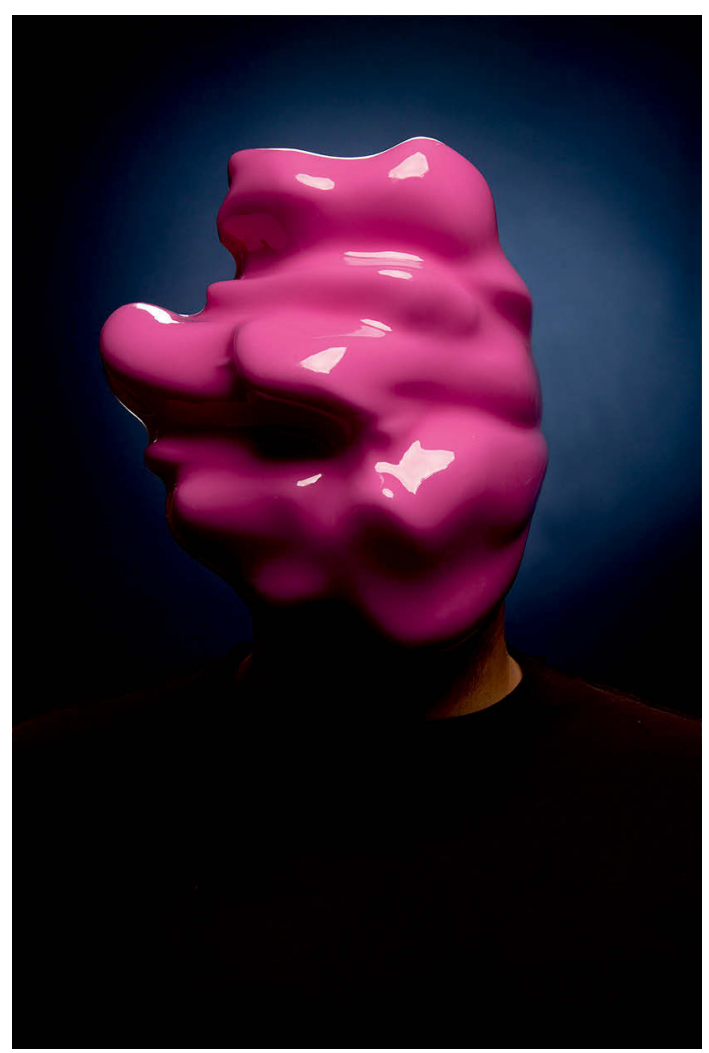


On another level, Blas's work is performative. After a mask is completed, the workshop participants create a masked public performance that highlights the inequalities inherent in biometric facial recognition use. Blas has chosen to hold these performances in areas of particular relevance to the issue of facial recognition. For example, one performance, titled Procession of Biometric Sorrows, was held at the US-Mexico border on June 5, 2014. (figure 26). It called attention to the immense amount of biometric data that is gathered at borders and in particular at the US-Mexico border. Blas notes that a central subject of discussion in the workshop preceding this performance was the fact that the Mexican government had recently introduced biometric identification cards for children. ${ }^{15}$

Figure 26: Zach Blas, Facial Weaponization Suite: Procession of Biometric Sorrows, Museo Universitario Arte Contemporáneo (MUAC) Mexico City, Mexico (5 June 2014). Photo by Orestes Montero Cruz. Image courtesy of the artist

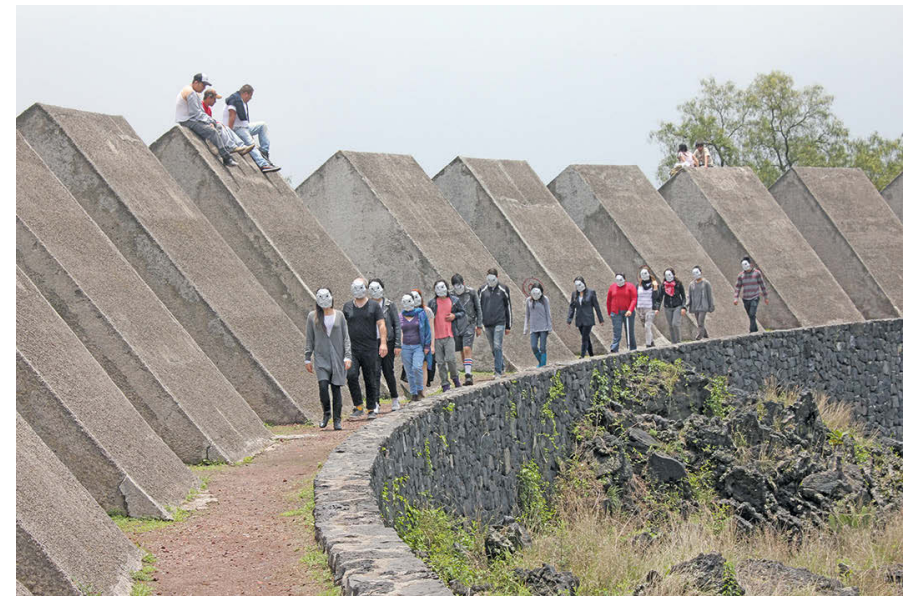

15 This issue of biometric identification cards for children foreshadows the more recent incidents of mistreatment of Mexican children at the US-Mexico border. Children have been separated from their parents and detained by US authorities in camps at the border, a practice that has been understood as a gross abuse of power by the Trump administration. 


\section{A Collective Excess}

These performances, as well as the creation of the masks themselves, highlight Blas's collectivist strategy. In drawing on this strategy, he references contemporary political movements that rely on a social collectivity to confront the widespread abuse of institutional and governance powers, such as the Occupy movement, Pussy Riot and the Zapatista movement in Mexico. These particular movements rely on a strategy of masking or facial concealment: Occupy with the use of the Guy Fawkes mask, the pink baklavas of Pussy Riot and the Zapatistas' bandanas, which conceal the lower half of the face. Blas's reference to a "weaponization" of the face is an acknowledgment of these movements; what he calls the "power of the collective face" merges a socio-political apparatus of collectivity with faciality. $\mathrm{He}$ is pinpointing the face as a site of politics. In these movements, the face is recreated; it is reconstructable and interchangeable, rather than being a source of recognition and thereby a means of regulation by the state. Removing the recognizable features of the face, the members of these movements become a faceless threat to the asymmetrical systems of power that they confront. In this context, facelessness is a source of power. The use of masks in Blas's performances draws on a historical use in the context of the carnival, where members of the populace were able to speak truth to power and where social and political hierarchies were inverted through satire. ${ }^{16}$ In this context, masked performances play with the distinction between what is seen and unseen. They at once make the wearer highly visible, giving their performer a platform, and at the same time erase individual identity. This allows for the wearer of the mask, and so the speaker of truth, to be anonymous.

Blas's masks are constructed from the forms of many faces, and as such they merge the individual wearer of a mask with the masses. In this way, for me, the masks recall Philip K. Dick's "scramble suit" in his psychotropic sci-fi novel A Scanner Darkly. In Dick's novel, the

16 The use of masks in these sorts of contexts spans many different cultures. See, for example, the use of "tal" masks in Korea; the etymology of the word derives from the Chinese character meaning "to be free." 
"scramble suit" is worn by narcotics officers, and thus is a technology of policing. He writes:

The scramble suit was an invention of the Bell laboratories, conjured up by accident by an employee named S. A. Powers [...] Basically, his design consisted of a multifaceted quartz lens hooked up to a million and a half physiognomic fraction-representations of various people: men and women, children, with every variant encoded and then projected outward in all directions equally onto a superthin shroudlike membrane large enough to fit around an average human.

As the computer looped through its banks, it projected every conceivable eye color, hair color, shape and type of nose, formation of teeth, configuration of facial bone - the entire shroudlike membrane took on whatever physical characteristics were projected at any nanosecond, then switched to the next [...]

In any case, the wearer of a scramble suit was Everyman and in every combination (up to combinations of a million and a half sub-bits) during the course of each hour. Hence, any description of him - or her-was meaningless. ${ }^{17}$

The description of the "scramble suit," with the "everyman" projection of every face obscuring the wearer's identity, could be a description of Blas's masks. Blas's masks, like the "scramble suit," present a representation of multiple identities as a strategy of camouflage. It is interesting to think about the use of "Everyman" in Dick's scramble suit in relation to Blas's strategy of "collectivism." Both erase any characteristic features through an excess of features. In other words, in showing too much one cannot see anything at all. This is a tool used by certain artists in the Information Age $^{18}$ to confound systems of surveillance and control. It is a tool that makes

17 Philip K. Dick, A Scanner Darkly (New York: Vintage Books, 1991), 22-23.

18 See work of artist Hasan Elahi. Hasan Elahi website, accessed June 3, 2019, http://elahi.umd.edu and Karen Kedmey, "Hasan Elahi vs. The FBI: The Art 
use of the original material of surveillance, that is, the information itself, and overloads the system with it. Like Dick's "scramble suit," which "projected every conceivable eye color, hair color, shape and type of nose, formation of teeth," Blas's masks use the distinctive characteristics and endless variability of the face as information, before combining it in order to produce indistinction. Blas's masks project this mutability as an opaque form all at once, however, rather than in nanoseconds. Instead of a multifaceted quartz lens, the material of Blas's masks is opaque and reflects only light, rather than faces, from off of its glossy surface. This difference also reveals that Blas's masks play with the notion of the surface. What can be seen in his masks is only surface, and because that surface is highly glossed and vividly colored, the observer's attention is drawn to it rather than to anything underneath. This mirrors the fact that facial recognition technologies can only recognize surface qualities and that surface can be dressed up however an individual chooses. In Dick's novel, this is used as a method of concealing the identities of members of the police. In Blas's work, it is used as an aesthetico-political tool to conceal the identity of the individual from dominant systems of population control. Instead of being a tool of policing, Blas's masks are a weapon for the proletariat. As the title of Blas's project makes clear, when used as a tool of resistance, the collective is the weapon.

In their opposition to standardization and in their formlessness, Blas's masks exhibit a strategy of excess. Blas has described his masks as a "collective excess, that exceeds the boundaries of the individual."19 In a written piece titled "Fag Face," Blas has explained this excess further, using terms that are physical, subjective, bodily and embodied. Excess is utilized here as a defiant response to the inherent violence in the use of the term "fag" as a derogatory label for gay men:

of Self-Surveillance," Artsy, May 27, 2016, https://www.artsy.net/article/ artsy-hasan-elahi-vs-the-fbi-the-art-of-self-surveillance.

19 Blas, "Informatic Opacity," 79. 
Fag face captures me into an identity that is not my own, a grid that legislates me.

How do I escape this face? How do I desire to escape this gridding of my head? How can I open, make into a mystery, liberate my fag face?

Force the face forward. Bring the face to the limit of these grids, so that it can de-code its boundaries, break them open, to enter again into the swarming chaos of matter that resists recognition.

If fag fucking is what fag face visualizes to the other, push this further.

Accumulate cum so that your face becomes a volatile liquid surface with no eyes, nose, or mouth; keep the smell from rimming so that your face and ass are irreducible; let the pubic hair gather into different consistencies of stickiness; wipe the shit left on your fingers under your hidden, cum-filled eyes like war paint. Transform your face into a hypertrophized state of fag-ness. Let these new excesses dissolve readability. Let your fag face configure with these materials into that which is not identifiable

Once 1000 cocks have cum on my head and 1000 asses have wiped their shit and sweat there, try to tell me what my face is. ${ }^{20}$

Like his masks, Blas's text opposes the standardization mechanisms of facial recognition through a tactic and materialization of opacity. In this case, opacity comes in the form of excess, an excess expression of that which underlies homophobia. Opposing the mechanized and automated processes of a biometric operation, this text, as lyrical poem, is unapologetically human in all its corporeal glory, both in flesh and liquid form, and in its tenor of punk-rock revolt. The text references the bodily sense that cannot be measured,

20 Zach Blas, "Fag Face," Recaps Magazine, accessed May 3, 2019, http://re capsmagazine.com/review/fag-face-by-zach-blas/. 
including desire. The text is not only a statement of resistance to an operation of facial recognition but confronts the individuation of recognition and the regulation of gay male sexuality with an excess of it: I will meet your homophobia with my cock - 1000, to be precise. Strength in numbers. Through Blas's textual work and in his production of masks, he inverts an essential dilemma and demand to which biometric technology is so often called upon to respond the problem of volume, or specifically the problem of ascertaining identity from a massive amount of information. Blas utilizes the problem of volume and transforms it into an aesthetic, solidifies it in sculptural form as a materialization of opacity.

Blas's artwork is particularly significant when considered in relation to the eigenface algorithm and the composite form analyzed in the previous section. Through his work, the composite form itself is made operational. His approach to designing the masks references a history of facial recognition techniques, as he makes clear in his dissertation. ${ }^{21}$ Blas directly references the work of Francis Galton and his composite portraits. He describes his masks and the corresponding processes of "digital collectivization" as the "antithesis" to Galton's composites of criminals and production of types. ${ }^{22} \mathrm{He}$ states: "In contrast, the collectivizing process in Facial Weaponization Suite reveals that Galton's compositing method can be employed to arrive at the exact opposite of his intentions [...] it can also generate a collective excess that exceeds the boundaries of the individual. ${ }^{123}$ This brings to mind a passage from Galton's description of his composite portraiture practice: "No statistician dreams of combining objects into the same generic group that do not cluster towards a common centre; no more should we attempt to compose generic portraits out of heterogeneous elements, for if we do so the result is monstrous and meaningless." ${ }^{24}$ With his sculptural composites, Blas injects meaning into the "monstrous." Although Blas sees his work as the antithesis of Galton's production of a type, his

21 Blas, "Informatic Opacity."

22 Ibid., 79.

23 Ibid.

24 Galton, Inquiries, 230. 
practice nonetheless involves, I argue, a similar process of production and creates a similar structure to Galton's composite. In the bringing together of people under categories of identity such as "gay men," Blas also produces a composite. It is this similarity of structure that makes Blas's work a fascinating study in this regard: Blas then takes the composite form and produces something that is the opposite of a "type." Blas's rejection of Galton's type through his masks and through what he calls the "collective excess that exceeds the boundaries of the individual" results in a sculptural formation of the Galton composite.

The masks visualize something very similar to Wittgenstein's concept of "aspect perception." Both "aspect perception" and "collective excess" turn the aesthetic focus of the composite form on to the mutability and fluidity of forms. They both reveal a kind of relationship between disparate forms. The notion of collective excess operationalizes the notion of aspect perception in contemporary politics. Blas's masks can be understood, in this way, as solidifying a mutability of forms and activating the composite precisely to resist an automated (and reductive) recognition process. To return to the topic of eigenface, we might imagine Blas's masks as what would happen were one to produce an eigenvector in physical form and then reflect it back into the eigenface algorithm. In this way, I see Blas as taking the output data from an algorithm and projecting it on to its front end. It is like an algorithmic mirror in which the algorithm cannot recognize itself or what it has produced. It is as if the algorithm cannot read its own form of representation.

\section{Concluding Remarks}

Blas's Facial Weaponization Suite is not practical; it does not offer a strategy for actual informatic opacity in the face of non-consensual biometric enrollment - unless, that is, we were all to walk around with masks over our faces in our daily lives. Yet what I find most important about his piece and the many forms it has taken - workshop, performance, short film, text and masks - is that they all point toward the production of another form of subjectivity, one that is the 
result of a "collective excess." Discussing Facial Weaponization Suite, Blas has said that the masks "articulate a presence." ${ }^{25}$ The presence is formed through a collective. In Blas's strategy of negating the process of biometric recognition - albeit in a symbolic fashion, through performativity and masking - there is the formation of a kind of collective subjectivity, one that is not defined by individuation. This has far-reaching implications for our understanding of the parameters of future forms of political, ethical and social organization. Blas's masks are a conglomerate of a multiplicity of angles and perspectives from different faces, depicting a kind of endless variation. Their construction draws on the concepts, strategies and discourses of queer theory, feminist critical theory and post-colonial theory. They propose an alternative representational mechanism to the one found in AFR systems, a mechanism that captures a mutable human form. The result is a representation of a subjectivity that is manifold and self-generated. The representation found in Blas's work is of a collective figure, reflecting a collective subjectivity.

25 Valentine, "Weaponizing Our Faces." 



\section{Chapter 8: An Algorithmic Ready-made: Trevor Paglen, Adversarially Evolved Hallucination and Eigenface (Even The Dead Are Not Safe)}

\section{Background: Notions of Transparency}

While Blas's work references a notion of opacity, the work of the artist Trevor Paglen conjures up its opposite, transparency. Much of Paglen's work involves the production of images in environments and milieus where there has been a conscious effort to suppress images. Paglen's artwork has exposed the nature of opacity in covert military and political agencies, such as the NSA and the CIA, revealing a surrealist character to their agendas and the physical structures through which they operate, forcing a kind of transparency into their workings. His recent works concern the topic of machine vision and its increasing prevalence in society. He approaches the topic of machine vision as he does his other subject matter, exposing the hidden and obscure processes through which it operates and creating spaces of transparency and visuality. What particularly interests Paglen about machine vision is the growing economy of images, produced by and for machines, that is evolving and, for the most part, going unseen by the human audience. His inquiry acknowledges the influence of machine vision in bringing about a transformation in contemporary visual culture as a whole, where increasingly, he argues, human vision is an exception to the 
rule. ${ }^{1}$ And yet, as Paglen argues, these machine vision images play an increasingly large role in human interactions, with "their functions changing from representation and mediation, to activations, operations, and enforcement," thus making it necessary for us to scrutinize not only the images but the processes through which they enact a form of vision. ${ }^{2}$

In his attempts to reveal the inner workings of forms of machine vision, Paglen produced a series of images using different artificial intelligence (AI) systems of machine vision. These works culminated in an exhibition at Metro Pictures Gallery called A Study of Invisible Images. ${ }^{3}$ Some of the works included in this exhibition appropriate the actual processes of algorithms that have been designed to enact different operations of machine vision. Two such works will be discussed here: a series titled Adversarially Evolved Hallucination (2017) and a series of works made with the eigenface algorithm titled Eigenface (Even The Dead Are Not Safe) (2017). ${ }^{4}$ In both of these works, Paglen approaches the technology of image recognition systems (with the latter dealing directly with a facial recognition system) and provides a kind of visual dissection of their algorithmic processes.

In producing these works, Paglen is part of a growing movement of artists using code to make art. I would describe Paglen's artistic approach to image and face recognition technology as internal; that is, he uses the algorithmic processes themselves as material for the

1 Trevor Paglen, "Invisible Images (Your Pictures Are looking At You)," The New Inquiry, December 8, 2016, https://thenewinquiry.com/invisible-images-your-pictures-are-looking-at-you/.

2 Ibid.

3 Trevor Paglen, A Study of Invisible Images, exhibition, September 8-October 21, 2017, Metro Pictures Gallery, New York, NY.

4 Paglen included other works in this exhibition that refer directly to different facial recognition systems, including It Began as a Military Exhibit and Machine-Readable Hito. I chose the two works discussed here because of their connection and relevance to the overall analysis. Another of Paglen's works that bears on facial recognition (specifically automated facial-behavioral analysis) is his piece Sight Machine, performance, January 14, 2017, San Francisco, CA. 
production of images. In this way, his artwork is less a confrontation with the technology's front-end operations and more an engagement with the internal workings of how the technology enacts recognition. This approach in turn, alters the production and operation of the algorithm itself. This contrasts with Blas's work, where our attention is largely drawn toward a confrontation between the recognition device and the embodied subject under surveillance. In presenting images that express how the internal workings of AI systems see, Paglen's work has a way of being pedagogical, teaching an observer of his images about how an algorithm sees. In the absence of further explanation, this aspect can make his work somewhat opaque and hard to penetrate.

One of the strengths of Paglen's work is its ability to put technological processes into dialogue with discourses in art history and political philosophy. In doing so, his works not only expose but also actively reimagine the technological processes at issue and hold out the promise of a reinterpretation of the possibilities of the technology's operations. Paglen finds unintentional affinities between abstract art and linear classifiers - the archetypes used to train algorithms how to recognize objects. I see Paglen's use of algorithmic processes to produce images within a cultural context, in fact, as a reference to another art-historical practice, that of the ready-made, a method of art production involving found but modified objects/ products, first used by Marcel Duchamp, who presented manufactured objects as objects of art. An aspect of the ready-made is the use of ubiquitous, everyday objects. In the contemporary world, algorithms have become one such everyday object; although they are immaterial and their presence undetectable, they are ubiquitous at all levels and areas of interaction. Paglen's appropriation of image recognition algorithms treats them as an immaterial ready-made: that is, he uses preprogrammed or "found" algorithms and modifies their output, positioning this output within a discourse of art production and within various conceptual frameworks. In producing an "algorithmic ready-made," Paglen takes the everyday object of the algorithm and merges its technical processes with the conceptual. 


\section{Adversarially Evolved Hallucination (2017)}

Paglen produced the series of works titled Adversarially Evolved Hallucination during a residency at Stanford University. In referring to these images as "hallucinations" generated from machine-vision processes, Paglen characterizes them as departing from reality - as though we are about to see what machines see while on acid. There is, he is saying, an illusory quality to the machine-vision processes usually deemed objective, scientific and engaged in definitive operations. The word "adversarial" in the title of the series refers specifically to images that are developed by computer scientists to trick image recognition systems. These "adversarial images" are developed to exploit weaknesses in the recognition algorithm, making them see things that are not there. The purpose of these images is to better train the algorithm - a kind of exercise of productive technical failure. These adversarial representations eventually get incorporated into the training sets used by the algorithm in machine learning. With the help of a small team of developers, Paglen customized the software used for recognition and developed software suites. He used two separate algorithmic processes: one that taught the machine to recognize objects through training sets and the other that was used to generate adversarial images based on the same training set. Paglen referred to the secondary algorithm as the "Generator," while the first he called the "Discriminator." $\mathrm{Pa}$ glen designed these two algorithms to engage in a dialogue with each other: to play a game in which the second algorithm attempted to fool the first through the production of adversarial images. $\mathrm{Pa}$ glen explains:

The two Als go back and forth thousands or millions of times, until the Generator has learned how to make images that can reliably fool the Discriminator. The images that come out of this process are called Hallucinations. Together, the Als have evolved an image that

5 Metro Pictures, Trevor Paglen: A Study of Invisible Images, (New York: Metro Pictures, 2017), 24.

6 Ibid. 
is entirely synthetic and has no referent in reality, but that the pair of Als believe are examples of things they've been trained to see. ${ }^{7}$

The resulting images are surreal, pixelated forms that emerge in vivid color. The game that the two AIs play is not only a way of exploiting the weaknesses and parameters of algorithmic learning; it is also a game that plays with the idea of recognition itself. How does the AI know something as it is? What can be recognizable? In this algorithmic dialogue, Paglen toys with the relationship between visual form and knowledge. A key aspect of these works is found in their titles, which are taken from the categories of training set images used by the algorithms. Instead of the phrase "training set," Paglen uses the term "corpus," bringing attention to the role of training sets as a body of knowledge. The corpuses that are referred to in the titles include "Omens and Portents," "Monsters of Capitalism," "American Predators" and "The Aftermath of the First Smart War." These categories organize the types of objects being recognized within Paglen's software suite, and they clearly differ from the actual categories of training sets used in machine image recognition. Instead, they reflect concepts that act as metaphors, describing the wider socio-political contexts in which the technology is used. The images that result from this algorithmic game of recognition render these metaphors visible. For example, one image, Highway of Death (Corpus: The Aftermath of the First Smart War) (figure 27), references the militaristic context of the use of image recognition. The image is a surrealistic rendering of a desert battlefield with no humans present. What is present in the image is a shift in replacing the categories of training sets to recognize objects or people to the recognition of the cultural contexts of its operation. Through Paglen's production of adversarial images, he is experimenting with training an algorithm (and the human observer of the generated image) to not only see objects but also to see concepts that shed light on the wider cultural contexts in which these technologies intervene.

7 Ibid. 
Figure 27: Trevor Paglen, "Highway of Death (Corpus: The Aftermath of the First Smart War)" Adversarially Evolved Hallucination, 2017. dye sublimation metal print. Image courtesy of the artist

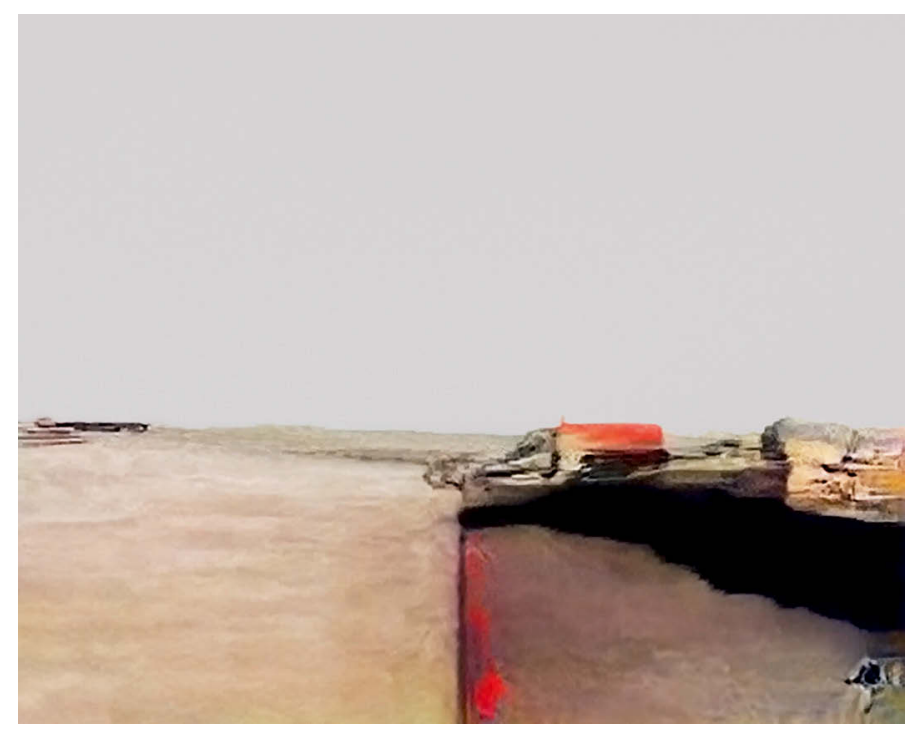

For Paglen, the importance of developing a vocabulary through metaphor connects back, I think, to a previous series of his, Symbology (2006), in which he collected material paraphernalia relating to black ops and covert activities of the CIA, including fabric patches, designed by members of covert units, that are embroidered with coded insignia, symbols such as dragons, arrows, animals, planets and phrases such as "We Own the Night." The character of the metaphoric forms and titles found in the images of Adversarial Hallucinations may be seen as mirroring these ominous symbols and phrases. Both reflect a world that would otherwise not be seen, and once exhibited become objects of conceptual inquiry. Paglen describes the patches used in Symbology as follows: "A part of the military's everyday culture [...] If you could begin to learn its grammar, you could get a glimpse into the secret world itself." ${ }^{8}$ This description

8 Trevor Paglen, I Could Tell You But Then You Would Have to Be Destroyed By Me: Emblems From the Pentagon's Black World (Brooklyn: Melville House, 2010), 4, 5. 
can be extended, I think, to his approach in Adversarial Hallucinations, which develops a visual vocabulary of algorithmic processes, building on a taxonomy of corpuses, providing a grammar to see how machines see within the contexts in which they operate.

Some of the titles of these corpuses refer to literary metaphors. Through the corpus titles, Paglen refers to a conceptual taxonomy based in literature and philosophy. As I have mentioned, one of the corpuses references the allegorical monsters of capital, ${ }^{9}$ that is, monsters that have acted as literary metaphors for capitalism. One in particular, takes the form of a face - Paglen's piece Vampire (Corpus: Monsters of Capitalism) (figure 28). The image is pretty terrifying. It is of a face that appears to be constructed from melting pixels. It contains all the facial landmarks: eyes, nose, cheeks and mouth. Yet these landmarks are composited together from disparate sources and fragments of light and shadow, creating a kind of digital collage work. The left eye resembles a photograph of an actual eye seemingly cut out from a magazine, and it peers out, furtively, from a dark curtain of velvet blur. From this eye, bluish veins appear to drip down. The other eye, in contrast, is constituted by a dark swirl resembling a wilting red carnation. A jagged lightning bolt parts light and shadow across the face and acts to form the nose. In place of a mouth is a kind of striated, conglomerate of blood-red, pixelated ooze. Much of the face is hidden in shadowless depths of darkness. In the areas of light, specifically in the forehead, one can detect a painterly effect of the multiple layers of digital imagery that constitute this composited face. The predominance of a Dada-like palette of black, white and red contributes to the stark nature of this vampire face. The more realistic left eye is the only part of the face that is a recognizable, complete form. Without that eye one could hardly see a face in this image. That eye is thus both an organizing feature and also the source of the image's terror. For, in its fully realized form, it is what allows the vampire/monster in the image to look back at the observer, and as an observer we feel very much under its mono-gaze.

9 For more on this subject see David McNally, Monsters of the Market: Zombies, Vampires, Global Capitalism (Chicago, IL: Haymarket Books, 2012). 
Figure 28: Trevor Paglen, "Vampire (Corpus: Monsters of Capitalism)" Adversarially Evolved Hallucination, 2017. dye sublimation metal print. Image courtesy of the artist

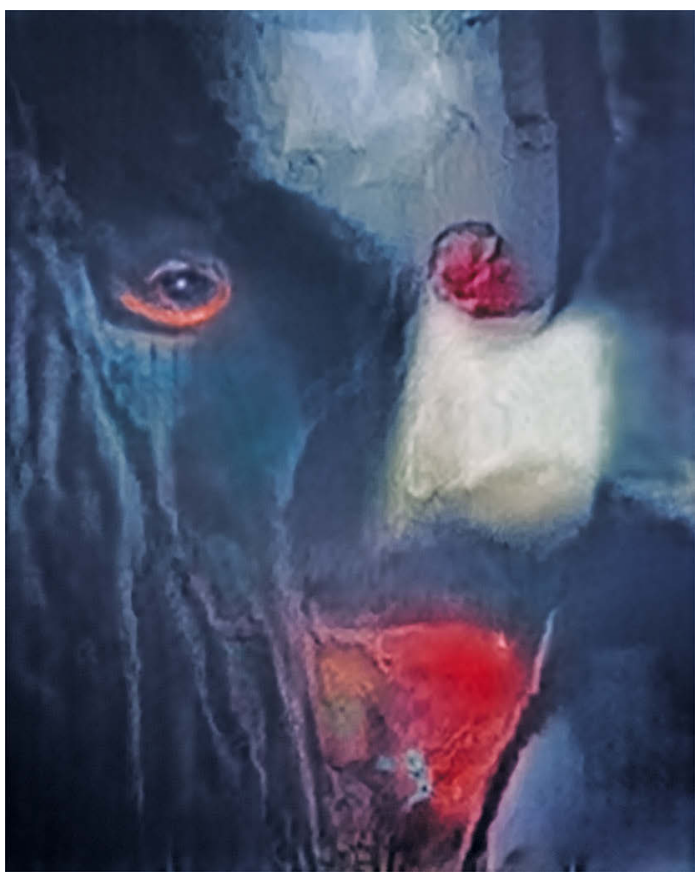

Paglen has described how he thinks of AI itself as a monster of capitalism. The subject of the image in Vampire (Corpus, Monsters of Capitalism) alludes to Karl Marx's vampire of dead labor: "Capital is dead labour, that, vampire-like, only lives by sucking living labour, and lives the more, the more labour it sucks." ${ }^{\text {"O }}$ As such, the image references a wider context in which market forces drive image-recognition algorithms, and AI in general, to be deployed in order to replace human labor. In his allusion to Marx and with his depiction of a vampire as one particular monster of capitalism, his work may be seen as a critique of the forces and agendas behind the technology's development and use - politically, commercially and militarily.

10 Karl Marx, Capital: A Critique of Political Economy, vol. 1, trans. Samuel Moore and Edward Aveling, (Moscow: Progress Publishers, 1887), 163. 
His piece visualizes the "face" of this "monster of capitalism," and as such it stands out in the context of this thesis on facial recognition. For this is another construction of a portrait by an algorithm, yet it is one based on and born entirely from a concept. In this way, $\mathrm{Pa}$ glen also says something about faces as conceptual constructs. This face was constructed, as were the other images in the series, from a training set of thousands, if not millions, of collected images - in this case, various images of vampires.

Figure 29: Trevor Paglen, training set images for "Vampire (Corpus: Monsters of Capitalism)" Adversarially Evolved Hallucination, 2017. Image courtesy of the artist

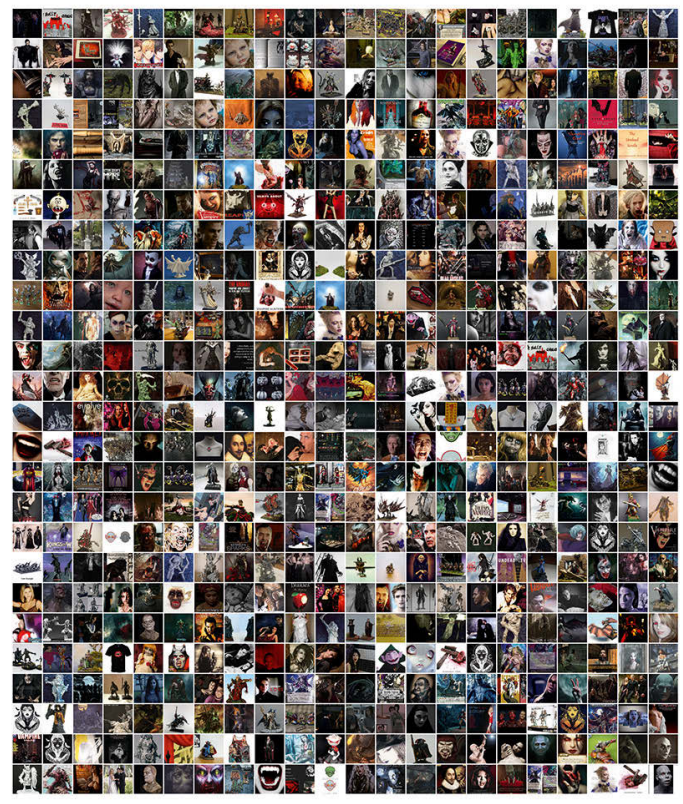

Figure 29 shows a sample of the training library, revealing a wide variety of cultural imaginations of vampires, ranging from the beloved Count von Count Muppet from Sesame Street, to photographs of infant vampires, to depictions of vampiric motifs in fashion spreads and medieval paintings. 
In focusing on the initial training-set phase of the recognition process, Paglen problematizes a central issue with automated recognition through AI: the way algorithms learn through training sets and, as such, fix meaning in the physical world. Paglen describes the training-set process as follows:

[t]his all takes place largely for the most part imperceptibly. Assignations of meanings, of gender, race, species, marketability, and criminality, are done both autonomously and invisibly; we cannot see how we are being named, much less audit the processes through which that naming happens. ${ }^{11}$

In short, as Paglen states, "he who controls the training sets controls the meanings of images." 12 The naming of the categories of training sets ultimately determines the kinds of knowledge an algorithm can (and cannot) produce. Playing with the naming of the corpuses not only allows Paglen to introduce metaphors that describe the contexts of the technology's use but also exposes a loosening in the interplay of signs, both visual and linguistic, that is occurring through the technology. The algorithmic game that produces the images in Adversarially Evolved Hallucination bears on notions of recognition, creating a space of negotiation between what is known and what can be seen/recognized. Through the use of language and metaphor, Paglen presents not only the socio-political contexts of the technology but also to suggest that human culture and imagination can intervene in and counter the automated naming processes that ascribe meaning, processes that otherwise remain hidden within the internal, circulatory processes of the technology. Through language and visual metaphor, Paglen injects cultural meaning and context into machine-vision processes. In doing this, he also highlights a central issue of A.I.: that cultural meaning and context are two major blind spots of machine-vision processes.

11 Trevor Paglen, "Machine Realism," in I Was Raised on the Internet, ed. Omar Kholeif (Munich: Prestel, 2018), 118.

12 Ibid, 116. 


\section{Eigenface (Even the Dead Are Not Safe) (2017)}

Paglen elevates the eigenface image to the level of an artwork in his series Eigenface (Even the Dead Are Not Safe). His eigenface portraits are not formed from an average of multiple faces but rather from a compilation of multiple facial images of the same subject. For his subjects, he chose philosophical and literary figures such as Franz Fanon, Samuel Beckett and Simone Weil. (He also made one of the actress, Winona Ryder.) His process of making these eigenface images involved projecting eigenvectors made of each individual subject on to face spaces of other people and mathematically calculating the differences between them. In this way, Paglen reenacted the usual recognition process of the eigenface method, but instead of projecting a captured image of an unknown face, he projected an eigenvector of a known subject, and the resulting differences, which are normally translated into code and stored in a database as representing the identity of the subject, he instead visualized in these images. Because of the way it depicts the features that distinguish the subject from everyone else, Paglen refers to the resulting image as a "faceprint." ${ }^{13}$

As an artwork, Paglen's eigenface portraits contrast with the eigenface images that normally result from the operation of its algorithm, examples of which I have discussed in previous chapters. In the first instance, these portraits are in color. Rather than the usual gray-scale images, these portraits have a washed-out, cold, sepia tone. As they are compilations of multiple images of a single subject, these portraits also clearly portray that subject, albeit with the blur characteristic of eigenface images. The aesthetic of the mathematically abstracted blur, together with the washed-out tones, produces a ghostly rendering of these subjects. The image of Franz Fanon, titled Fanon (Even the Dead Are Not Safe) (figure 30), is, I find, the most striking of these portraits. It is a haunting portrayal of the ghost of Fanon, with his face veiled in a chalky pallor and the color of his skin only retained around the shadows of his eyes and lips and along the edges of his face.

13 Metro Pictures, Trevor Paglen: A Study of Invisible Images, 9. 
Figure 30: Trevor Paglen "Fanon" Eigenface (Even the Dead Are Not Safe), 2017. dye sublimation metal print. Image courtesy of the artist

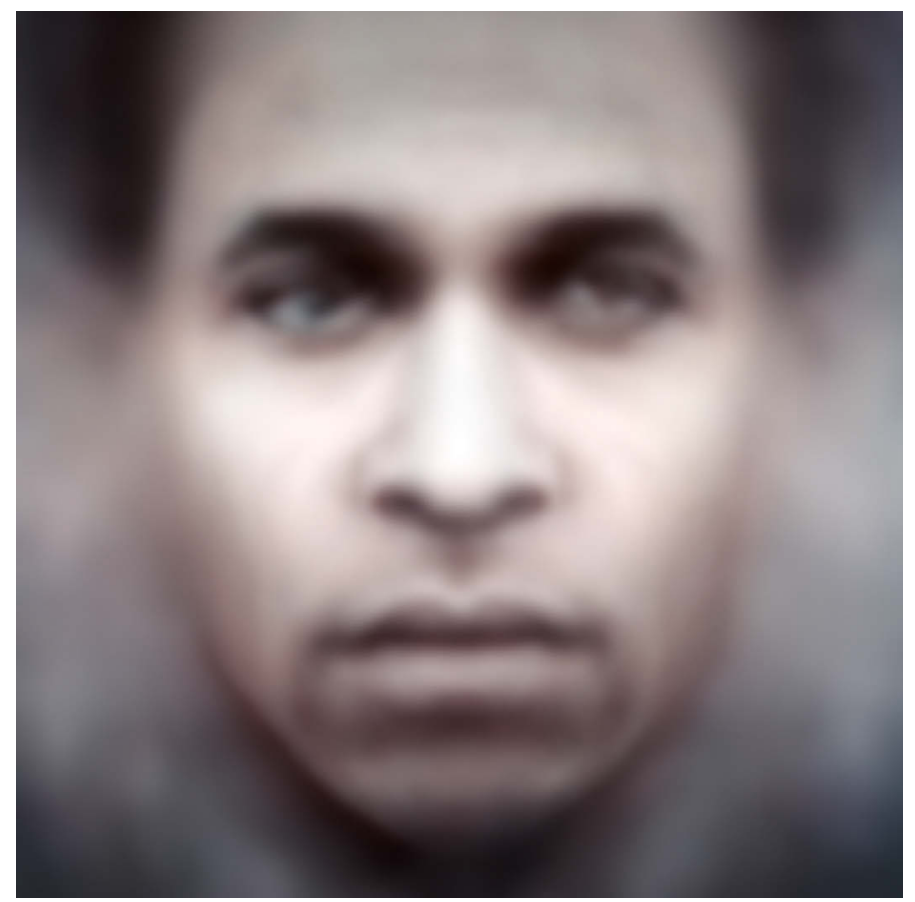

There is an expression of urgency in his return gaze. Upon staring into his eyes, one is unsettled by the fact that the color differs slightly in each - one with a bluish hue and the other brown. Fanon once stated: "We who come from the Antilles know one thing only too well: Blue eyes, the people say, frighten the Negro."14 This portrait thus appears as something of a warning from the past, like Walter Benjamin's take on Paul Klee's Angelus Novus as an angel of history; the ghostly gaze of Fanon looks back at us from a rendering of his likeness created by systems of surveillance, as something to fear.

The warning present in the Fanon image is also heralded by the parenthetical remark in its title (which appears in the titles of all

14 Franz Fanon, Black Skin, White Masks, trans. Charles Lam Markmann (London: Pluto Press, 1986), 29. 
the images in the series): Even the Dead Are Not Safe. Given that he is rendering images of historical figures, Paglen's use of this phrase seems apt. Yet this phrase also conjures up a passage from Benjamin's last major piece of writing, "Theses on the Philosophy of History," a text in which he articulates a critique of historicism and the method of Marxist historical materialism. To put it briefly here, Benjamin critiques the idea of history as a continuous path toward progress, and he instead articulates an understanding of history as a memory that arises in a moment of danger. In the same passage, he alleges historicism has been used for the benefit of the "the ruling classes." He states: "In every era the attempt must be made anew to wrest tradition away from a conformism that is about to overpower it [...] Only that historian will have the gift of fanning the spark of hope in the past who is firmly convinced that even the dead will not be safe from the enemy if he wins." ${ }^{15}$ In this passage, Benjamin describes the ways in which the past may be articulated in the present and have a way of threatening both. The representation of the figures in Paglen's eigenface images can be understood to invoke Benjamin's warning.

The work of Fanon has been referenced in connection to critiques of biometric systems before. In surveillance scholar Simone Browne's study of contemporary surveillance practices and the historical archive of transatlantic slavery, she borrows from Franz Fanon's term epidermalization in order to coin the term "digital epidermalization," which she defines as "the exercise of power cast by the disembodied gaze of certain surveillance technologies [...] that can be employed to do the work of alienating the subject by producing a truth about the racial body and one's identity (or identities) despite the subjects' claims." ${ }^{16}$ Browne's use of the term defines a denied subjectivity that is produced through the application of biometric recognition. Paglen's eigenface of Fanon visualizes this denied subjectivity with a representation of Fanon produced through the very systems of this disembodied gaze. As Paglen brings forth Fanon's figure in this piece as a ghostly warning, an invitation of di-

15 Benjamin, "Theses on the Philosophy of History," 255 (italics in original).

16 Browne, Dark Matters, 110. 
alogue between Fanon's discourse and the dialectics of recognition emerges.

Figure 31: Trevor Paglen, "Beckett" Eigenface (Even the Dead Are Not Safe), 2017. dye sublimation metal print. Image courtesy of the artist

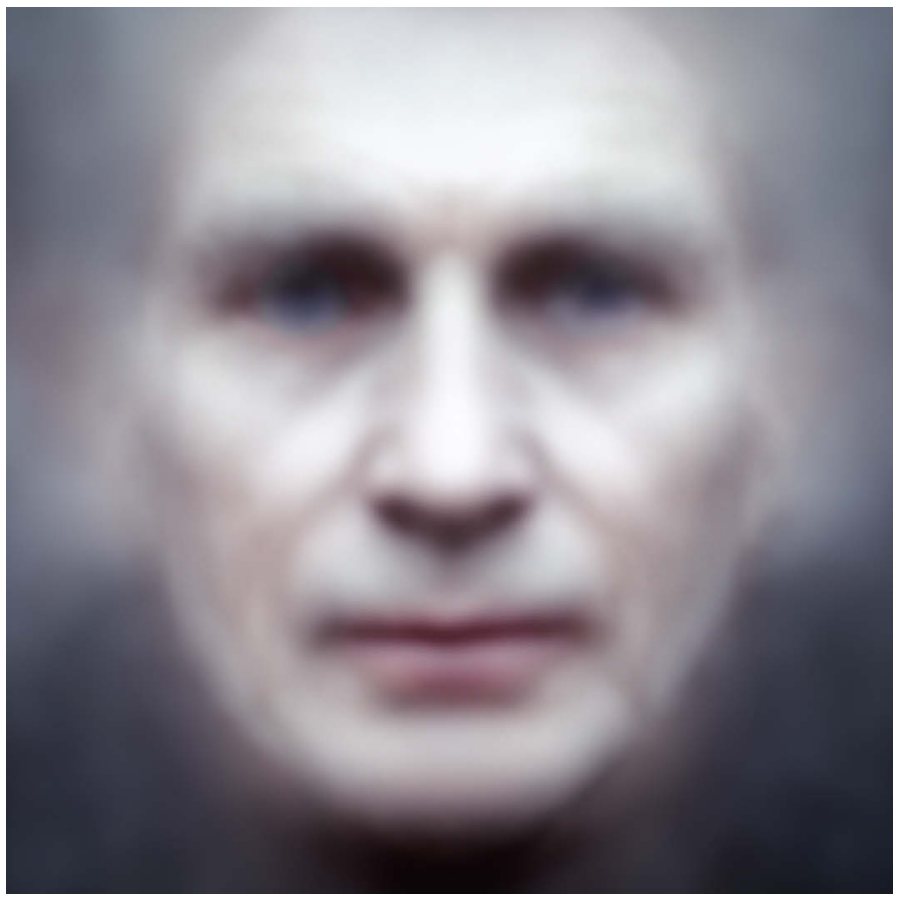

The portraits of Beckett and Weil (figure 31 and figure 32), like the Fanon portrait, present their subjects with a neutral expression and in a forward-facing pose. Fixed as an eigenface image, the head of each figure floats ominously in a sea of pixelated blur. What to make of these ghostly heads of dead revolutionaries and philosophers? As with the production of his images in the Adversarial Hallucinations series, with his eigenface portraits Paglen customizes the training set category; he chooses his subjects, all of whom stand out as archetypes of critical thinkers. Weil's face, however, wears the expression of a slight, Mona Lisa-like smile. This is perhaps because, as a woman, she has been expected to smile and to generally present 
a pleasant facial expression that is not expected of men. These are, after all, statistical averages of a collection of facial images of each figure, and as such they capture something of the public expression of each subject.

Figure 32: Trevor Paglen, "Weil" Eigenface (Even the Dead Are Not Safe), 2017. dye sublimation metal print. Image courtesy of the artist

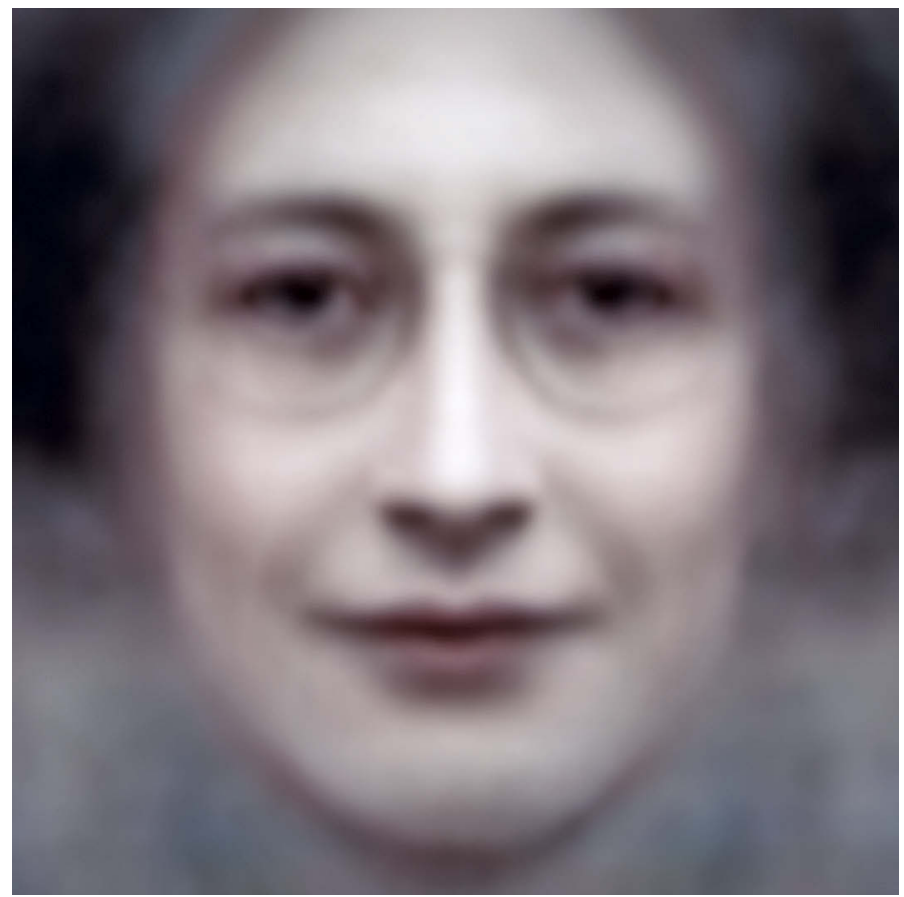

In their eigenface images, these three figures are transformed into archetypes in two ways. They are each archetypes of a particular kind of critical thinker. The work of each offers critical analyses of systems of power that speak to the contexts of facial recognition and its processes of meaning production. Beckett, for example, in his work as a novelist, playwright and poet, represents an attack on the realist tradition; he dispenses with the narrative element of a unity of time and place, instead focusing on revealing the essence of the human condition. Weil's work as a philosopher and as a polit- 
ical activist fighting for the interests of the working class included a critique of forms of oppression, whether the exploitation of capitalism or the elitist bureaucracy of Marxism. The eigenface images of each present a visual rendering of each figure as an archetype of critical thought. Yet, through the gender and race of each figure, these portraits also present archetypes of institutional categories of identification, an association I think is intentional on the artist's part. As such, we can surmise that "even the dead are not safe," that is, not safe from their inclusion within a categorical "type" as part of the identification practices of AFR technology.

In producing portraits of subjects whose work precedes their image, and whose work also contributes to a critique of the notions of power and identity, Paglen inverts a truth of recognition through automated processes. In bringing these figures "back from the dead" through the form of an eigenface classifier, he brings together the discourses of critical thought in philosophy and contemporary systems of institutional enforcement. The critiques of power that are embodied in these figures are tools. In the course of a discussion of algorithmic prediction in facial recognition, Paglen mentions: "An analysis of power, of capital, of race, [these] are the kinds of tools that you bring to the conversation that are ultimately more helpful." ${ }^{17}$ Of the eigenface portraits, he states: "someone like a Fanon or a Weil contributed to social progress precisely by breaking the law - because they were unjust laws [...] It's asking whether the development of these technologies will preclude people like Simone Weil or Franz Fanon from ever existing again." ${ }^{18}$ Paglen's question is whether, in a society that is increasingly organized through metadata and predictive systems and, as such, enforces certain kinds of "normative behavior," any revolutionary thought and action can survive. Paglen's engagement with this problem involves reference to historical figures and thereby speaks to a precedent of the technology. In other words, there is an element of answering the future

17 Charlie Robin Jones, "The Artist Trevor Paglen, The Surveillance State, and Your Life," SSENSE, May 15, 2019, https://www.ssense.com/en-us/edito $\mathrm{rial} / \mathrm{art} / \mathrm{this}$-artist-the-military-industrial-complex-and-your-life.

18 Ibid. 
(both the predictive aspect of the algorithm and a near-future society of increasing automation) with the past. Paglen intentionally exploits the "ghost-face"19 character of eigenface images as an aesthetic in these portraits, as a way of communicating how the subjects and their legacies of thought haunt the present. Paglen conjures up these figures not only as ominous reminders of the unequal power structures that underlie facial recognition technology but also as a reference to the present necessity of tools of critical discourse.

\section{Concluding Remarks}

Through both Adversarially Evolved Hallucination (2017) and Eigenface (Even the Dead Are Not Safe) (2017), Paglen gets inside some of the issues at the heart of the design of automated recognition technology. Where other scholars and artists have scrutinized the normative categories that guide facial recognition technologies, Paglen challenges how these normative categories are constructed through the technology. In appropriating the training-set phase of the image recognition algorithm, Paglen arrives at one of the ways in which the technology ascribes meaning in the world. Through his imagery he creates a space for dialogue between the dialectics of recognition and the modes of discourse in critical thinking. Together, these approaches situate the technological developments of machine vision technologies within a framework of cultural and political thought. In doing this, he also articulates an existing gap between the design and development of machine-vision systems and the cultural and political worlds in which they are deployed. His images suggest to the observer that the implications of the technology are open ended and as images, they are open to interpretation. As such, he highlights an inherent fluidity of human perception in the face of the machinic gaze.

19 Çarikçi and Özen, "A Face Recognition System," 122. 



\section{Chapter 9: Conclusion}

\section{Contribution to the field}

This study has explored the technology of automated facial recognition through the discourse of visual culture studies and contemporary artistic practice. This analysis addresses one of the central issues of contemporary visuality, that is, the technological advancement of forms of machinic vision through AFR technology and the associated shift in our understandings of what it is to see and be seen. The discussion has addressed a general problematic of automated recognition and framed its enactment as a technical, cultural and philosophical process. A primary theoretical aim has been to approach the recognition process of AFR technology as an example of machinic vision, that is, a mode of perception involving a disembodied perspective, and to analyze how it operates in contemporary society. Taking the processes of an AFR method as its point of departure, this study has shown how machinic vision is technically defined through an automated operation of recognition. It has, further, explored a history in which statistics and vision have been intimately connected, revealing this connection to be an organizing principle of AFR processes and a representational mechanism through which automated recognition occurs. Contemporary examples of artistic practice have provided further insight into the contexts of AFR implementation and suggested new approaches to the conceptualization of this technology. As I have shown, the artworks included in this study also actively reimagine the processes placing them in dialogue with cultural and socio-political contexts 
broadening our understanding of the dialectics of recognition in a machine-vision process.

Through its exploration of the shifting role of the image from pictorial to algorithmic with the advent of digital networks, this analysis contributes to the field of visual culture studies. ${ }^{1}$ Technologists have argued that machines do not see - that AFR technology does not relate to historical discourses on visuality or photography but rather introduces an entirely new digital landscape in which all visual input, whether pictorial or coded, is and should be considered as data. While taking this view into account, I argue that contemporary digital technologies and the infrastructures in which they operate do not present an entirely new world but rather carry within them historical continuities of cultural and visual logic. With reference to concepts such as the technical image and the operational image, ${ }^{2}$ notions that track this shift in the role of images as information, I have approached the eigenface image as an object of inquiry. The eigenface image provides an image of data and, as such, serves as a meeting point for human perception and algorithmic perception. I have used the example of eigenface to reflect on this tension between image and data. This analysis does not confound images with data but rather attempts to clarify the complex relationship between the two. In relating the representational mechanisms of AFR technology to the historical practice of composite portraiture within the sciences, philosophy and art, I have explained the way this technology relies on a cultural logic that involves the merging of statistics with vision. By tracing this statistical way of seeing, I have brought to light the nuanced relationship between images and data, rather than simply suggesting that there is a mutually exclusive relationship between these two categories.

This analysis also makes a methodological contribution in its departure from traditional approaches within the field of visual

1 For more on this topic see Hito Steyerl, "A Sea of Data: Apophenia and Pattern (Mis-)Recognition," e-flux Journal 72 (April 5, 2016), https://www.e-f Zlux.com/journal/72/60480/a-sea-of-data-apophenia-and-pattern-mis-re cognition/.

2 Vilem Flusser, Into the Universe, 10; Farocki, War at a Distance. 
culture: namely, it expands the scope of analysis beyond the image to include the processes by which the image is produced. In the context of analyzing machinic vision, this is imperative if we are to understand the ways in which the image operates and to reveal, interrogate and critique the conditions of algorithmic vision. Referring to the work of Jonathan Crary ${ }^{3}$ and his analysis of the historical construction of vision through the position of the observer, this study of a particular AFR method has attempted to explain how its technology embodies a machinic observer, and to explore the historical continuities and discontinuities present in this new kind of visual organization. This study has shown how an analysis of the visuality of AFR technology can contribute to a broader understanding of the varying modes of perception found in contemporary society and their roles in intervening across social, political and cultural terrains.

This analysis joins certain other scholarly works in critiquing the limitations of AFR technology from a perspective in the humanities. ${ }^{4}$ Drawing on theoretical perspectives from surveillance studies, gender and race studies, and media and communications studies, these previous scholarly works have not approached the subject matter from the standpoint of the sciences, that is, from within the fields in which these technologies have been developed, but from beyond them, in order to achieve more expansive notions about what counts as the success or failure of biometric technologies and to understand their cultural, political and social implications. Similarly, this analysis approaches AFR technology as a form of machinic vision by bringing its processes into a dialogue with a theoretical framework premised on visual culture and its associated political, cultural and philosophical ideas.

The findings of this analysis support a central critique leveled by the authors of many of these previous works: that recognition by an AFR system enforces normative categories of institutionally based identity in the service of ever-tighter biopolitical control. This is analyzed through the discussion on the ways of seeing em-

3 Crary, Techniques of the Observer.

4 See discussion in Cultural Analysis of Biometrics: Previous Scholarship, p. 22. 
bedded in the eigenface method and its reductive representational mechanisms. In an operation of recognition carried out by an AFR system, any understanding of identity that is both subjective and fluid gets negated. At a time when identity politics is in the ascendant and self-generated notions of identity are crucial to political agency, AFR systems automate the enforcement of institutionally based identities. This enforcement of normative categories of identity has the potential to disproportionately affect those groups that are already vulnerable and marginalized in relation to systems of governance including the distribution of state services, border control and policing - three key contexts in which AFR is increasingly being employed. For AFR systems are not only implemented in contexts of security and risk mitigation; they are also used to grant or deny access to resources. The negation of recognition may be just as dangerous as being unwittingly registered in a biometric database. This study of the machinic ways of seeing used in a particular AFR method of eigenface has revealed a history of the enforcement of institutionally based identities; these identities are further operationalized through these automated technologies of recognition.

\section{Revisiting the Aim: Looking Back}

In sum, the problem with AFR and its machinic way of seeing is that it applies a statistical and thereby reductive method of recognition to a subject whose identity cannot ultimately be measured, defined or known by these means. This study began with three research questions, the first of which asked how recognition is defined in an AFR method. The analysis of the eigenface algorithm in Part I explored three aspects that constitute recognition and perception by algorithm: the statistical pattern recognition method of Principal Component Analysis (PCA), the production of facial aggregates called eigenvectors and the formation of the subspace called the face space. The eigenface method recognizes individual faces by differentiating them from a statistical average. The primary representational mechanism of eigenface, that is, the algorithm's means of coming to know and recognize faces, relies on a statistical meth- 
od. Recognition through the eigenface method occurs through the merging of statistics with vision. This merging of statistics with vision as a means of recognition has various ontological implications. One of these was explored in this thesis in the discussion of the historical antecedent of the eigenface method: Galton's composite portraiture and his method of using statistical representations of faces to uncover sociological types. The discussion of Galton's approach to composite portraiture responded to the second research question of this study, on the historical continuities and discontinuities between AFR methods and their antecedents. With this account of Galton's composite-portrait practice, this study emphasized the cultural and historical context of the origins of a merging of statistics and vision, namely the way Galton's broader project of eugenics and the visualization of reductive, normative sociological categories inform a particular statistical way of seeing.

An alternative to this statistical way of seeing - and of interpreting the significance of the composite portrait - is provided by Ludwig Wittgenstein, who puts forward an alternative take on the meaning of the composite's aesthetic form. Approaching the composite portrait as an object of study within the field of philosophy and within his project of investigating language games, Wittgenstein argues that we should recognize that the composite portrait contains multiple overlapping forms. Rather than perceiving the composite as an expression of statistical reduction, he sees it as an expression of a perceptual fluidity. Through his concept of aspect perception, Wittgenstein describes a perceptual ability to perceive forms shifting into other forms. I argue that, in his approach to the composite portrait, Wittgenstein provides an inherently utopian vision that expresses the kind of fluidity found in current discourses of identity politics and the politics of representation, a vision that resists the reductive processes of recognition through AFR systems and their utilization of biopolitical control.

Wittgenstein's alternative take on the composite portrait was expanded upon in Part II, which explored the artistic engagements of contemporary artists with the technology of facial recognition. This brings us to the third and final research question of this study, which asked how contemporary artists have articulated and prob- 
lematized the cultural implications of AFR technology. I discussed examples of contemporary artistic practice in order to provide further insight into the contexts of AFR implementation and suggest new ways of conceiving of this technology. Each artist presents an engagement with facial recognition technology within a particular cultural discourse, thus answering a central question posed by this technology: what meaning can be derived through a process of automated facial recognition?

\section{The Composite Form}

Throughout this study, the motif of the composite portrait has appeared in different forms. The composite emerges as an aesthetic form that is expressive of how information is structured. This investigation has shown that the composite form, as it has expressed itself in practices of facial recognition, has two diverging and contradictory meanings that are developed through two different discourses. In the analysis of eigenface, the digital composite was introduced as the phantom face that peers out from the eigenface image, as the visualization of a statistically constructed pattern of multiple faces. In Francis Galton's work, the composite portrait was deployed in the attempt to produce images of sociological types. Although ultimately failing in this endeavor, Galton's composite portraits were an original attempt to give visibility to a knowledge only possible and operational through statistics and to make this knowledge accessible to the human eye. The eigenface image and Galton's composite portrait are examples of the composite form being utilized within the areas of science and governance and operationalized as a predictive tool through reductive logic. But in Wittgenstein's perceptual approach to the composite portrait - now transplanted to the discourse of philosophy - it is an expression of multiplicity in which all the phenomena that make up the composite lie open to view. ${ }^{5}$ The composite portrait is an expression of a relationship between varying forms. The possibilities of meaning

5 Wittgenstein, Philosophical Investigations, 126. 
contained within the perception of the composite form also, on this view, multiply. This study concludes that the composite form can be expressive of an entirely different and contrary meaning from the one suggested by the reductive, statistical interpretation of the form.

This alternative approach to the composite form was further explored in Part II. Through a discussion of works by three artists, Thomas Ruff, Zach Blas and Trevor Paglen, I examined the varied expressions of the composite motif in contemporary artworks engaging with the theme of facial recognition. The artworks in this study extract the composite and the structures of AFR knowledge from the discourses of science and governance, and they explore the myriad representational uses of the composite. In Ruff's series of andere Portraits, silk-screened photographic composite mugshots explore the notion that the act of representation itself constructs an identity. The binary and analogue composite form used in this series of portraits is such that one's eyes are never able to rest on a single face. Like a kind of large-scale visualization of Wittgenstein's concept of aspect perception, these portraits express a constant fluidity - between faces and, also, between notions of gender. Blas's sculptural masks, in his Facial Weaponization Suite, transform composite portraiture into physical form. Blas's sculptural composites reference a notion of excess: that is, the multiplicity of facial forms that constitute his masks counter and resist the singularity of a recognition operation by an AFR system. A key contribution of Blas's work is his use of the form of the composite, that is, the aesthetic form that structures the information used by an AFR system, to counter the very operation of this technology. Drawing on discourses in post-colonial theory and theories of political agency, Blas uses his masks to reassert an unruliness in representations of the body. His work also points towards a notion of the subject as composite, through a political strategy of collectivism. Paglen's use of the composite form in two series, Adversarial Hallucinations and Eigenface (Even the Dead Are Not Safe), appropriates the categories of knowledge used to train AI systems of recognition. By presenting algorithmic-based imagery, his composites reveal a relationship between training data and an algorithmic ability not only to recognize 
but to reimagine physical form. Paglen's use of literary metaphors and ideological concepts to categorize training data speaks to the deeper truths that structure contemporary surveillance systems and offers a means of critiquing their cultural logic.

Through these alternative explorations of the composite form and facial recognition, these artworks reassert a visual field that is fluid, open, mutable and dynamic. As such, they are able to reveal the limitations of a machinic way of seeing and instead put forward a way of seeing that can measure up to the truth of the recognized subject. As Jenny Edkins states, "the contemporary face - the face of biopolitics and surveillance - forces a being into presence, but into presence as an object not a person. The being, in all its mystery, its unknowability, is missing." As well as providing these expressions of an open, mutable perception, these artworks allow for a measure of unknowability in relation to the subject. This space allows a subject to choose her own identity or identities, based on an embodied, subjective experience, and also allows the subject to have the right to reject enforced, institutional categories of identity. This right continues in its importance because institutional identities are constructed by institutions of governance - the same institutions of governance that have historically deemed black and brown people sub-human, cast Jews as the enemy and today detain a growing population of migrants in detention camps, defining these groups as stateless and thereby without access to certain, inalienable rights.

\section{Recommendations: Looking Forward}

During the final stages of writing this book, the New York Times carried a news report about the first known use of AFR by a government for the purposes of racial profiling. ${ }^{7}$ The report described the use of AI by authorities in China, in particular, the use of facial

6 Edkins, Face Politics, 7.

7 Paul Mozur, "One Month, 500,000 Face Scans: How China Is Using AI to Profile a Minority," The New York Times, April 14, 2019. https://www.nytimes. 
recognition technology to surveil and track members of the Uighur community, a minority ethnic Muslim population, for the purposes of law enforcement. ${ }^{8}$ The report explains that recognition is strictly based on appearance, as Uighurs look distinct from the majority Han population in China, which makes it "easier for software to single them out." The report describes the creation by Chinese authorities of face-image databases not only for Uighurs but also "for people with criminal records, mental illnesses, records of drug use and those who petitioned the government over grievances. ${ }^{.10}$ In light of the historical analysis offered in this study, we may say that, with the use of AFR technology in China, history is repeating itself. My analysis of the facial representation mechanisms found within the eigenface algorithm and the practice of differentiating facial groups from a "norm" makes it clear that the possibility of using this technology for racial profiling purposes is built into the very ways of seeing that underlie AFR processes. The report on the use of AFR by the Chinese authorities also describes a shift in the development of AI technologies; where before democratic societies had the upper hand in this sector, authoritarian regimes are now producing a new generation of tech startups that cater to their needs. ${ }^{11}$ This authoritarian use of AFR processes not only reflects one possible path of future development for this technology but also recalls a history in the discourses and practices of the politics of representation. In conclusion, this analysis recommends looking toward the aesthetic forms that structure algorithmic knowledge as sources of inquiry and contestation. In doing so, it has engaged with a historical discourse on the relationship between visual perception and knowledge, and it has sought to relate this to an alternative way in which technology might define and organize visual perception in the future.

com/2019/04/14/technology/china-surveillance-artificial-intelligence-raci al-profiling.html?partner=IFTTT.

8 Ibid.

9 Ibid.

10 Ibid.

11 Ibid. 
This book began with a description of an experience I had of being biometrically registered at an airport border crossing, and it ends with this report of the first known use of AFR for racial profiling purposes. The growing use of AFR in a variety of areas, from risk mitigation and the control of people's movements and access to resources to the profiling of vulnerable groups, indicates an intensification of the interconnections between its automated processes and contemporary notions of recognition, representation and identity. It also indicates the necessity of approaching algorithmic processes as possible objects of critique, of problematizing their logic and dissecting and seeing through their everyday use. This means ensuring that the processes and narratives of algorithms - their weaknesses, their logic and their implications - are transparent to us. This analysis joins those of other scholars in the field of the humanities who have found it necessary, and possible, to question the logic of algorithms and to dissect their use. The issues that arise from the technological development of AI and the application of AFR reveal that contemporary shifts in technology go hand in hand with a shift in culture. In the face of our increasing dependence on AFR technology, this visual culture analysis of the phenomenon has, in sum, amounted to an academic version of an A.I model of always keeping the "human in the loop" - by keeping the humanities in the loop and recognizing that they are, almost always, already in the loop. 


\section{References}

Adler, Dan. "The Apparatus: On the Photography of Thomas Ruff." Art Journal 75, no. 2 (Summer 2016): 66-87.

Ajana, Btihaj. Governing through Biometrics: The Biopolitics of Identity. Basingstoke: Palgrave Macmillan, 2013.

Amaria, Kainaz. "The 'Girl in the Blue Bra." NPR, December 21, 2011. https://www.npr.org/sections/pictureshow/2011/12/21/1440983 84/the-girl-in-the-blue-bra.

Amoore, Louise. "Biometric Borders: Governing Mobilities in the War on Terror." Political Geography 25, no.3 (March 2006): 336-51. https://doi.org/10.1016/j.polgeo.2006.02.001.

Ballantyne, Michael, Robert S. Boyer, and Larry Hines. "Woody Bledsoe: His Life and Legacy.” AI Magazine 17, no. I (Spring 1996): 7-20. https://doi.org/10.1609/aimag.v17i1.1207.

Benjamin, Walter. "Theses on the Philosophy of History." In Walter Benjamin, Illuminations, translated by Harry Zohn, 253-264. New York: Schocken Books, 2007.

Berger, John. Ways of Seeing. London: Penguin Books, 1972.

Blas, Zach. Facial Weaponization Communiqué: Fag Face (2012). Video. https://vimeo.com/57882032.

-. "Informatic Opacity: Biometric Facial Recognition and the Aesthetics and Politics of Defacement." PhD dissertation, Duke University, 2014.

-. "A Cage of Information, or, What is a Biometric Diagram?" In Documentary across Disciplines, edited by Erika Balsom and Hila Peleg, 80-90. Cambridge, MA: MIT Press, 2016.

—. "Fag Face." Recaps Magazine. Accessed May 3, 2019. http://recaps magazine.com/review/fag-face-by-zach-blas/. 
Bledsoe, Woodrow Wilson. "The Model Method in Facial Recognition." Technical Report PRI 15. Palo Alto, CA: Panoramic Research, Inc., 1964.

Bridle, James. "Drone," James Bridle website. Accessed June 3, 2019. https://jamesbridle.com/works/category:drone.

Broomberg, Adam, and Oliver Chanarin. "The Bone Cannot Lie." In Adam Broomberg and Oliver Chanarin, Spirit is a Bone, 206-238. London: Mack, 2015.

Browne, Simone. Dark Matters: On the Surveillance of Blackness. Durham, NC: Duke University Press, 2015.

Burson, Nancy. First and Second Beauty Composites (1982). Gelatin silver prints from computer generated negatives.

-. Warhead I (Reagan 55\%, Brezhnev 45\%, Thatcher less than 1\%, Mitterand less than 1\%, Deng less than 1\%) (1982). Gelatin silver prints from computer generated negatives.

Çarikçi, Müge, and Figen Özen. "A Face Recognition System Based on Eigenfaces Method." Procedia Technology 1 (2012): 118-23. https://doi.org/10.1016/j.protcy.2012.02.023.

"Chaplin Modern Times - Factory Scene (late afternoon)." YouTube video, 4:13. Posted by "Olaf V/s Minions," September 5, 2015. https:/www.youtube.com/watch?v=HPSK4zZtzLI.

Cirio, Paolo. Obscurity (2016). Inkjet prints. https://paolocirio.net/ work/obscurity/.

Cole, Teju. "A True Picture of Black Skin." The New York Times Magazine, February 18, 2015. https:/www.nytimes.com/2015/02/22/ magazine/a-true-picture-of-black-skin.html.

Crary, Jonathan. Techniques of the Observer: On Vision and Modernity in the Nineteenth Century. Cambridge, MA: MIT Press, 1990.

Daston, Lorraine, and Peter Galison. Objectivity. Cambridge: Zone Books, 2007.

Dewey-Hagborg, Heather. Stranger Visions (2012-2013). Sculpture.

Dick, Philip K. A Scanner Darkly. New York: Vintage Books, 1991.

Dorment, Richard. "Photography in Focus." The Telegraph, May 29, 2003. http://www.telegraph.co.uk/culture/3595514/PHOTOGRA PHY-IN-FOCUS-The-deadpan-images-created-by-ThomasRuff-of-nameless-individuals-and-equally-anonymous-places- 
are-masterpieces-of-austere-neutrality.-By-Richard-DormentNow-for-something-completely-indifferent.html.

Drück, Patricia. Das Bild des Menschen in der Fotografie: Die Porträts von Thomas Ruff. Dietrich Reimer, 2004.

Edkins, Jenny. Face Politics. London: Routledge, 2015.

Elahi, Hasan. Hasan Elahi website. Accessed June 3, 2019. http:// elahi.umd.edu.

Ellis, Havelock. The Criminal, New York: Scribner \& Welford, 1890.

Enwezor, Okwui. "The Conditions of Spectrality and Spectatorship in Thomas Ruff's Photographs." In Thomas Ruff: Works 1979-2011, 9-19. München: Schirmer Mosel, 2012.

Fanon, Franz. Black Skin, White Masks. Translated by Charles Lam Markmann. London: Pluto Press, 1986.

Farocki, Harun. War at a Distance. Video. Berlin: Harun Farocki Filmproduktion, ZDF, 2003.

—. "Phantom Images." Translated by Brian Poole. Public 29 (Spring 2004): 12-22.

Fast, Omer. 5,000 Feet is the Best (2011). Digital video. http://www. gbagency.fr/en/42/Omer-Fast/\#!/5-000-Feet-is-the-Best/site_ video_listes/88

Feldman, Alexandra. "Face Recognition: Final Project CS 129, Spring 2011." Computer Science at Brown University. Accessed April 25, 2015. http://cs.brown.edu/courses/csci1290/2011/results/final/ amfi/.

Flusser, Vilem. Into the Universe of Technical Images. Translated by Nancy Ann Roth. Minneapolis, MN: University of Minnesota Press, 2011.

Forensic Architecture. Drone Strike in Mir Ali (2013). Digital video. https://forensic-architecture.org/investigation/drone-strikein-mir-ali.

Foucault, Michel, "The Subject and Power," Critical Inquiry 8, no. 4 (Summer, 1982): 777-795.

Foucault, Michel. Discipline and Punish. London, Penguin Books, 1991.

Galton, Francis. Inquiries into Human Faculty and Its Development. 2nd ed. Reprint of the 1907 London: Dent edition, galton.org, 2018. 
http://galton.org/books/human-faculty/SecondEdition/text/ web/human-faculty4.htm.

Galton, Francis. "Composite Portraits," Popular Science Monthly 13 (August 1878).

Gates, Kelly A. Our Biometric Future: Facial Recognition Technology and the Culture of Surveillance. New York: New York University Press, 2011.

Han, Bang Geul. Referential Gaze (2015). Inkjet prints.

Hoel, Aud Sissel. "Operative Images: Inroads to a New Paradigm of Media Theory." In Image - Action - Space: Situating the Screen in Visual Practice, edited by Luisa Feiersinger, Kathrin Friedrich, and Moritz Queisner, 11-27. Berlin: DeGruyter, 2018.

Hoel, Aud Sissel, and Frank Lindseth. "Differential Interventions: Images as Operative Tools." Photomediations: A Reader, edited by Kamila Kuc and Joanna Zylinska, 177-183. London: Open $\mathrm{Hu}-$ manities Press, 2016.

Introna, Lucas, and David Wood. "Picturing Algorithmic Surveillance: The Politics of Facial Recognition Systems." Surveillance and Society 2, no. 2/3 (2004): 177-198. https://doi.org/10.24908/ ss.v2i2/3.3373.

Johnston, John. “Machinic Vision.” Critical Inquiry 26, no. 1 (Autumn 1999): 27-48, https://doi.org/10.1086/448951.

Jones, Charlie Robin. "TheArtist Trevor Paglen, The Surveillance State, and Your Life." SSENSE, May 15, 2019. https://www.ssense.com/ en-us/editorial/art/this-artist-the-military-industrial-complex -and-your-life.

Kedmey, Karen. "Hasan Elahivs. The FBI:The Art ofSelf-Surveillance.” Artsy, May 27, 2016. https://www.artsy.net/article/artsy-hasanelahi-vs-the-fbi-the-art-of-self-surveillance.

Kemp, Cornelia. "The Other Face." The Other Face: Metamorphoses of the Photographic Portrait, edited by Cornelia Kemp and Susanne Witzgall, 8-13. Munich: Prestel Verlag, 2002.

Kun, Jeremy. "Eigenfaces, for Facial Recognition." Math Programming (blog), July 27, 2011. https://jeremykun.com/2011/07/27/eigen faces/.

Lee, Wonju, Minkyu Cheon, Chang-ho Hyun, and Mignon Park. "Best Basis Selection Method Using Learning Weights for Face 
Recognition." Sensors (Basel) 13, no. 10 (October 2013): 12830-51. https://doi.org/10.3390/s131012830.

Levitt, Helen. Manhattan Transit: The Subway Photographs of Helen Levitt. Köln: Walther König, 2017.

Lyon, David. "Facing the Future: Seeking Ethics for Everyday Surveillance." Ethics and Information Technology 3, no. 3 (2001): 171180. https://doi.org/10.1023/A:1012227629496.

-. "Surveillance as Social Sorting: Computer Codes and Mobile Bodies." In Surveillance as Social Sorting: Privacy, Risk and Digital Discrimination, edited by David Lyon, 13-30. London: Routledge, 2003.

Magnet, Shoshana. When Biometrics Fail: Gender, Race and the Technology of Identity. Durham, NC: Duke University Press, 2011.

Marx, Karl. Capital: A Critique of Political Economy. Vol. 1. Translated by Samuel Moore and Edward Aveling. Moscow: Progress Publishers, 1887.

McNally, David. Monsters of the Market: Zombies, Vampires, Global Capitalism. Chicago, IL: Haymarket Books, 2012.

Miłkowski, Marcin. Explaining the Computational Mind. Cambridge, MA: MIT Press, 2013.

Mitchell, W. J. T. Iconology. Chicago, IL: The University of Chicago Press, 1986.

Mitscher, Clemens. "1987 - Opfer." Clemens Mitscher website. Accessed May 25, 2017. http://mitscher.de/content/?page_id=265.

Mozur, Paul. "One Month, 500,000 Face Scans: How China Is Using AI to Profile a Minority." The New York Times, April 14, 2019. https://www.nytimes.com/2019/04/14/technology/china-surveil lance-artificial-intelligence-racial-profiling.html?partner=IFT TT.

Mumford, Lewis. Pentagon of Power: The Myth of the Machine, Vol. II, New York: Harcour Brace Jovanovich, 1974.

Nisbet, Robert A. "Social Science." Encyclopedia Britannica, September 27, 2018. https://www.britannica.com/topic/social-science/ The-2oth-century.

Paglen, Trevor. I Could Tell You But Then You Would Have to Be Destroyed By Me: Emblems From the Pentagon's Black World. Brooklyn: Melville House, 2010. 
-. "Invisible Images (Your Pictures Are looking At You)." The New Inquiry, December 8, 2016. https://thenewinquiry.com/invisi ble-images-your-pictures-are-looking-at-you/.

-. Sight Machine. Performance. January 14, 2017, San Francisco, CA.

-. A Study of Invisible Images. Exhibition. September 8-October 21, 2017, Metro Pictures Gallery, New York, NY.

-. "Machine Realism." In I Was Raised on the Internet, edited by Omar Kholeif, 112-118. Munich: Prestel, 2018.

Paré, Anthony. "Rhetorical Genre Theory and Academic Literacy." Journal of Academic Language \& Learning 8, no. I (2014): A83-A94.

Pearson, Karl. "LIII. On Lines and Planes of Closest Fit to Systems of Points in Space." The London, Edinburgh, and Dublin Philosophical Magazine and Journal of Science 2, no. 11 (1901): 559-72.

Pearson, Karl. The Life, Letters and Labours of Francis Galton Vol 2. Cambridge at the University Press, 1924.

Peters, John Durham. "Information: Notes Toward a Critical History." Journal of Communication Inquiry 12, no. 2 (1988): 9-23. https:// doi.org/10.1177/019685998801200202.

Ploeg, Irma van der. "Biometrics and the Body as Information: Normative Issues of the Socio-technical Coding of the Body." In Surveillance as Social Sorting: Privacy, Risk and Digital Discrimination, edited by David Lyon, 57-73. London: Routledge, 2003.

Quetelet, Adolphe. Lettres sur la théorie des probabilities appliquée aux sciences marales et politiques. Bruxelles: M. Hayez, 1846.

Robbins, Christopher. The Invisible Air Force: The Story of the CIA's Secret Airline. London: Macmillan, 1981.

Ruff, Thomas. Andere Porträts +3D. Germany: Cantz Verlag, 1995.

-. e-mail interview, 15 May 2016

Scheib, Vincent. "Eigenfaces of UNC." Accessed April 25, 2015. http://www.scheib.net/school/uncfaces/index.html.

Schivelbusch, Wolfgang. The Railway Journey: The Industrialization of Time and Space in the 19th Century. Berkeley, CA: The University of California Press, 1986.

Schultze-Naumburg, Paul. Kunst und Rasse, Munich: J.F. Lehmanns Verlag, 1928.

Sekula, Allan. "The Body and the Archive." October 39 (Winter 1986): 3-64. 
Sirovich, Lawrence, and Michael Kirby. "Low-dimensional Procedure for the Characterization of Human Faces." Journal of the Optical Society of America 4, no. 3 (April 1987): 519-24.

Stephens, Elizabeth. "Francis Galton's Composite Portraits: The Productive Failure of a Scientific Experiment." Unpublished manuscript. June 2013. https://www.researchgate.net/publication/ 323275029_Francis_Galton's_Composite_Portraits_The_Produc tive_Failure_of_a_Scientific_Experiment.

Steyerl, Hito. How Not to Be Seen:A Fucking Didactic Educational.MOV file (2013). Digital video. https://www.artforum.com/video/ hito-steyerl-how-not-to-be-seen-a-fucking-didactic-educatio nal-mov-file-2013-51651.

-. "A Sea of Data: Apophenia and Pattern (Mis-)Recognition." e-flux Journal 72 (April 5, 2016). https://www.e-flux.com/jour nal/72/60480/a-sea-of-data-apophenia-and-pattern-mis-recog nition/.

Turk, Matthew. "Over Twenty Years of Eigenface." ACM Transactions on Multimedia Computing Communications and Applications 9, no. 1 (October 2013): 1-5. http://dx.doi.org/10.1145/2490824.

Turk, Matthew, and Alex Pentland. "Eigenfaces for Recognition." Journal of Cognitive Neuroscience 3, no. 1 (Winter 1991): 71-86.

Valentine, Ben. "Weaponizing Our Faces: An Interview with Zach Blas.” Vice,July 10, 2014.https://www.vice.com/en_us/article/vdp zaa/weaponizing-our-faces-an-interview-with-zach-blas-715.

Valentine, Tim, Michael B. Lewis, and Peter J. Hills. "Face-Space: A Unifying Concept in Face Recognition Research." Quarterly Journal of Experimental Psychology 69, no. 10 (2016). 1996-2019. https:// doi.org/10.1080/17470218.2014.990392.

Van Gelder, Hilde, and Helen W. Westgeest. Photography Theory in Historical Perspective. Hoboken: Wiley-Blackwell, 2011.

Virilio, Paul. The Vision Machine. Translated by Julie Rose. Bloomington, IN: Indiana University Press, 1994.

Wayman, James L. "The Scientific Development of Biometrics over the Last 40 Years." In The History of Information Security: A Comprehensive Handbook, edited by Karl de Leeuw and Jan Bergstra, 263-74. Amsterdam: Elsevier, 2007. 
Wittgenstein, Ludwig. Preliminary Studies for the "Philosophical Investigations": Generally Known as The Blue and Brown Books. Oxford: Basil Blackwell, 1958.

-. Philosophical Investigations. Translated by G. E. M. Anscombe, P. M. S. Hacker and J. Schulte. 4th ed. Chichester: Blackwell, 2009. Wolthers, Louise. "Introduction: Watching Europe and Beyond Surveillance, Art and Photography in the New Millennium." In Watched! Surveillance, Art and Photography, 8-23. Köln: Walther König, 2016.

Woodward, John D. Jr.. Biometrics: Facing Up To Terrorism. Santa Monica, CA: Rand Corporation, 2001. https://www.rand.org/ pubs/issue_papers/IP218.html.

$\emptyset$ rum, Kristoffer. Ambivalent Physiognomy. University of Copenhagen, May 22, 2017. Performance. 


\section{List of Images}

Fig. 1: Woodrow W. Bledsoe, "Examples of photograph pairs used in the study," 1964. The Model Method of Facial Recognition, Palo Alto: Panoramic Research, Inc.

Fig. 2: Woodrow W. Bledsoe, "Double exposure shows that the two different subjects are surprisingly similar on a point by point basis," 1964. The Model Method of Facial Recognition, Palo Alto: Panoramic Research, Inc.

Fig. 3: Woodrow W. Bledsoe, "Double exposure shows that the two poses of the same subject have very little in common when considered on a point by point basis," 1964. The Model Method of Facial Recognition, Palo Alto: Panoramic Research, Inc.

Fig. 4: Lawrence Sirovich and Michael Kirby, "Sample face on top and its caricature below it," in "Low-dimensional Procedure for the Characterization of Human Faces," Journal of the Optical Society of America 4, no. 3 (April 1987): 519-24.

Fig. 5: Eigenfaces of faces from the ORL face database, 2004. () AT\&T Laboratories, Cambridge, UK.

Fig. 6: Lawrence Sirovich and Michael Kirby, "Average face based on an ensemble of 115 faces." in "Low-dimensional Procedure for the Characterization of Human Faces," Journal of the Optical Society of America 4, no. 3 (April 1987): 519-24. 
Fig. 7: Vincent Scheib, "Eigenfaces of UNC," http://www.scheib. net/school/uncfaces/index.html.

Fig. 8: Alexandra Feldman, "Face Recognition: Final Project CS 129, Spring 2011," Computer Science at Brown University, http:// cs.brown.edu/courses/csci1290/2011/results/final/amfi/.

Fig. 9: Wonju Lee, Minkyu Cheon, Chang-ho Hyun, and Mignon Park, "Best Basis Selection Method Using Learning Weights for Face Recognition," Sensors (Basel) 13, no. 10 (October 2013): 12830-51, https://doi.org/10.3390/s131012830.

Fig. 10: Francis Galton, "Composites assembled for photography" in Popular Science Monthly Volume 13, (August 1878).

Fig. 11: Francis Galton, "Step one in assembling a composite photograph" in Popular Science Monthly Volume 13, (August 1878).

Fig. 12: Adolphe Quetelet, "Binomial distribution, 999 trials, histogram," in Lettres sur la theorie des probabilites appliquée aux sciences (Bruxelles, M. Hayez, 1846): 103.

Fig. 13: Francis Galton, Composite Portraiture. Illustration Plates in "Inquiries into Human Faculty and Its Development", 1883, p. 7.

Fig. 14: Francis Galton, "Indian Portraits of Alexander the Great with Composite in centre" in Karl Pearson, The Life, Letters and Labours of Francis Galton, Vol 2, 1924: 296.

Fig. 15: Francis Galton, Frontispiece for Havelock Ellis, The Criminal, (New York: Scribner \& Welford, 1890).

Fig. 16: The components of the composite photo: Wittgenstein's sisters Gretl, Helene and Hermine, and Ludwig. CLudwig Wittgenstein Trust, Cambridge. 
Fig. 17: Ludwig Wittgenstein's composite portrait, 1928. Photographs by Moritz Nähr. CLudwig Wittgenstein Trust, Cambridge.

Fig. 18: Thomas Ruff, andere Portrait, Nr. 71/65 (1994/95). Image courtesy of the artist.

Fig. 19: Thomas Ruff, andere Portrait, Nr. 102/13, 1994-95. Image courtesy of the artist.

Fig. 20: Thomas Ruff, andere Portrait, Nr. 102/125, 1994-95. Images courtesy of the artist.

Fig. 21: Thomas Ruff, Venice Biennale, 1995. Images courtesy of the artist.

Fig. 22: Egyptian army soldiers arrest a female protester during clashes at Tahrir Square in Cairo on Dec. 17. (c) Stringer/ Reuters/Landov.

Fig. 23: Zach Blas, Mask - November 20, 2013, New York, NY, Facial Weaponization Suite, Photo by Christopher O'Leary. Image courtesy of the artist.

Fig. 24: Zach Blas, Fag Face, Facial Weaponization Communiqué: Fag Face, video still (2012) Image courtesy of the artist.

Fig. 25: Zach Blas, Fag Face Mask - October 20, 2012, Los Angeles, CA. Facial Weaponization Suite. Photo by Christopher O'Leary. Image courtesy of the artist.

Fig. 26: Zach Blas, Facial Weaponization Suite: Procession of Biometric Sorrows, Museo Universitario Arte Contemporáneo (MUAC) Mexico City, Mexico (5 June 2014). Photo by Orestes Montero Cruz. Image courtesy of the artist. 
Fig. 27: Trevor Paglen, "Highway of Death (Corpus: The Aftermath of the First Smart War)" Adversarially Evolved Hallucination, 2017. dye sublimation metal print. Image courtesy of the artist.

Fig. 28: Trevor Paglen, "Vampire (Corpus: Monsters of Capitalism)". Adversarially Evolved Hallucination, 2017. dye sublimation metal print. Image courtesy of the artist.

Fig. 29: Trevor Paglen, training set images for "Vampire (Corpus: Monsters of Capitalism)" Adversarially Evolved Hallucination, 2017. Image courtesy of the artist.

Fig. 30: Trevor Paglen "Fanon" Eigenface (Even the Dead Are Not Safe), 2017. dye sublimation metal print. Image courtesy of the artist.

Fig. 31: Trevor Paglen, "Beckett" Eigenface (Even the Dead Are Not Safe), 2017. dye sublimation metal print. Image courtesy of the artist.

Fig. 32: Trevor Paglen, "Weil" Eigenface (Even the Dead Are Not Safe), 2017. dye sublimation metal print. Image courtesy of the artist. 